\title{
Sources of Low-Energy Events in Low-Threshold Dark-Matter and Neutrino Detectors
}

\author{
Peizhi Du $\odot,{ }^{1}$ Daniel Egana-Ugrinovic, ${ }^{2}$ Rouven Essig, ${ }^{1}$ and Mukul Sholapurkar ${ }^{1}$ \\ ${ }^{1}$ C. N. Yang Institute for Theoretical Physics, Stony Brook University, Stony Brook, New York 11794, USA \\ ${ }_{2}^{2}$ Perimeter Institute for Theoretical Physics, Waterloo, Ontario N2L 2Y5, Canada
}

(Received 18 December 2020; revised 12 November 2021; accepted 15 November 2021; published 13 January 2022)

\begin{abstract}
We discuss several low-energy backgrounds to sub-GeV dark-matter searches, which arise from highenergy particles of cosmic or radioactive origin that interact with detector materials. We focus, in particular, on Cherenkov radiation, transition radiation, and luminescence or phonons from electron-hole pair recombination and show that these processes are an important source of backgrounds at both current and planned detectors. We perform detailed analyses of these backgrounds at several existing and proposed experiments based on a wide variety of detection strategies and levels of shielding. We find that a large fraction of the observed single-electron events in the SENSEI 2020 run originate from Cherenkov photons generated by high-energy events in the Skipper charge coupled device and from recombination photons generated in a phosphorus-doped layer of the same instrument. In a SuperCDMS HVeV 2020 run, Cherenkov photons produced in printed-circuit boards located near the sensor likely explain the origin of most of the events containing 2-6 electrons. At SuperCDMS SNOLAB, radioactive contaminants inside the Cirlex located inside or on the copper side walls of their detectors produce many Cherenkov photons, which could dominate the low-energy backgrounds. For the EDELWEISS experiment, Cherenkov or luminescence backgrounds are subdominant to their observed event rate but could still limit the sensitivity of their future searches. We also point out that Cherenkov radiation, transition radiation, and recombination could be a significant source of backgrounds at future experiments aiming to detect dark matter via scintillation or phonon signals. We also discuss the implications of our results for the development of superconducting qubits and low-threshold searches for coherent neutrino scattering. Fortunately, several design strategies to mitigate these backgrounds can be implemented, such as minimizing nonconductive materials near the target, implementing active and passive shielding, and using multiple nearby detectors.
\end{abstract}

DOI: 10.1103/PhysRevX.12.011009

Subject Areas: Cosmology, Particles and Fields

\section{INTRODUCTION}

The search for the particle nature of dark matter is currently among the most important topics in particle physics. Direct-detection experiments provide an essential approach in our endeavor to detect dark-matter particles in the laboratory and identify their interactions. In the past decade, searches for dark-matter particles with masses in the previously unexplored range between approximately $1 \mathrm{meV}$ to $1 \mathrm{GeV}$ have gained significant attention due to novel theoretical and experimental developments [1-76]. Early experimental successes in probing dark matter interacting with electrons or nuclei in parts of this mass range have come from XENON10 [2,3], XENON100 [20,24], SENSEI [35,46,68], DarkSide-50 [37], DAMIC [47], XENON1T [63,69], SuperCDMS [36,71,72],

Published by the American Physical Society under the terms of the Creative Commons Attribution 4.0 International license. Further distribution of this work must maintain attribution to the author(s) and the published article's title, journal citation, and DOI.
EDELWEISS [48,70], and CRESST-III [33,61,62], and rapid experimental progress is expected in this decade. However, a common feature among all these experiments is that they observe sizable rates of low-energy background events of unknown origin that affect the reach of darkmatter searches. These backgrounds need to be identified, characterized, and mitigated to ensure the success of the low-mass dark-matter search program in the coming decade as experiments improve their sensitivity.

The research toward identifying the origins of several of these backgrounds is already underway, even if a satisfactory understanding of the observed events in each case is still lacking. For example, for the single-electron events in noble liquid detectors (e.g., XENON10/100/1T and DarkSide-50), several hypotheses have been proposed, such as the delayed release of electrons from the liquidgas interface, impurities capturing and releasing electrons, the photoelectric effect from photons hitting metallic surfaces, and others [77-83]. The low-energy excess in CRESST-III is thought to arise from cracking or microfracturing of the crystal or support holders that occurs when the detectors are being tightly clamped [84], but it remains 
unclear whether this explains the excess. Some singleelectron events currently seen in Skipper charge coupled device (Skipper CCD) detectors are known to originate from spurious charge generation during readout and can be reduced by optimizing the voltages that move the charge pixel to pixel, but a large single-electron-event rate remains even after subtracting the spurious-charge contribution [68]. A leakage current likely sources the singleelectron events in the SuperCDMS HVeV detectors and EDELWEISS sub-GeV dark-matter search [36,70,71], but its microphysical origin is not satisfactorily understood, nor is the microphysical origin understood of the events containing more than one electron. Other backgrounds that have been considered to produce low-energy events are the low-energy tail of the Compton and beta-decay spectra, as well as coherent solar neutrino-nucleus scattering (see, e.g., Refs. $[10,34,85])$, but in practice these backgrounds are subdominant at many experiments, and, in fact, these tails cannot explain the aforementioned excesses of low-energy events.

In this paper, we take a significant step toward identifying several processes that produce backgrounds in lowmass dark-matter detectors. In particular, we discuss and demonstrate the importance of three largely unexplored processes in the context of low-energy-threshold detectors, which arise when high-energy particles such as radiogenic electrons or gamma rays and cosmic muons interact with detector materials: Cherenkov radiation, [86] transition radiation, and luminescence or phonons from recombination. We provide a schematic depiction on how these backgrounds are obtained in detectors in Fig. 1. The Cherenkov process is realized when charged particles go through dielectric materials at velocities exceeding the speed of light in the medium, while transition radiation is obtained if charged tracks encounter interfaces separating media with different dielectric properties. As a result of these two processes, one or more photons with energies below a few eV can be emitted by the high-energy particles. Recombination photons or phonons, on the other hand, arise since high-energy particles efficiently deposit energy in materials by exciting a large number of $e^{-} h^{+}$pairs. These pairs subsequently recombine radiatively or nonradiatively, releasing an energy of the order of approximately an $\mathrm{eV}$ per pair. The low-energy photons or phonons obtained by these processes can then be absorbed in the detector target, producing a measurable signal that depends on the target properties, but can be in the form of one or more electrons, photons, phonons, or magnons, which potentially mimics the low-energy signals produced by low-mass dark matter.

To demonstrate the importance of Cherenkov radiation, transition radiation, and recombination of $e^{-} h^{+}$pairs as sources of backgrounds for dark-matter detectors, the details of different experiments and their data analysis pipelines must be taken into account. With this objective, we perform background rate estimates at several current and proposed experiments, including SENSEI, SuperCDMS HVeV/CPD/SNOLAB, EDELWEISS, and CRESST-III. Different experiments employ different strategies to mitigate backgrounds arising from high-energy tracks. Detectors such as the Skipper CCDs used by SENSEI [22,35,46,68], DAMIC-M [88], and Oscura [89], which aim to read out electron-hole pairs created by dark-matter scattering, have very little timing information but excellent position resolution. In this case, events are vetoed if their position in the Si CCD is close to an observed high-energy track. Despite these vetoes, singleelectron-event backgrounds may still arise from trackinduced radiation (which creates $e^{-} h^{+}$pairs upon absorption in the CCD), if the photons travel far away from their originating tracks. In the SENSEI CCD, this happens when the photons have energies close or below the Si band gap.
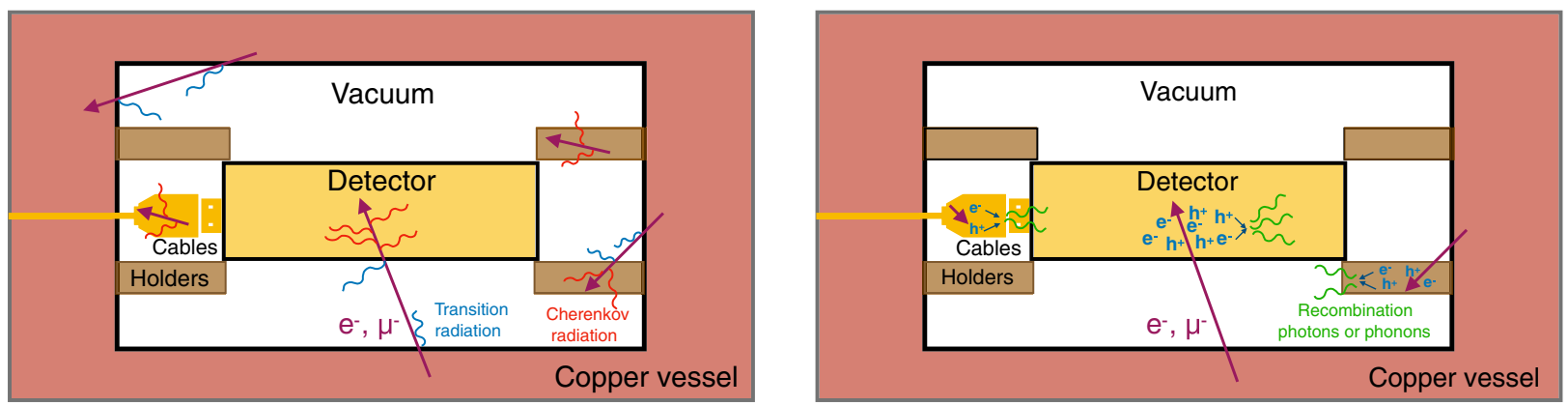

FIG. 1. Schematic for the production of radiative backgrounds: Cherenkov and transition radiation (left) and recombination photons and phonons (right). High-energy electrons (from ambient radioactivity or radioactive contaminants) and cosmic-ray muons (purple) can (i) interact in nonconductive materials, including the detector target and the surrounding materials, such as holders and cables, to produce Cherenkov radiation (red); (ii) cross surfaces to produce transition radiation (blue); and (iii) deposit energy to create luminescence or phonons via, e.g., the recombination of electron-hole pairs (green). The low-energy photons or phonons obtained by these processes can then be absorbed in the detector target, producing a measurable signal that depends on the target properties but that can take the form of, e.g., one or more electrons, photons, or phonons, which can mimic the low-energy signals produced by low-mass dark matter interacting with the detector target. 
We show that such photons can be created by the Cherenkov effect as tracks pass through the CCD or other surrounding dielectric materials and that they are also abundantly obtained from the radiative recombination of $e^{-} h^{+}$pairs created by tracks that pass through a layer on the CCD's back side with high phosphorus doping (in other CCD regions, the radiative recombination rate is comparatively smaller). By performing a quantitative analysis of the number of events obtained by these processes, we find that Cherenkov and recombination radiation are responsible for at least a large fraction of the single-electron-event rate reported by SENSEI.

Most other solid-state detectors, including SuperCDMS, EDELWEISS, and CRESST-III and proposed detectors based on scintillation [12] or single-phonon [29] signals such as SPICE [90], have excellent timing resolution. In this case, low-energy events coming from high-energy tracks that pass through the detector target are easily vetoed due to their time coincidence with the observed track. [91] However, high-energy events interacting in uninstrumented materials that surround the target usually cannot be vetoed, and, hence, low-energy photons produced in those interactions (either from Cherenkov or transition radiation or from luminescence) provide a low-energy signal. In this context, our estimates indicate that, at SuperCDMS-HVeV, Cherenkov radiation from tracks passing through printedcircuit boards (PCBs) located around the detector is very possibly the dominant source of the low-energy events containing 2-6 electrons seen in the data $[36,71]$. We also show that transition radiation provides another, smaller contribution. At EDELWEISS [70], we find that, given its well-shielded and underground location, track-induced backgrounds from any of the processes discussed here are currently expected to be subdominant but may be relevant to assess the future sensitivity of this experiment. Track-related backgrounds are also expected to be subdominant at CRESST-III $[61,62]$, since both the detector and surrounding materials are designed to scintillate to veto events associated with tracks. At SuperCDMS CPD [72], we find that Cherenkov or transition radiation, or prompt radiation from radiative recombination due to the passage of high-energy tracks, makes up less than approximately $10 \%$ of their large observed low-energy event rate. The fact that we find track-related radiative backgrounds to be subdominant to the observed background rates at EDELWEISS, CRESST-III, and SuperCDMS-CPD is consistent with the characteristics of the observed backgrounds: Most of them seem to be "low yield"; i.e., they produce little or no ionization (and, hence, cannot originate from the absorption of an above-band-gap photon) and instead produce a heat or phonon signal $[61,62,70,72,93,94]$. Nevertheless, our analysis indicates that track-related backgrounds are an important background for future versions of these detectors, at least once the low-yield backgrounds are understood. Regarding proposed detectors such as SPICE, we point out that Cherenkov or recombination radiation generated in materials surrounding the detector, or recombination phonons generated in the detector holders, could potentially be a significant source of backgrounds that had not been previously considered in the literature.

Going beyond dark-matter detectors, the above backgrounds may be relevant for other devices that have $\mathrm{eV}$ or sub-eV sensitivity. For example, neutrino detectors based on Skipper CCDs such as CONNIE [95] and $\nu$ IOLETA [96] may suffer from these backgrounds. More interestingly, these backgrounds may be important for quantum computing. A superconducting quantum bit (qubit) typically has an energy gap of $O(\mathrm{meV})$. Photons above this energy can break Cooper pairs in superconductors and create quasiparticles. This may be a source of the observed loss of coherence in superconducting qubits [97-100].

While the arguments and estimates that we perform clearly demonstrate that Cherenkov radiation, transition radiation, and recombination constitute important backgrounds, we also show that it is often surprisingly challenging to evaluate their magnitude precisely. In particular, this requires knowing or measuring the precise optical properties of all materials present in the detector and performing detailed simulations of the high-energy backgrounds, detector setup, and data analysis chain. With that being said, the required measurements and simulations are feasible, so the backgrounds discussed here can and need to be characterized precisely in the future.

Finally, and very importantly, having identified these backgrounds and their exact origin at detectors, we present specific and concrete strategies that can be implemented at future experiments in order to mitigate their impact.

The remaining paper is organized as follows. In Secs. II and III, we discuss in detail Cherenkov radiation and transition radiation produced directly by charged particles interacting in a variety of materials typically found in detectors, respectively. In Sec. IV, we discuss luminescence and phonons that are produced from the recombination of $e^{-} h^{+}$pairs after the passage of a high-energy particle in different materials. In Sec. V, we show how these radiative backgrounds contribute to the events observed in recent results from SENSEI [68], SuperCDMS HVeV [71], EDELWEISS [70], CRESST-III [61,62], SuperCDMS CPD [72], and EDELWEISS-Surf [48]. Section VI discusses these backgrounds in the upcoming SuperCDMS SNOLAB experiment [21], future Skipper CCD detectors (SENSEI at SNOLAB, DAMIC-M, and Oscura), and future detectors searching for photons or phonons (including SPICE [90]). Apart from dark-matter detections, we point out in Sec. VII that these radiative backgrounds may also be relevant for neutrino experiments and superconducting qubits. In Sec. VIII, we discuss how these radiative backgrounds can be mitigated. We summarize our findings in Sec. IX. Several Appendixes include additional details on semiconductor properties (Appendix A), as well as our background estimates for SENSEI (Appendix B), 
SuperCDMS HVeV (Appendix C), and SuperCDMS at SNOLAB (Appendix D).

\section{CHERENKOV RADIATION}

In this section, we discuss the emission of Cherenkov radiation from high-energy charged particles traversing a material. This can produce low-energy photons, which can be absorbed in the target to produce low-energy events that mimic a dark-matter signal.

\section{A. The Cherenkov effect: Theory}

The Cherenkov effect is the spontaneous emission of radiation by a charged particle passing through a dielectric material. The emission of Cherenkov radiation depends on the properties of the dielectric, which are set by its complex refraction index $\tilde{n}$ :

$$
\tilde{n} \equiv n+i \kappa, \quad n, \kappa \in \mathbb{R} .
$$

The complex refraction index determines how light propagates in the medium: $e^{i(\omega \tilde{n} x-\omega t)}=e^{-\kappa \omega x} e^{i \omega(n x-t)}$. The real part of the refraction index, $n$, sets the phase-space velocity of light in the material, while the imaginary part $\kappa$ is the extinction coefficient. Alternatively, one can describe the material with the complex dielectric function, defined as the square of the complex refraction index, $\epsilon(\omega) \equiv \tilde{n}^{2}$, or

$$
\operatorname{Re} \epsilon(\omega) \equiv n^{2}-\kappa^{2}, \quad \operatorname{Im} \epsilon(\omega) \equiv 2 n \kappa .
$$

Emission of Cherenkov photons with frequency $\omega$ happens when the product of the square of the particle velocity times the real part of the material's dielectric function is greater than one [101,102]:

$$
v^{2} \operatorname{Re} \epsilon(\omega)>1 .
$$

Given that $v \leq 1$, a necessary condition for Cherenkov radiation to occur is $\operatorname{Re} \epsilon(\omega)>1$. Note that, for nonabsorptive materials, $\kappa=0$, and the Cherenkov condition reduces to the requirement that the charged particle velocity exceeds the speed of light in the material: $v>1 / n$.

Cherenkov radiation has particular characteristics that differentiate it from other types of radiation that arise due to the passage of charged particles through matter. While these features are most accurately described quantum-mechanically, they are already apparent in classical electrodynamics. We first discuss the classical results and postpone commenting on quantum-mechanical corrections to the end of this section.

We begin by discussing the Cherenkov emission rate. The differential emission rate of Cherenkov photons by a unit-charge particle passing through a medium is given by [101] $\frac{d^{2} N_{\gamma}}{d \omega d x}=\alpha\left(1-\frac{\operatorname{Re} \epsilon(\omega)}{v^{2}|\epsilon(\omega)|^{2}}\right), \quad v^{2} \operatorname{Re} \epsilon(\omega)>1$,

where $N_{\gamma}$ is the number of emitted photons, $\omega$ is the photon frequency, and $d x$ is the charged particle's path-length differential. Given that the Cherenkov process arises at leading order in electrodynamics, the rate Eq. (2.4) is proportional to one power only of the fine-structure constant $\alpha$. [103] This must be compared, for example, to bremsstrahlung, which originates from the collisions of the charged particles with the fixed ions and, therefore, arises at $\mathcal{O}\left(\alpha^{3}\right)$ [104]. As a consequence, for photon frequencies where the condition Eq. (2.3) is fulfilled, the Cherenkov effect is expected to be one of the leading means by which charged particles radiate in the material. Note that the differential rate depends on the properties of the material through its dielectric function. However, for materials that are strongly dielectric $|\epsilon(\omega)| \gg 1$ (within some range of photon frequencies), and if the charged particle is fast enough, the condition $v^{2}|\epsilon(\omega)| \gg 1$ is satisfied. In this case, the differential rate Eq. (2.4) becomes insensitive to the details of the material and reduces to a constant $\left(d^{2} N / d \omega d x\right) \simeq \alpha$ that is independent of the photon frequency. In this limit, the total number of photons emitted within a frequency interval is simply proportional to the path length traveled by the charged particle times the frequency interval.

The angular distribution of Cherenkov photons is sharply peaked at an angle $\theta_{\mathrm{Ch}}$ with respect to the charged particle's momentum vector, which is [105]

$$
\cos \theta_{\mathrm{Ch}}=\frac{\sqrt{\operatorname{Re} \epsilon(\omega)}}{v|\epsilon(\omega)|} .
$$

Finally, Cherenkov radiation is linearly polarized, with the polarization vector lying in a direction parallel to the plane containing the charged particle and photon momentum vectors.

We conclude this section by commenting on the quantum corrections to the classical description of Cherenkov radiation discussed up to now. Quantum corrections must be considered when the time over which radiation is emitted is smaller than the inverse photon frequency: $\omega t<$ 1 [107]. In this case, quantum effects introduce a spread in the Cherenkov angle (2.5) and modifications to the rate Eq. (2.4) (the polarization remains as in the classical description). In order to estimate the size of these corrections, consider a high-energy electron track going through $100 \mu \mathrm{m}$ of $\mathrm{Si}$ at the speed of light, over a time $t \sim 3 \times 10^{-13} \mathrm{~s} \sim 500 \mathrm{eV}^{-1}$. Considering emission of Cherenkov photons in the $1-\mathrm{eV}$ range, relevant for current light-dark-matter detectors, this leads to $\omega t \geq 500$, so the classical condition $\omega t \gg 1$ is satisfied and quantum corrections are expected to be small, of the order of $1 /(\omega t) \sim \mathcal{O}(0.2 \%)$. However, note that when taking shorter 
charged tracks, or Cherenkov photons with much smaller energies, quantum corrections need to be taken into account. For instance, for photon frequencies of the order of $10 \mathrm{meV}$, which are relevant for future single-phonon detectors (see Sec. VID), quantum corrections to the classical Cherenkov formulas can be of the order of approximately $\mathcal{O}(20 \%)$.

\section{Dielectric properties of detector materials}

In order to discuss the relevance of the Cherenkov effect for dark-matter detectors in more detail, the specifics of the dielectric materials in the detectors need to be studied. While there is a variety of relevant materials, for concreteness let us now discuss the case of three semiconductors [germanium $(\mathrm{Ge})$, silicon $(\mathrm{Si})$, and gallium arsenide $(\mathrm{GaAs})$ ] and two band-gap insulators [silicon dioxide $\mathrm{SiO}_{2}\left(\alpha\right.$-quartz) and aluminum oxide $\mathrm{Al}_{2} \mathrm{O}_{3}$ (sapphire)]. Spanning more than an order of magnitude in their room-temperature band gaps, with $E_{g}^{\mathrm{Ge}}=0.66 \mathrm{eV}$, $E_{g}^{\mathrm{Si}}=1.11 \mathrm{eV}, \quad E_{g}^{\mathrm{GaAs}}=1.43 \mathrm{eV}, \quad E_{g}^{\mathrm{SiO}_{2}}=8.9 \mathrm{eV}$, and
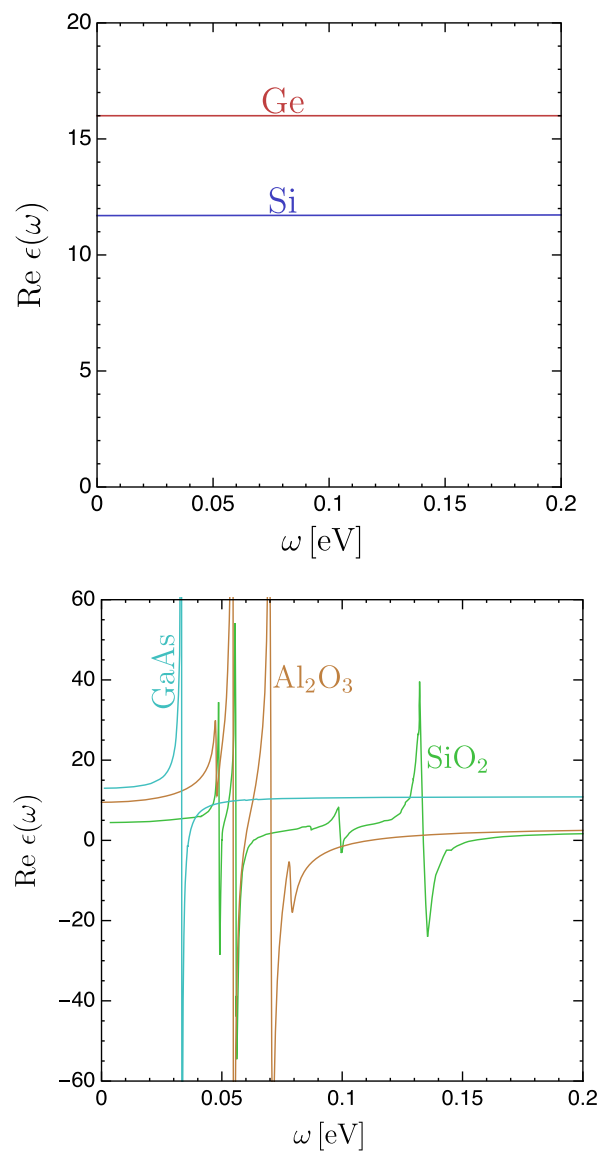

$E_{g}^{\mathrm{Al}_{2} \mathrm{O}_{3}}=8.8 \mathrm{eV}[108-110]$, these materials are representative of a wide range of nonconducting media and allow us to discuss Cherenkov radiation and photon absorption with enough generality. These materials can be found in a variety of current and planned experiments, such as SENSEI [22], SuperCDMS [111], EDELWEISS [70], DAMIC [112], and proposed athermal phonon detectors [55].

The room-temperature, experimentally measured dielectric functions for our benchmark materials are shown in Figs. 2 and 3. We separately plot the dielectric functions for energies above and below $\omega=0.2 \mathrm{eV}$ to highlight the different processes relevant at high and low frequencies: At small energies, the dielectric behavior of the materials is set by the lattice vibrations and electronic dynamics within each band, while at higher energies electronic interband transitions become relevant [113]. Our materials can be further classified in two groups: polar and nonpolar. Sapphire, $\alpha$-quartz, and GaAs are polar materials (i.e., materials that have oppositely charged ions in the primitive cells). We present their dielectric functions in the bottom panels in Figs. 2 and 3. Ge and Si are nonpolar, and their
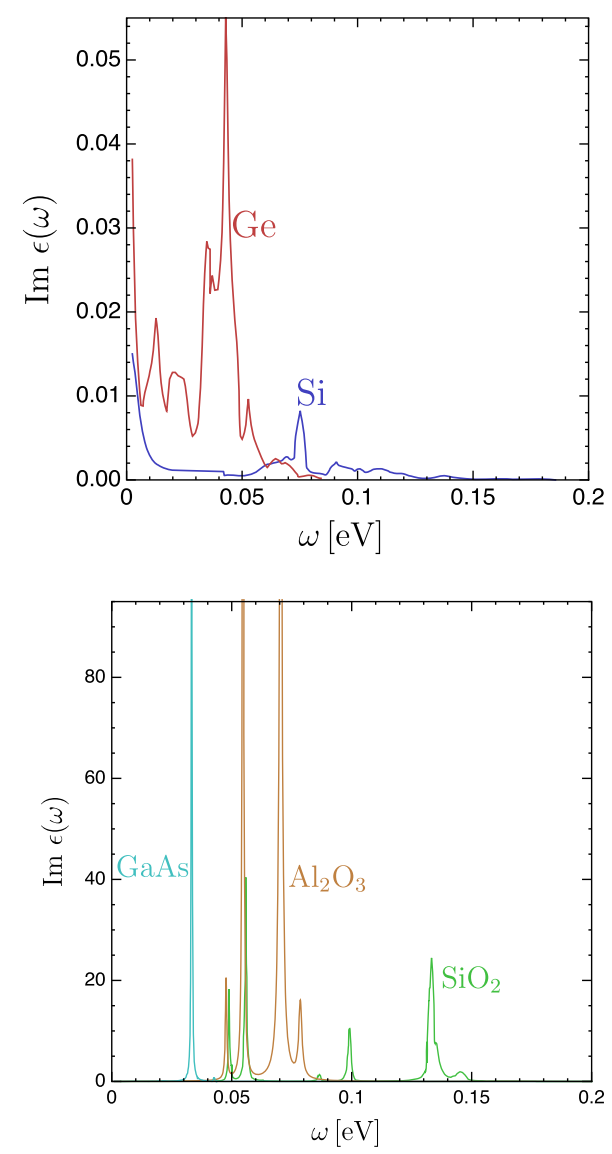

FIG. 2. Real and imaginary parts of the dielectric functions of Ge and Si (top) and of the polar materials $\alpha$-quartz, sapphire, and GaAs (bottom), in the range of photon energies $0 \leq \omega \leq 0.2 \mathrm{eV}$. For the case of sapphire and $\alpha$-quartz, which are optically anisotropic materials, we show only the dielectric function for the ordinary ray, with polarization perpendicular to the optical axis. All data are presented at room temperature. Data for Si are obtained from Refs. [114,115], for Ge from Refs. [114,116], for $\alpha$-quartz from Refs. [114,117], for sapphire from Ref. [118], and for GaAs from Ref. [114]. 

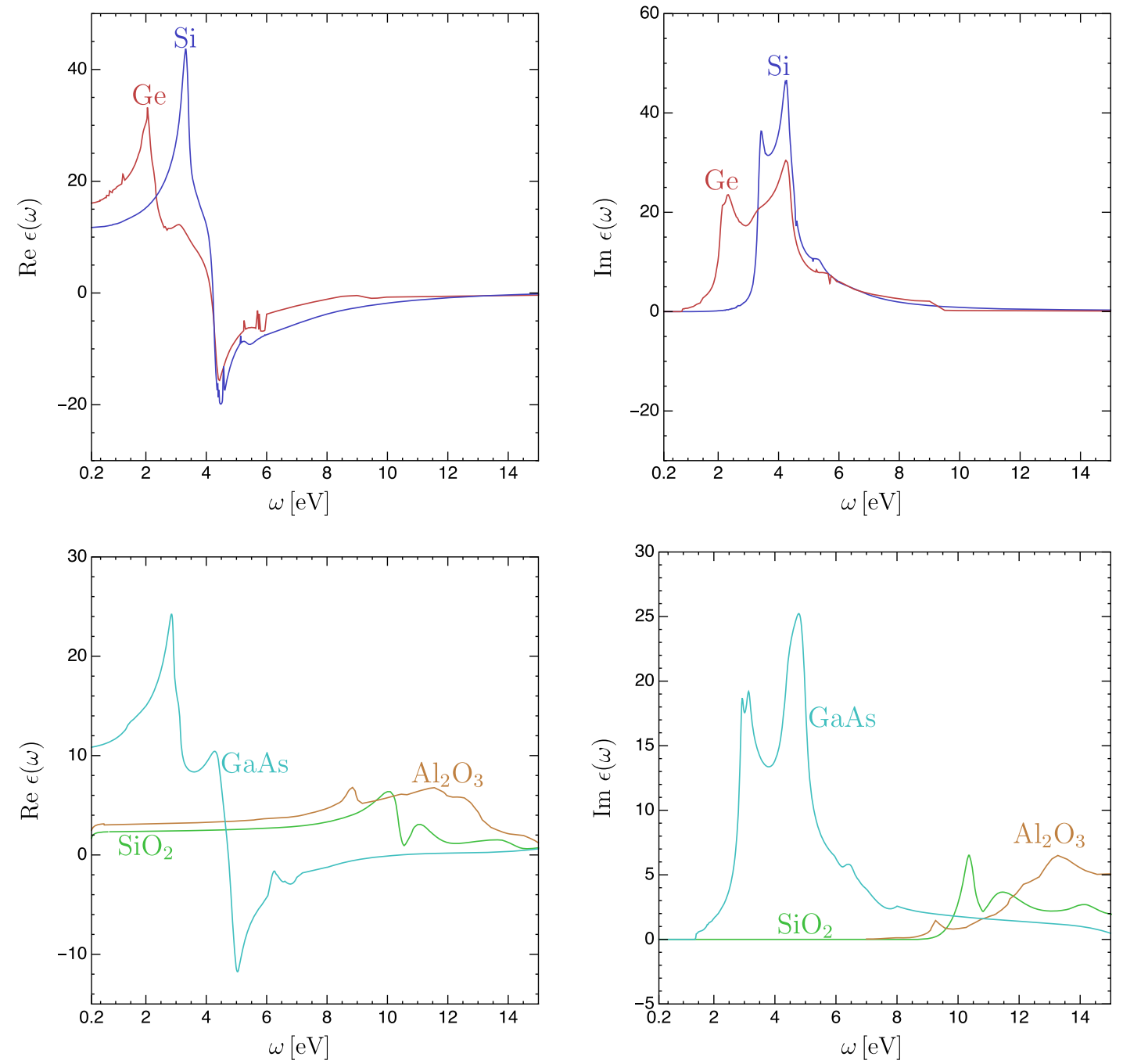

FIG. 3. Real and imaginary parts of the dielectric functions of Ge and Si (top) and of the polar materials $\alpha$-quartz, sapphire, and GaAs (bottom), in the range of photon energies $0.2 \leq \omega \leq 15 \mathrm{eV}$. For the case of sapphire and $\alpha$-quartz, we show the dielectric function for the ordinary ray. All data are at room temperature. Data for Si, Ge, $\alpha$-quartz, and GaAs are obtained from Ref. [114]. Data for sapphire are obtained from Refs. [114,119].

dielectric functions are shown in the upper panels of the same figures.

Polar materials have phonons that react strongly to light due to dipole interactions. The effect of these interactions in the dielectric function is evident at energies $\omega \leq 0.2 \mathrm{eV}$ (cf. Fig. 2, bottom left), where we see strong peaks on both the real and imaginary parts of the dielectric functions, which are due to resonant conversion of photons into single optical phonons. Nonpolar materials do not have optically active phonon modes that can be resonantly produced from photon absorption. Thus, such materials respond more weakly to light, and, correspondingly, they present only small peaks in $\operatorname{Im} \epsilon(\omega)$ (cf. Fig. 2, upper right), which are due to multiphonon processes. It is also relevant to point out that, in the infrared, multiphonon processes allow dielectrics to be absorbing up to energies that are considerably larger than single-optical phonon energies. For instance, while optical phonons in Si have energies close to $60 \mathrm{meV}$, threephonon processes involving one transverse-optical (TO) and two longitudinal-optical (LO) phonons in Si allow for photon absorption up to at least approximately $160 \mathrm{meV}$ [113].

In the range of energies above the lattice modes but much below the electronic band gaps of all our dielectric materials, $0.2 \mathrm{eV} \lesssim \omega \ll E_{g}$, their dielectric functions are approximately real and constant. This constant is called the highfrequency dielectric function $\epsilon_{\infty}$. The high-frequency dielectric function depends mostly on the electron dynamics within bands and for our different materials is $\epsilon_{\infty}^{\mathrm{Si}}=12, \epsilon_{\infty}^{\mathrm{Ge}}=16$, $\epsilon_{\infty}^{\mathrm{SiO}_{2}}=2.4, \epsilon_{\infty}^{\mathrm{Al}_{2} \mathrm{O}_{3}}=3.1$, and $\epsilon_{\infty}^{\mathrm{GaAs}}=16[114,120]$. Note that $\epsilon_{\infty}$ is larger in $\mathrm{Si}, \mathrm{Ge}$, and $\mathrm{GaAs}$ than in quartz and sapphire. The reason is that $\mathrm{Si}, \mathrm{Ge}$, and $\mathrm{GaAs}$ are covalent materials, where electrons are shared between atoms. Since these electrons are loosely bound to the lattice, the material is then highly polarizable, leading to their large $\epsilon_{\infty}$ [121]. 
Quartz and sapphire, on the other hand, have ionic bonds, in which case electrons are strongly bound to each oppositely charged ion and the material is hard to polarize, leading to small $\epsilon_{\infty}$. The larger $\epsilon_{\infty}$ in $\mathrm{Si}, \mathrm{Ge}$, and GaAs can also be explained by means of their small band gaps. In materials with small band gaps, higher-order perturbation theory effects on photon propagation in the material come with small-energy denominators, which lead to more polarizability [121]. Note also that, in the range $0.2 \mathrm{eV} \lesssim \omega \ll E_{g}$, $\operatorname{Im} \epsilon(\omega) \simeq 0$, so all our materials are transparent, as photons are too energetic to convert into either a single or multiple phonons but too soft to excite electrons to the conduction band.

For higher photon frequencies, above the band-gap energies of each material $\omega>E_{g}$, direct and indirect electronic interband transitions become energetically allowed. Correspondingly, in Fig. 3, we observe strong absorption peaks in $\operatorname{Re} \epsilon(\omega)$ and $\operatorname{Im} \epsilon(\omega)$ due to these transitions.

Throughout this section, we present optical data at room temperature, which we find to be most widely available for different materials at different photon energies. Lowering the material's temperature has two effects on the dielectric functions. First, it leads to $\mathcal{O}(1)$ changes in the values of the dielectric constant itself. For instance, in the infrared band, the dielectric function of Si decreases by a factor of approximately 2 when lowering the temperature from room temperature to $20 \mathrm{~K}$ [115]. Second, it changes other material properties that affect its optical behavior, such as the band gap. A change in the band gap leads to shifts of the dielectric function's dependence on photon frequency. A discussion on the dependence of the band-gap energy of semiconductors can be found in Ref. [108], while references to measurements of dielectric properties of materials at different temperatures can be found in Ref. [114].

\section{Cherenkov radiation in detector materials}

Inspecting Figs. 2 and 3, we see that in all our materials there are several ranges of photon energies with $\operatorname{Re} \epsilon(\omega)>1$, where Cherenkov radiation due to the passage of charged particles is possible. In $\mathrm{Si}$ and $\mathrm{Ge}$, Cherenkov photons can be emitted with energies $\omega \lesssim 4 \mathrm{eV}$. In GaAs, photons are emitted with $\omega \lesssim 5 \mathrm{eV}$, with the exception of a very narrow region of parameter space right above its optical phonon resonant mode, around $\omega \sim 0.035 \mathrm{eV}$, where the material becomes very absorptive. A material becoming absorptive results in a decrease of the real part of the dielectric function [related to the extinction coefficient $\kappa$ by $\operatorname{Re} \epsilon(\omega)=n^{2}-\kappa^{2}$; cf. Eq. (2.2)], leading to the breaking of the necessary condition for Cherenkov radiation $\operatorname{Re} \epsilon(\omega)>1$. In $\alpha$-quartz and sapphire, the allowed energy ranges are $\omega \lesssim 14 \mathrm{eV}$ and $\omega \lesssim 15 \mathrm{eV}$, respectively, excepting narrow strips of energies above optical modes. The maximal frequency at which Cherenkov radiation can be emitted in a material is strongly correlated with its band gap, since, for energies above the band gaps, materials become strongly absorptive due to interband transitions, and the Cherenkov condition is thus broken. As a consequence, insulators, which have large band gaps, generically allow for more energetic Cherenkov radiation than semiconductors.

Given $\operatorname{Re} \epsilon(\omega)>1$, a minimal kinetic energy for electrons and muons is required in order for them to emit Cherenkov photons as they pass through the dielectrics, which can be obtained from Eq. (2.3). We present the minimal energies in Fig. 4 for $\omega \leq 0.2 \mathrm{eV}$ (left) and
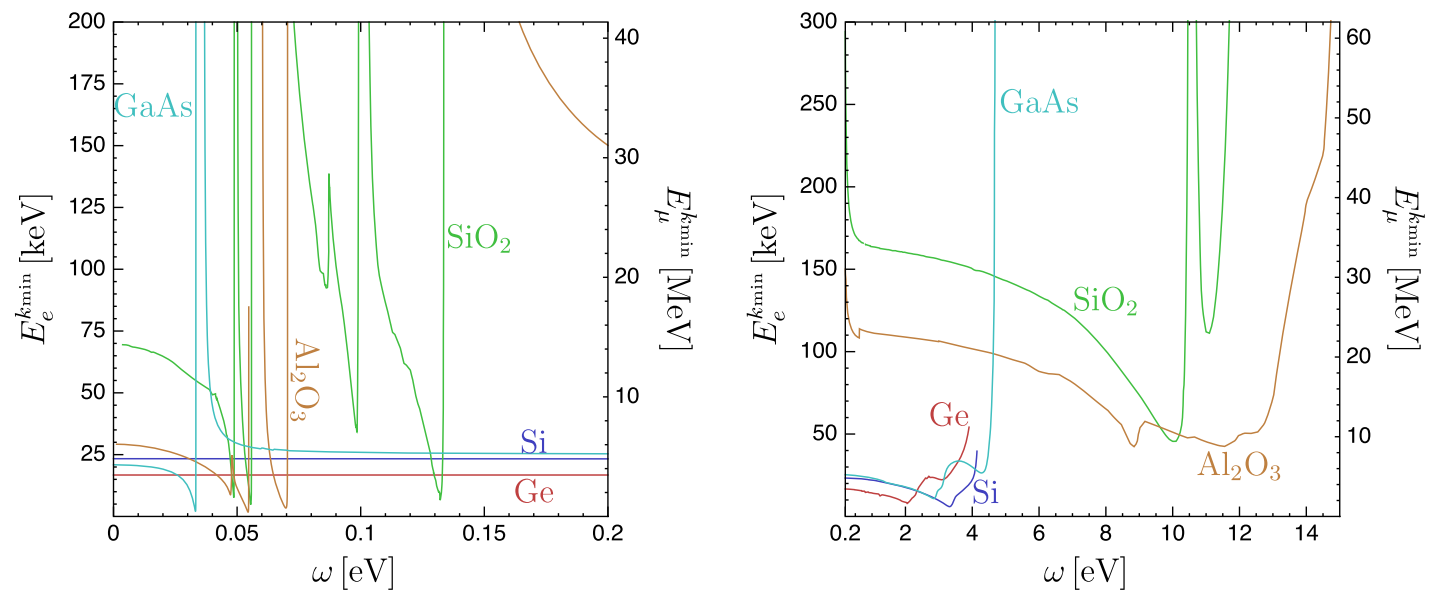

FIG. 4. Minimal electron and muon kinetic energies required to emit Cherenkov photons of frequency $\omega$ obtained from Eq. (2.3), in the frequency ranges $0 \leq \omega \leq 0.2 \mathrm{eV}$ (left) and $0.2 \leq \omega \leq 15 \mathrm{eV}$ (right), in $\mathrm{Si}, \mathrm{Ge}, \alpha$-quartz, sapphire, and GaAs. The jumps in the minimal kinetic energies in the range $\omega \leq 0.2 \mathrm{eV}$ for quartz, sapphire, and GaAs are due the strong dielectric response of polar materials for photon energies close to the lattice modes. The jump in the minimal energy for quartz at approximately $11 \mathrm{eV}$ is due to an accidental coincidence of the refraction index and the extinction coefficient at those energies, $n \sim \kappa$, that leads to a small value of Re $\epsilon(\omega)$; cf. Eq. (2.2). 
$\omega>0.2 \mathrm{eV}$ (right), for both electrons and muons. In Si and Ge, electrons (muons) with energies as low as approximately $10 \mathrm{keV}$ (approximately $2 \mathrm{MeV}$ ) emit Cherenkov radiation, while the threshold energies required in $\alpha$-quartz and sapphire are significantly larger, due the comparatively smaller dielectric function of these materials (except in narrow strips of energies close to the lattice modes).

In order to illustrate the Cherenkov radiation rate, spectrum, and the dependence on the energy of the track in concrete scenarios, let us consider the case of an electron with initial energy $E_{e}$ passing through a piece of $\mathrm{Si}$ or $\alpha$-quartz. An electron passing through a material loses energy at a rate set by the stopping power $d E / d x$, which we obtain from Ref. [122]. Thus, the spectrum of Cherenkov photons emitted by the electron before being stopped in the material is given by

$\frac{d N_{\gamma}}{d \omega}$ (per electron track) $=\int_{E_{\min }}^{E_{e}} d E \frac{d^{2} N_{\gamma}}{d \omega d x}\left[\frac{d E}{d x}\right]^{-1}$,
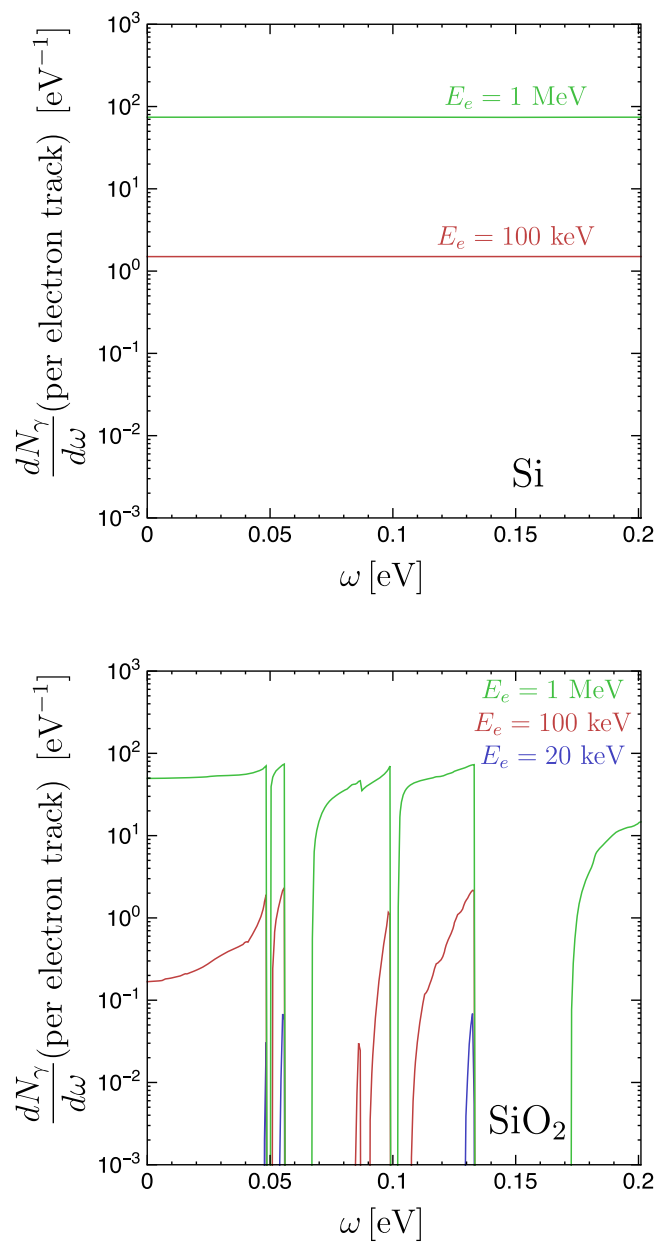

where $d^{2} N_{\gamma} / d \omega d x$ is given by Eq. (2.4). Note that, as the electron loses energy in the material, at some point its velocity falls below the threshold condition Eq. (2.3) and no more Cherenkov radiation is emitted, so the integral is cut off at the minimum energy $E_{\min }$ that satisfies the aforementioned condition.

We present the spectrum for a single-electron track for $\mathrm{Si}$ and $\alpha$-quartz in Fig. 5 for different initial energies of the electron. Consider first the case of a relativistic electron with initial energy $E_{e}=1 \mathrm{MeV}$. From the figures, we see that in this case Cherenkov radiation is emitted in a wide range of energies in both $\mathrm{Si}$ and $\alpha$-quartz. As discussed above, the main difference between the two materials is that more energetic radiation is possible in $\alpha$-quartz than $\mathrm{Si}$, given its larger band gap. In addition, at low energies $\omega \lesssim 0.2 \mathrm{eV}$, we clearly see the effect of the optically active lattice modes of $\alpha$-quartz on the radiation spectrum. In particular, right above the material's lattice modes, where the material becomes absorptive, the emission of
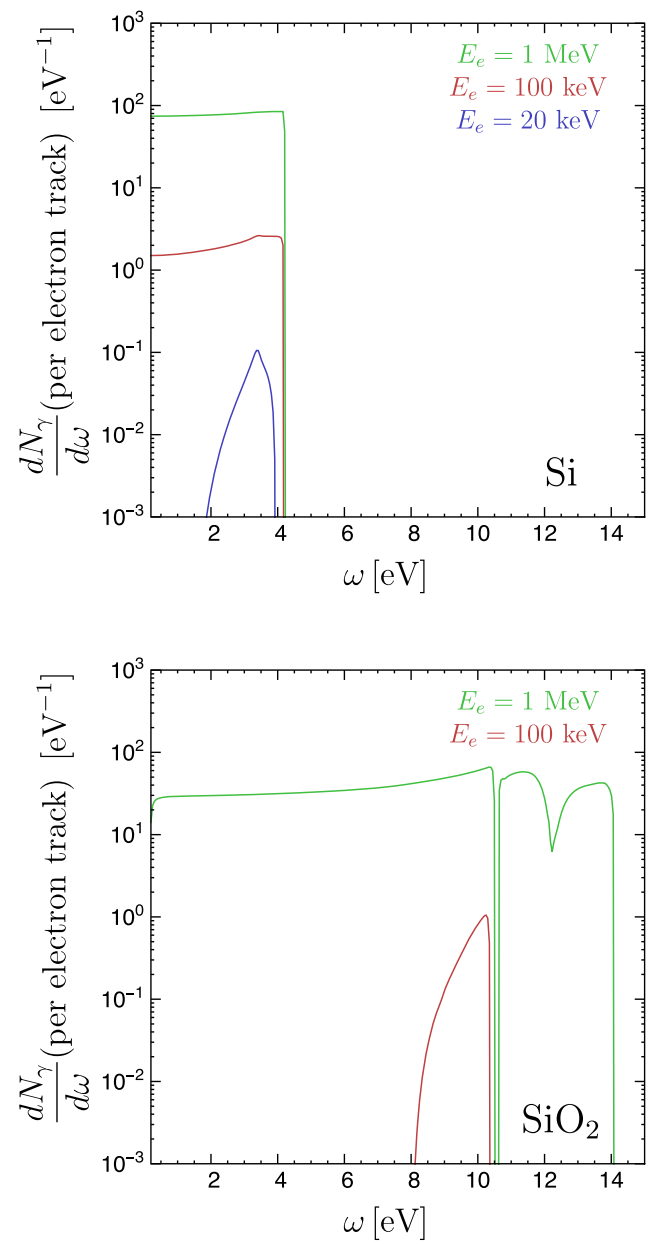

FIG. 5. Cherenkov radiation spectrum Eq. (2.6) of a single-electron track in $\mathrm{Si}$ (top) and $\mathrm{SiO}_{2}$ (bottom). We show the emission spectrum for three different electron energies: $E_{e}=20 \mathrm{keV}$ (blue line), $E_{e}=100 \mathrm{keV}$ (red line), and $E_{e}=1 \mathrm{MeV}$ (green line). For Si, there is no emission above approximately $4 \mathrm{eV}$, since $\mathrm{Si}$ becomes highly absorptive. The abrupt changes in the spectra for $\mathrm{SiO}_{2}$ at low energies correspond to peaks in its dielectric function due to a strong optical phonon response. 
Cherenkov radiation is suppressed. The spectrum in $\mathrm{Si}$, on the other hand, is rather flat for all energies below the band gap.

As we go toward lower electron energies, we observe two important effects. First and most importantly, we see a decrease of the Cherenkov radiation rate. The reason is that electrons with smaller initial energies have shorter track lengths and, thus, lead to less radiation. The length of an electron track can be characterized by its mean range, defined as the distance over which the electron travels before losing all its energy due to collisions:

$$
\ell_{e}=\int_{0}^{E} \frac{d E}{d E / d x}
$$

We show the electron mean range in Si in Fig. 6 (for other materials, the track length is simply inversely proportional to their density), where we clearly see that less-energetic electrons have shorter tracks.

The second effect of the electron track energy on the Cherenkov spectrum is that, when considering less-energetic electrons, materials either do not allow Cherenkov radiation or they do it only in specific frequency ranges where $\operatorname{Re} \epsilon(\omega)$ is large enough so that the Cherenkov condition is satisfied despite the electron's small velocity. For instance, for $E_{e}=20 \mathrm{keV}$, Cherenkov photons are emitted in $\alpha$-quartz only at energies right below its lattice modes $\omega \sim 0.05 \mathrm{eV}$ and $\omega \sim 0.13 \mathrm{eV}$, where both the dielectric function is very large due to the dynamics of its polar lattice and the material is not strongly absorptive (differently from the case of energies right above the lattice modes discussed before).

It is important to note that, even if Cherenkov photons are emitted abundantly by energetic charged particles going through dielectrics, only a very small fraction of the track's

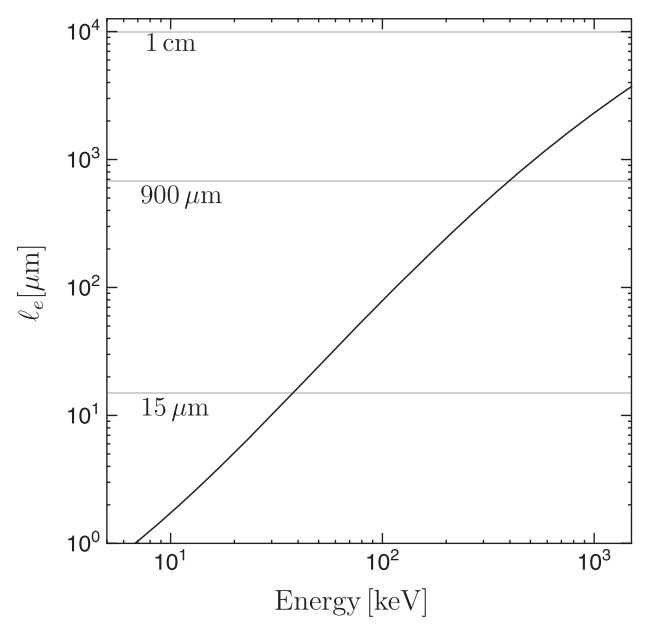

FIG. 6. Electron mean range Eq. (2.7) in Si, obtained from Ref. [122]. Horizontal lines indicate an electron mean range of 15 and $900 \mu \mathrm{m}$, which corresponds to the size of 1 and 60 pixels, respectively, of the Skipper CCDs used by SENSEI (see Sec. V). energy is lost to Cherenkov radiation. To illustrate this point, in Fig. 7 we show the percentage of the energy lost to Cherenkov radiation in different materials, for an electron with initial kinetic energy $E_{e}$ that travels in the material until losing all its energy (the situation for muons is qualitatively similar). We observe that, in all materials, less than a percent of the electron's energy is lost to Cherenkov radiation. As we discuss in Sec. IV, for the plotted range of energies, most of the electron's energy is instead lost to ionization, i.e., in the creation of electronic excitations in the material. Energy lost to radiation via other processes, such as bremsstrahlung, is also subleading for the plotted range of energies [123].

After the Cherenkov photons are emitted, the distance that they travel from the charged track before being absorbed is set by the photon attenuation length $\ell_{\gamma}$ :

$$
\ell_{\gamma} \equiv \frac{1}{2 \kappa \omega}=\frac{1}{\omega \sqrt{2|\epsilon(\omega)|-2 \operatorname{Re} \epsilon(\omega)}}
$$

where $\kappa$ is defined in Eq. (2.1) and in the last equality we make use of Eq. (2.2). When $\operatorname{Im} \epsilon(\omega)=0$, the attenuation length is infinite, and the material is nonabsorptive. We present the attenuation lengths for the different materials in Fig. 8 for $\omega \leq 0.2 \mathrm{eV}$ (left) and $\omega \geq 0.2 \mathrm{eV}$ (right). For energies $\lesssim 0.2 \mathrm{eV}$, in the range of the lattice modes, photons may convert into phonons in distances as short as fractions of a micron in $\alpha$-quartz and sapphire. By contrast, in $\mathrm{Ge}$ and $\mathrm{Si}$, photons generically travel much further, of the order of fractions of centimeters, before depositing their energy into phonons, since these materials are nonpolar and their lattice reacts more weakly to light. For higher energies, the right panel in Fig. 8 shows that photons with energy well above the band gaps have short

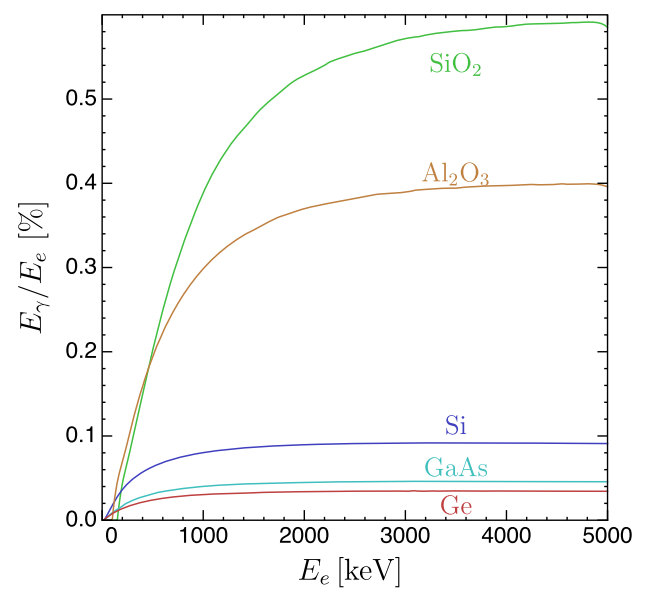

FIG. 7. Percentage of energy lost as Cherenkov radiation by an electronic track with energy $E_{e}$ in different materials. The energy lost as Cherenkov radiation is obtained by calculating the total energy radiated into Cherenkov photons up to the point where the track is stopped due to interactions with the material. 

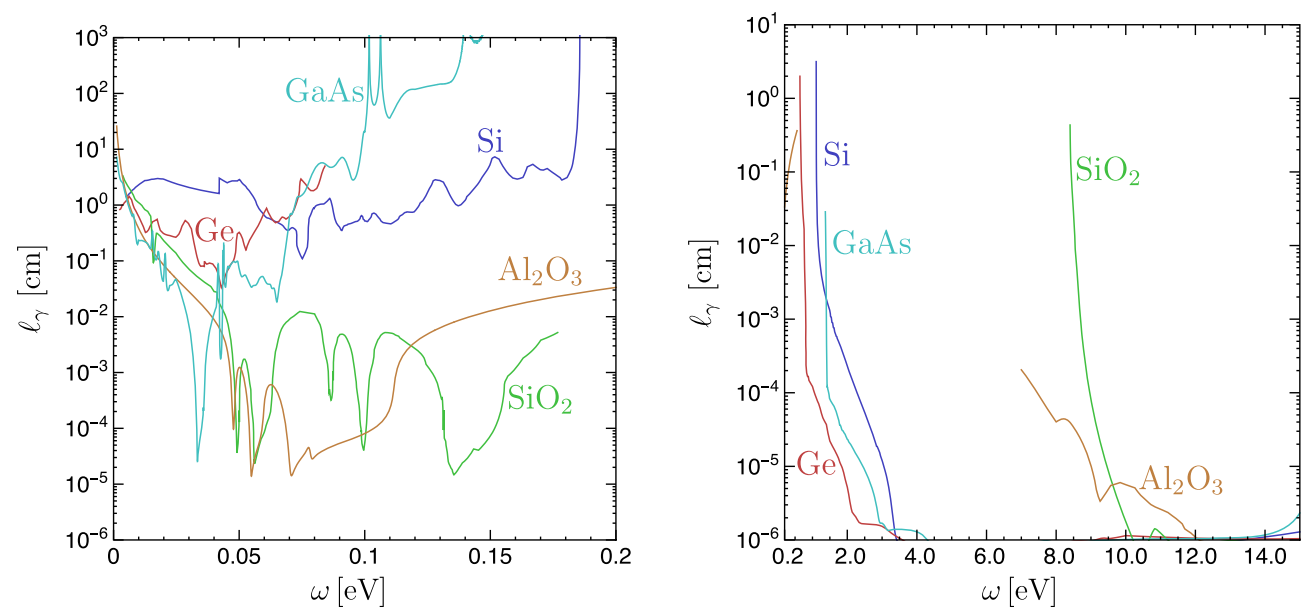

FIG. 8. Photon attenuation length [Eq. (2.8)] for photon energies $0 \leq \omega \leq 0.2 \mathrm{eV}$ (left) and $0.2 \leq \omega \leq 15 \mathrm{eV}$ (right), in different materials at room temperature. Data are taken from the same references given in Figs. 2 and 3. At energies very close to each material's band gap, the attenuation lengths presented in this figure must be taken as only approximate. Precise band-edge absorption coefficients can be found in Refs. [125-129]. For some materials, a lack of data does not allow us to indicate precise photon attenuation lengths at some energies, so gaps in the figures are noticeable. For instance, for Ge a gap in coverage in the range $0.2 \mathrm{eV} \lesssim \omega \lesssim 0.6 \mathrm{eV}$ is left between the data measured in Refs. [116,130].

absorption lengths, as they can excite electrons into the conduction band. In contrast, photons with energies very close to each material's band gap can travel over macroscopic distances before being absorbed, as interband transitions are then phase-space suppressed. Photons with energies much below the band gaps but above lattice modes are not absorbed. A photon that is not converted into an electron-hole pair or a phonon, or is not absorbed via other mechanisms such as the breaking of excitons, reaches the material surfaces and either escapes or bounces on the surfaces back into the material. Finally, we note that, in semiconductors, these properties can be significantly affected by doping; see, e.g., Ref. [124].

\section{B. Cherenkov photons as a background to dark-matter searches}

Equipped with our understanding of Cherenkov radiation in nonconducting materials, it is now easy to understand the relevance of this effect in typical low-energy-threshold darkmatter experiments. A typical detector setup has a semiconducting or insulating target that reads energy depositions into $e^{-} h^{+}$pairs or phonons. In addition to the dielectric target, other dielectric and noninstrumented materials are usually found near the detector, such as holders for the target, electronic connectors, epoxy glue, and insulating materials to cover cables. Surrounding all these elements, a metallic shielding (usually copper) is in place. Some highenergy charged tracks are expected to pass through the dielectrics of the detector, either due to particles (usually gammas, betas, or muons) that penetrate the shielding or due to radioactivity from impurities in the detector component material. These tracks lead to Cherenkov photons, which may then convert their energy into phonons or into $e^{-} h^{+}$ pairs in the detector target, mimicking the dark-matter signal.

It is likely that many $e^{-} h^{+}$pairs or phonons created by Cherenkov photons are removed by vetoing the charged track responsible for the photons. However, and as discussed above, photons may travel macroscopic distances away from the originating charged tracks, before depositing their energy into an electron-hole pair or phonons. Such long-lived photons can lead to events avoiding vetoes in two ways. First, in experiments such as SENSEI, vetoes are applied based on the distance at which the $e^{-} h^{+}$pair is created away from tracks. If this distance is large, the $e^{-} h^{+}$pair event is not vetoed, so long-lived Cherenkov photons can constitute a background. Second, at most other experiments, long-lived Cherenkov photons may originate from auxiliary noninstrumented material, escape such materials, and make it into the detector. In this case, the charged track and the corresponding Cherenkov-induced $e^{-} h^{+}$or phonon event cannot be easily vetoed. We see concrete examples of these possibilities in Sec. V.

It is also straightforward to check that Cherenkov photons are expected to be abundant within typical detector setups. For example, consider the Cherenkov radiation from a single $200 \mathrm{keV}$ electron (corresponding to a velocity $v \simeq 0.7$ ). For concreteness, we take $\mathrm{Si}$ as the medium, but the exercise can be easily repeated for other dielectric materials, and we see further examples in later sections. Since current low-energy-threshold experiments are sensitive to energy depositions down to approximately $1 \mathrm{eV}$, we consider the emission of Cherenkov photons in the energy range $1 \mathrm{eV} \leq \omega \leq 2 \mathrm{eV}$. At such frequencies, the dielectric function of $\mathrm{Si}$ is approximately real and given by $\epsilon \sim 14$, so $v \epsilon^{2} \sim 7$. To estimate the Cherenkov rate, we may then 
neglect the second term in Eq. (2.4), so the number of emitted photons is simply

$$
N_{\gamma} \sim \alpha \times \Delta \omega \times \Delta x
$$

where $\Delta \omega=1 \mathrm{eV}$ is the photon energy interval and $\Delta x$ is the electron track length. To estimate the typical charged track length, we use the electron mean range $\ell_{e}$, presented in Fig. 6. From the figure, we see that the mean range of a $200 \mathrm{keV}$ electron in $\mathrm{Si}$ is approximately $200 \mu \mathrm{m}$. Thus, the number of Cherenkov photons emitted by a single charged electron track is

$$
N_{\gamma} \sim 8\left[\frac{\Delta \omega}{1 \mathrm{eV}}\right]\left[\frac{\Delta x}{200 \mu \mathrm{m}}\right]
$$

Multiple charged tracks are expected in typical detectors due to imperfect shielding and radioactivity. For example, in the SENSEI run in the MINOS cavern [68], hundreds of charged tracks go through the CCD per g-day of exposure. Multiplying the result in Eq. (2.10) with the number of tracks, we see that thousands of $\mathcal{O}(\mathrm{eV})$ Cherenkov photons are expected per g-day.

\section{TRANSITION RADIATION}

In the previous section, we discuss the spontaneous emission of photons by charged particles going through homogeneous, nonconducting material. In the presence of inhomogeneities, additional radiation arises as tracks encounter interfaces via the Cherenkov-transition effect, also referred to as transition radiation.

\section{A. Transition radiation: Theory}

The main features of transition radiation can be discussed within its simplest realization, which occurs when a charged particle transitions between two different, semiinfinite, and homogeneous media, which we refer to as medium 1 and 2 . These media can be conductors or insulators. Assuming that the particle goes from medium 1 to 2 with a constant velocity $v$ that is normal to the surface separating the media, the forward radiation spectrum, as observed from medium 2, is [131,132]

$$
\frac{d^{2} N_{\gamma}}{d \omega d \Omega}=\frac{\alpha v^{2}}{\pi^{2} \omega} \sqrt{\left|\epsilon_{2}\right|} \sin ^{2} \theta \cos ^{2} \theta\left|\frac{\left(\epsilon_{2}-\epsilon_{1}\right)\left(1-v^{2} \epsilon_{2}-v \sqrt{\epsilon_{1}-\epsilon_{2} \sin ^{2} \theta}\right)}{\left(1-v^{2} \epsilon_{2} \cos ^{2} \theta\right)\left(1-v \sqrt{\epsilon_{1}-\epsilon_{2} \sin ^{2} \theta}\right)\left(\epsilon_{1} \cos \theta+\sqrt{\epsilon_{1} \epsilon_{2}-\epsilon_{2}^{2} \sin ^{2} \theta}\right)}\right|^{2}
$$

where $d \Omega=2 \pi \sin \theta d \theta$ is the solid-angle differential, $\theta$ is the polar angle measured relative to the normal to the surface (see Fig. 9), and $\epsilon_{1,2}$ are the complex and frequency-dependent dielectric functions of the two semiinfinite media. Transition radiation is also emitted in the backward direction. The corresponding spectrum, as seen from medium 1 and with $\theta$ being the polar angle measured from the normal pointing toward medium 1 (see Fig. 9), is

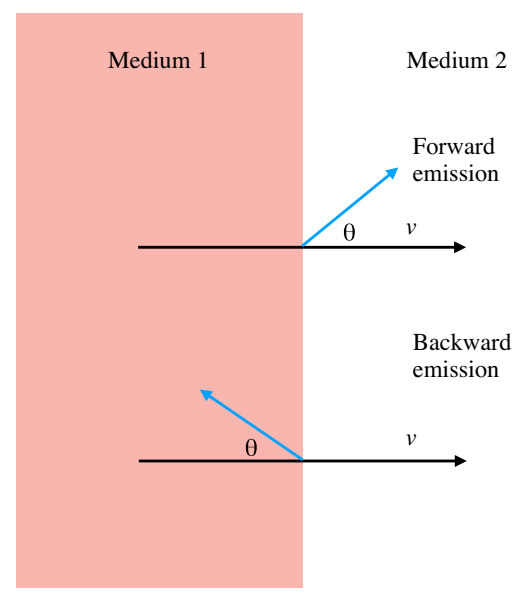

FIG. 9. Polar angle conventions for forward and backward transition radiation emission. By definition, $\theta \in[0, \pi / 2]$ in both cases. obtained by exchanging $\epsilon_{1}$ and $\epsilon_{2}$ and setting $v$ to $-v$ in Eq. (3.1).

For normal incidence on the interface, transition radiation is polarized in the plane specified by the velocity of the charged particle and the direction of the radiation. While transition radiation is an interface effect, the emission is not localized and happens instead over a formation length from the surface. This formation length is of the order of the photon wavelength for nonrelativistic particles but is significantly enhanced in the relativistic case; see Ref. [133] for discussions on the formation zone. Finally, we point out that a generalized expression for the radiation spectrum for particles going through interfaces at arbitrary incidence angles can be found in Ref. [134].

From Eq. (3.1), we see that Cherenkov-transition radiation arises at leading order in $\alpha$, similarly to the conventional Cherenkov effect seen in homogeneous media. In fact, the Cherenkov-transition effect can be regarded as a generalization of the conventional Cherenkov effect that accounts for the inhomogeneities in the media. The relation between the two processes is manifested in the poles of the emission spectrum Eq. (3.1). For nonabsorptive media with real dielectric functions, the singularities happen due to Cherenkov emission in medium 2 at the Cherenkov angle $\cos ^{2} \theta_{\mathrm{Ch}}=1 / v^{2} \epsilon_{2}$ and when the factor $1-v \sqrt{\epsilon_{1}-\epsilon_{2} \sin ^{2} \theta}$ vanishes, which corresponds to the Cherenkov radiation emitted in medium 1 and refracted 

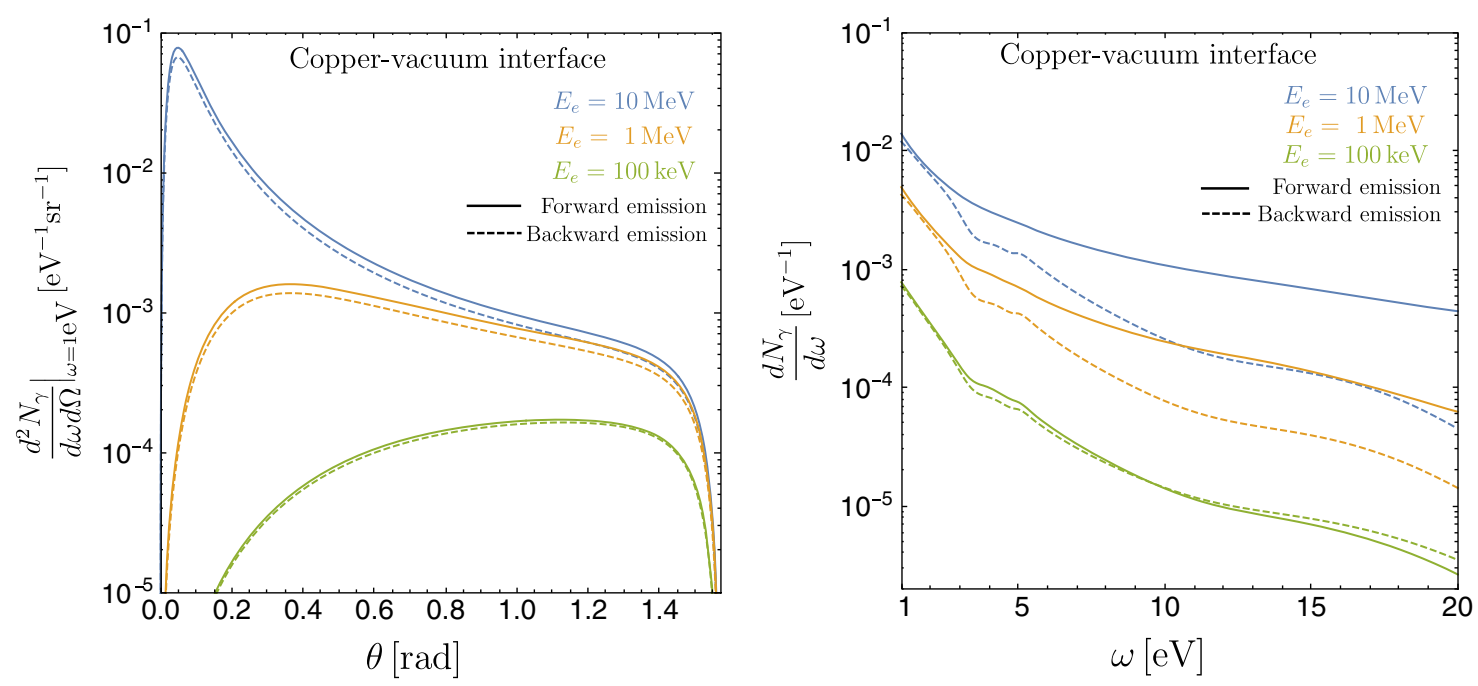

FIG. 10. The angular spectrum $d^{2} N_{\gamma} / d \omega d \Omega$ at $\omega=1 \mathrm{eV}$ (left) and the energy spectrum $d N_{\gamma} / d \omega$ (right) of transition radiation, for an electron passing a copper-vacuum interface. We present the spectra for three choices of electron energies: $E_{e}=10 \mathrm{MeV}$ (blue lines), $E_{e}=1 \mathrm{MeV}$ (orange lines), and $E_{e}=100 \mathrm{keV}$ (green lines). Solid lines show the rate for forward emission (electron moving from copper to vacuum), while dashed lines show the rate for backward emission (electron moving from vacuum to copper).

into medium 2 [135]. [136] These poles are kinematically accessible only when the Cherenkov condition Eq. (2.3) is fulfilled. When Cherenkov radiation is possible, integrating the spectrum Eq. (3.1) over angles gives a divergent result, as it includes the infinite amount of radiation emitted by the tracks as it goes through each one of the two semi-infinite homogeneous media $[133,135,137]$. In order to extract the radiative effect due to the interfaces alone, one may define a finite "pure" transition radiation contribution by subtracting the divergent pieces of the radiation along the Cherenkov angles. We refer the reader to Ref. [137] for a detailed discussion on how to extract systematically the pure transition contributions and also regulate the infinite conventional Cherenkov pieces, so that a physical spectrum is obtained. For tracks with velocities falling below the Cherenkov emission threshold, expression Eq. (3.1) is finite and uniquely due to pure transition radiation. Note that pure transition radiation can be emitted for any track velocity and in both conducting and nonconducting materials. [138] Finally, for interfaces separating only absorptive media, where the dielectric functions are complex, or separating absorptive media and vacuum (such as a metal-vacuum interface), expression (3.1) leads to a finite spectrum for all track velocities, as the poles are regulated by the absorption of the Cherenkov radiation in the material and since Cherenkov radiation is not supported in vacuum.

\section{B. Transition radiation as a background to dark-matter searches}

In order to illustrate the relevance of transition radiation for dark-matter detectors, let us now consider the simple case of a single relativistic electron track going through a copper-vacuum interface. This situation is typically encountered in experiments, since the detector target is usually enclosed in a copper vessel, with a vacuum gap left in between the target and vessel (for concrete examples, see Sec. V). We consider two cases: first, the forward emission spectrum for a track going from copper (medium 1) to vacuum (medium 2) and, second, the backward emission spectrum for a track going from vacuum (medium 1) to copper (medium 2). Using Eq. (3.1) and the dielectric function of copper from Ref. [140], we obtain the transition radiation spectrum, and we present its angular and frequency dependence in Fig. 10. From Fig. 10 (left), we see that the angular configuration of the spectrum at $\omega=1 \mathrm{eV}$ depends strongly on the track velocity, with a more forward (backward) spectrum observed at higher boost for tracks going from copper to vacuum (vacuum to copper). Such behavior can be understood by noting that, at such frequencies, the dielectric function of copper is large- $\left|\epsilon_{\mathrm{Cu}}(\omega=1 \mathrm{eV})\right| \sim 10^{2} \gg 1$-so Eq. (3.1) can be approximated to

$$
\frac{d^{2} N_{\gamma}}{d \omega d \Omega} \sim \frac{\alpha v^{2}}{\pi^{2} \omega} \frac{\sin ^{2} \theta}{\left(1-v^{2} \cos ^{2} \theta\right)^{2}} .
$$

From Eq. (3.2), we see that, in this limit, the transition radiation rate is independent of the precise value of the copper dielectric function [as long as the condition $\left|\epsilon_{\mathrm{Cu}}(\omega=1 \mathrm{eV})\right| \sim 10^{2} \gg 1$ is satisfied], and the radiation in vacuum is peaked at a forward or backward angle, as measured from the track, that is inversely proportional to the tracks' boost: $1 / \gamma\left(\gamma=1 / \sqrt{1-v^{2}}\right)$. The expression Eq. (3.2) also indicates that transition radiation from a vacuum-copper interface shows an infrared-dominated spectrum, which can also be seen in Fig. 10 (right). 


\section{LUMINESCENCE AND PHONONS FROM RECOMBINATION}

Cherenkov and transition radiation discussed in the two previous sections correspond to the direct emission of photons by charged particles passing through a material. Tracks can also excite electronic transitions in the material, which can produce low-energy quanta (photons and phonons) when electrons return to the ground state. Photons emitted by the excited material, instead of directly by the charged track, are called luminescence.

In this section, we focus on a special class of electronic excitations, which is the creation of $e^{-} h^{+}$pairs in nonconducting media, a process which in this context is also referred as ionization. This process is the main mechanism by which electrons from radioactivity and cosmic-ray muons deposit energy in solid-state detector materials [123]. In this case, phonons or photons are obtained by the relaxation and recombination of the excited pairs via a variety of mechanisms that we now explore.

Radiative and nonradiative recombination are materialdependent processes, but some of the most important dynamics relevant for a wide variety of media can be illustrated by focusing on the case of recombination in semiconductors. In what follows, we concentrate on three common semiconductors found in dark-matter detectors: $\mathrm{Si}, \mathrm{Ge}$, and GaAs.

\section{A. Recombination of $e^{-} h^{+}$pairs: Theory}

\section{Electron-hole dynamics}

A charged track passing through a material loses energy both by direct radiative processes and by the creation of excited $e^{-} h^{+}$pairs. The energy loss to $e^{-} h^{+}$pairs happens due to hard scatters that lead to energetic secondary electrons in the material, which subsequently create multiple $e^{-} h^{+}$pairs via phonon-mediated scattering [141]. The multiplication of the secondary electrons happens over their mean-free path, determined by the aforementioned phononmediated scattering in the material (which, in $\mathrm{Si}$, is of the order of $10^{-2} \mu \mathrm{m}$ [141]) and stops when the energy of the hardest electron participating in the scatterings falls below the energy required to create a new pair. As a result of this process, the track leaves behind a dense cloud of several $e^{-} h^{+}$pairs, shown schematically in Fig. 11.

The total amount of $e^{-} h^{+}$pairs created by the passage of the track is characterized by the mean ionization energy $\varepsilon$, which is defined as the average amount of energy that the hard track needs to leave in the material to create each pair. We present values of the mean ionization energy $\varepsilon$ for our benchmark semiconductors in Table I. The mean ionization energy is larger than the material's band gap, since part of the deposited energy goes into excess residual energy of the generated pairs. The excess energy can be released as the $e^{-} h^{+}$pairs go to the band edges by emitting phonons [142]. The $e^{-} h^{+}$excitation process and the relaxation of an

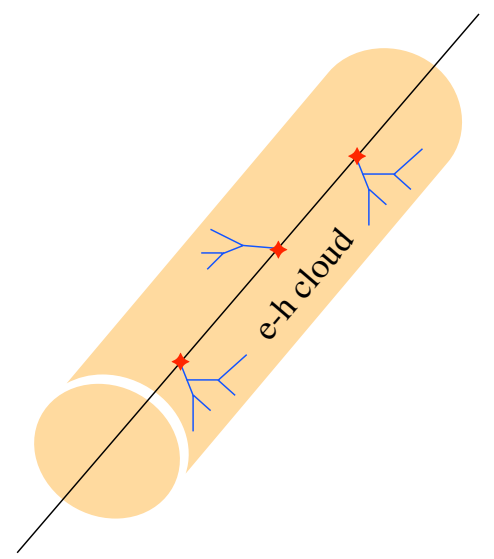

FIG. 11. Schematic depiction of the creation of $e^{-} h^{+}$pairs by a track, shown as a black line, as it passes through a material. Hard scatters of the track, shown by the red stars, create secondary energetic electrons that subsequently create more $e^{-} h^{+}$pairs. The secondary electrons and $e^{-} h^{+}$pairs are represented by the blue lines, and the cloud that they form is represented in yellow.

electron to the conduction band edge are schematically shown in Fig. 12.

In terms of the mean ionization energy, the total number of pairs created by a track traveling a distance $L$ in the material is given by

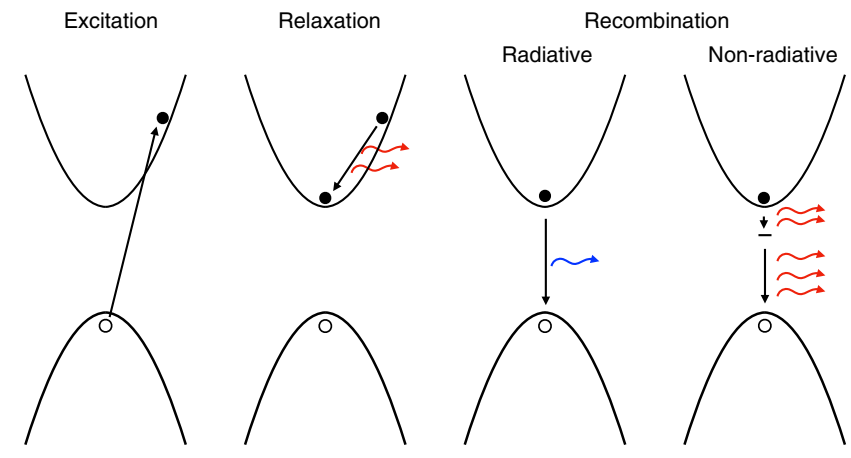

FIG. 12. Simplified band-structure representation of typical $e^{-} h^{+}$excitation, relaxation, and recombination processes. From left to right, in the first step, a scattering event excites an electron (black circle) from the valence band into the conduction band, leaving a hole (empty circle) behind. In the second step, the electron releases its excess energy in the form of phonons (red arrows) and moves to the bottom of the conduction band. Recombination of the electron and hole may then happen directly across the band gap via the emission of a near-band-gap photon (blue arrow). Another possibility, shown in the rightmost panel of the figure, is that there is an available energy level between the valence and conduction bands, referred to as a "trap," due to impurities in the material. In this case, the electron is first captured by the trap and may subsequently recombine with the hole, with the released energy usually going into multiple phonons. While we show a direct band gap, our schematic description is valid for both direct and indirect gap materials. 


$$
N_{e}=N_{h}=\left.\int_{0}^{L} d x \varepsilon^{-1} \frac{d E}{d x}\right|^{\text {ionization }}
$$

where $\left.(d E / d x)\right|^{\text {ionization }}$ is the stopping power due to ionization.

The dominant process setting the stopping power in a material depends on the track energy. In typical dark-matter experiments, especially those well shielded and underground, the majority of the tracks correspond to electrons arising from radioactivity, which have energies below a few $\mathrm{MeV}$. For such range of energies, tracks deposit their energy in nonconducting media overwhelmingly by creat-

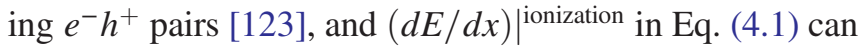
be replaced by the full stopping power $d E / d x$, first introduced in Sec. II A. When considering highly energetic tracks, such as cosmic muons with very large boosts, most of the energy loss is due to radiation instead, in which case the stopping power only due to ionization must be used in Eq. (4.1).

After the track passes by and the creation of pairs stops, the subsequent dynamics of the $e^{-} h^{+}$cloud left behind can be captured by the continuity equation

$$
\frac{\partial n_{e, h}}{\partial t}=-\nabla \cdot \vec{j}_{e, h}-\Gamma_{e, h},
$$

where $n_{e, h}$ is the electron or hole number density. The first term on the right-hand side is the electron and hole current $\vec{j}_{e, h}$, which accounts for their motion. The second term $\Gamma_{e, h}$ is the electron and hole disappearance rate density, which in the bulk material is due primarily to recombination due to defects (traps), direct recombination across the band gap, and Auger recombination. We show below that the rates for these three processes are proportional to one, two, and three powers of the $e^{-} h^{+}$densities, so they are dominant at low, intermediate, and high $e^{-} h^{+}$concentrations, respectively (see, e.g., Ref. [154] for a discussion of the dominant modes in $\mathrm{Si}$ ). We show a schematic depiction of some of the possible recombination processes in Fig. 12. In the following paragraphs, we discuss each one of the processes involved in the dynamics of the $e^{-} h^{+}$pairs in detail. Note that for brevity we do not take into account the formation and diffusion of excitons (bound states of $e^{-} h^{+}$pairs), which needs to be considered in a careful treatment. This is especially important below room temperature $[155,156]$.

\section{Diffusion and drift currents}

The electron and hole currents due to electricfield-induced drifts and diffusion are given, respectively, by [147]

$$
\begin{gathered}
\vec{j}_{e}=-n_{e} \mu_{e} \vec{E}-D_{e} \nabla n_{e}, \\
\vec{j}_{h}=n_{h} \mu_{h} \vec{E}-D_{h} \nabla n_{h},
\end{gathered}
$$

where $\vec{E}$ is the electric field and $\mu_{e, h}$ and $D_{e, h}$ are the mobility and diffusion constants, respectively, for the electron or hole. The mobility and diffusion constants are related to each other via the Einstein equation [147]

$$
D_{e, h}=\frac{\mu_{e, h} T}{e},
$$

where $e=\sqrt{4 \pi \alpha_{\mathrm{em}}} \simeq 0.3$ is the electric charge and $T$ is the material's local temperature. [157] In deriving Eq. (4.5), it is assumed that the semiconductor is nondegenerate so that Boltzmann statistics for the energy distributions of the electrons and holes can be used. A generalized version of the Einstein relation accounting for Fermi statistics can be found in Ref. [158].

The mobility and diffusion constants depend on both temperature and doping levels $[113,159]$. We summarize their room-temperature, low-doping-level values for our benchmark materials in Table I. The mobilities are also related to the material's conductivity by [147]

$$
\sigma=e\left(\bar{n}_{e} \mu_{e}+\bar{n}_{h} \mu_{h}\right)
$$

where $\bar{n}_{e, h}$ are the background electron and hole densities discussed in Appendix A.

Finally, we define the characteristic timescales for diffusion and drift processes as the periods over which the $e^{-} h^{+}$densities change by an $e$-fold due to transport, $\left|\left(\partial \log n_{e . h} / \partial t\right)^{-1}\right|$. The timescales are given by

$$
\begin{aligned}
\tau_{e, h}^{\mathrm{drift}} & \equiv \frac{1}{\mu_{e, h}} \frac{n_{e, h}}{\left|\nabla \cdot\left(n_{e, h} \vec{E}\right)\right|}, \\
\tau_{e, h}^{\mathrm{diff}} & \equiv \frac{1}{D_{e, h}} \frac{n_{e, h}}{\left|\nabla^{2} n_{e, h}\right|} .
\end{aligned}
$$

Note that the drift time can alternatively be estimated from Ohm's law: $J \equiv e n_{e, h} v_{e, h}=\sigma E$, where $v_{e, h}$ is the drift

TABLE I. Electronic properties of $\mathrm{Si}, \mathrm{Ge}$, and $\mathrm{GaAs}$ at room temperature and low doping levels. $E_{g}$ is the band gap, $\varepsilon$ the mean ionization energy, $\mu_{e, h}$ the electron and hole mobilities, $B$ the radiative recombination coefficient, and $a_{e, h}$ the electron and hole Auger coefficients. For our estimates in SENSEI's Si Skipper CCDs (see Sec. VA), which operate at $135 \mathrm{~K}$, we use $\varepsilon=$ $3.75 \mathrm{eV}$ [143].

\begin{tabular}{lccc}
\hline \hline & $\mathrm{Si}$ & $\mathrm{Ge}$ & $\mathrm{GaAs}$ \\
\hline$E_{g}(\mathrm{eV})[108]$ & 1.11 & 0.66 & 1.43 \\
$\varepsilon(\mathrm{eV})[142,144-146]$ & 3.63 & 2.8 & 4.57 \\
$\mu_{e}\left(\mathrm{~cm}^{2} / \mathrm{V} / \mathrm{s}\right)[147]$ & 1400 & 3900 & 8500 \\
$\mu_{h}\left(\mathrm{~cm}^{2} / \mathrm{V} / \mathrm{s}\right)[147]$ & 470 & 1900 & 400 \\
$B\left(\mathrm{~cm}^{3} / \mathrm{s}\right)[148-150]$ & $10^{-14}$ & $3.4 \times 10^{-14}$ & $7.2 \times 10^{-10}$ \\
$a_{e}\left(\mathrm{~cm}^{6} / \mathrm{s}\right)[151-153]$ & $2.8 \times 10^{-31}$ & $2 \times 10^{-32}$ & $1.7 \times 10^{-31}$ \\
$a_{h}\left(\mathrm{~cm}^{6} / \mathrm{s}\right)[151-153]$ & $9.9 \times 10^{-32}$ & $1.1 \times 10^{-31}$ & $2.4 \times 10^{-30}$ \\
\hline \hline
\end{tabular}


velocity of the carriers. Using the material's conductivity Eq. (4.6) in Ohm's law, the drift carrier velocity is found to be $v_{e, h}=\mu_{e, h} E$. Thus, the timescale for the carrier to transverse a distance $d$ is $\tau_{e, h}^{\mathrm{drift}} \sim d /\left(\mu_{e, h} E\right)$. This timescale coincides with the one in Eq. (4.7) upon replacement of the gradient by the inverse of the typical length scale $\nabla \cdot\left(n_{e, h} \vec{E}\right) \sim n_{e, h} E / d$.

\section{Band-to-band recombination}

The first mechanism by which electrons and holes can disappear is by band-to-band ("direct") recombination across the band gap. This process happens radiatively, via the emission of a near-band-gap photon, as shown in Fig. 12. The corresponding rate density is given by [149]

$$
\Gamma_{e}^{\text {direct }}=\Gamma_{h}^{\text {direct }}=B\left(n_{e} n_{h}-\bar{n}_{e} \bar{n}_{h}\right)
$$

where $\bar{n}_{e, h}$ are the background carrier concentrations discussed in Appendix $A$ and $B$ is the radiative recombination coefficient, discussed below. The rate in Eq. (4.8) is proportional to the product of $n_{e}$ and $n_{h}$, as both an electron and a hole are required for band-to-band recombination, and vanishes when the densities reach the background values, at which point the material stops emitting photons [160].

The radiative recombination coefficient can be related to the light absorption coefficient, defined as the inverse of the photon attenuation length $\alpha \equiv \ell_{\gamma}^{-1}$ [cf. Eq. (2.8)], using detailed balance. The result is [149]

$$
\begin{aligned}
B & \equiv \int_{0}^{\infty} d \omega \frac{d B}{d \omega} \\
& =\frac{1}{n_{i}^{2}} \int_{0}^{\infty} d \omega \frac{\alpha(\omega, T) n^{2}(\omega, T) \omega^{2}}{\pi^{2}} \frac{1}{\exp (\omega / T)-1},
\end{aligned}
$$

where $n(\omega, T)$ is the refraction index and $n_{i}$ is the intrinsic carrier concentration given in Eq. (A2) in Appendix A. In Eq. (4.9), $d B / d \omega$ is the differential recombination coefficient, which determines the recombination radiation spectrum. The expression for the recombination coefficient above must be taken only as approximate, as it neglects important corrections from the long-range Coulomb forces between electrons and holes. We refer the reader to Ref. [148] for a detailed treatment of these effects. Note that the recombination coefficient depends on temperature both explicitly and via its dependence on the dielectric properties of the material and the intrinsic carrier density. A discussion of the temperature dependence of this coefficient in Si can also be found in Refs. [148,161]. In this material, the radiative recombination coefficient increases by roughly an order of magnitude as the temperature is decreased from room temperature down to $100 \mathrm{~K}$ and by 4 orders of magnitude if the temperature is decreased to $20 \mathrm{~K}$.
This sharp increase of the radiative rate at low temperatures is due to exciton-mediated recombination [148].

We present the room-temperature radiative recombination coefficients for our benchmark semiconductors in Table I. From the table, we see that the recombination coefficient in GaAs is significantly larger than in $\mathrm{Si}$ and $\mathrm{Ge}$. This is due to the fact that GaAs is a direct-band-gap semiconductor, while $\mathrm{Si}$ and $\mathrm{Ge}$ are indirect-band-gap materials. In indirect-band-gap semiconductors, radiative recombination is suppressed, as it must be assisted by the emission of a phonon. We also show in Fig. 13 the normalized recombination spectrum $(d B / d \omega) / B$ for $\mathrm{Ge}$, $\mathrm{Si}$, and $\mathrm{GaAs}$ at zero doping and at room temperature. From the figures and as expected, we see that the radiative recombination spectrum is peaked around each material's band gap. Inspecting expression (4.9), we see that the peak arises, since the absorption coefficient is small at energies much below the band gap, while at energies much above it the recombination spectrum is exponentially suppressed. The band gap and, therefore, the position of the radiative recombination spectrum peak depend on both temperature and doping levels. The position of the peak is, thus, expected to shift toward lower frequencies when increasing doping levels and toward higher frequencies when decreasing temperatures $[108,162]$.

As for the diffusion and drift processes discussed in the previous section, we may define a timescale for the bandto-band recombination rate. The corresponding timescale is given by

$$
\tau_{e, h}^{\text {direct }} \equiv \frac{n_{e, h}}{\Gamma^{\text {direct }}}
$$

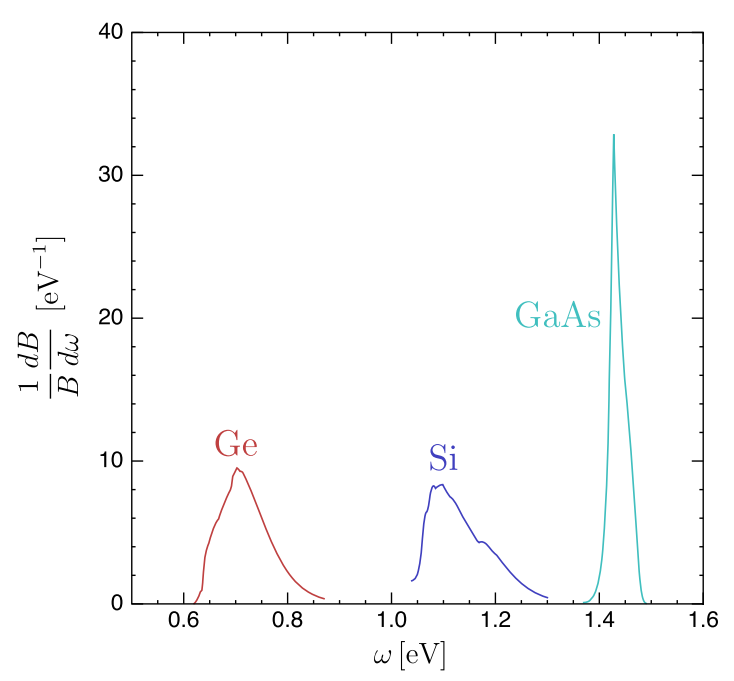

FIG. 13. Normalized spectrum of photons $(d B / d \omega) / B$ emitted by the recombination of $e^{-} h^{+}$pairs in $\mathrm{Ge}, \mathrm{Si}$, and $\mathrm{GaAs}$, obtained using Eq. (4.9) and the radiative absorption coefficients presented in Refs. [125-127]. We assume zero doping and room temperature. 


\section{Trap-assisted recombination}

Impurities in the material lead to energy levels located in between the valence and conduction bands. Such levels are referred to as traps, as they can capture electrons from the conduction band or holes from the valence band. We show a schematic representation of the trapping of an electron in Fig. 12. Differently from band-to-band recombination where an $e^{-} h^{+}$pair is eliminated, here only an electron or a hole disappears by going into the trap.

Trap-assisted recombination is described by the Shockley-Read-Hall (SRH) theory [163]. In the SRH theory, the trap-assisted recombination-rate density is

$$
\begin{aligned}
& \Gamma_{e}^{\text {trap }}=c_{e} n_{t}\left[\left(1-f_{t}\right) n_{e}-f_{t} n_{e t}\right], \\
& \Gamma_{h}^{\text {trap }}=c_{h} n_{t}\left[f_{t} n_{h}-\left(1-f_{t}\right) n_{h t}\right],
\end{aligned}
$$

where $n_{t}$ is the density of impurities leading to traps, $c_{e, h}$ are trapping coefficients (discussed below), and $f_{t}$ is the probability that a trap is occupied by an electron. In Eq. (4.11), we assume that the material is nondegenerate (see Ref. [163] for a discussion of degenerate systems), so that $f_{t}$ is described by Boltzmann statistics:

$$
f_{t}=\exp \left[-\left(E_{t}-E_{F}^{t}\right) / T\right],
$$

where $E_{t}$ is the trap energy level and $E_{F}^{t}$ is the Fermi level or chemical potential of the trap, which sets its occupation probability. In Eq. (4.11), the quantities $n_{e t}$ and $n_{h t}$ are defined, respectively, as

$$
\begin{aligned}
n_{e t} & \equiv N_{C} \exp \left[\left(E_{t}-E_{C}\right) / T\right], \\
n_{h t} & \equiv N_{V} \exp \left[\left(E_{V}-E_{t}\right) / T\right],
\end{aligned}
$$

where $N_{C, V}$ are the conduction and valence band density of states given in Eq. (A2) in Appendix A and Table III and $E_{C, V}$ are the conduction and valence band energies. Note that the rates in Eqs. (4.11) have terms proportional to $n_{e} n_{t}$ or $n_{h} n_{t}$, indicating that trap-assisted recombination requires only a trap and an electron or a trap and a hole. The second terms on the right-hand sides of Eqs. (4.11) ensure that the recombination rates vanish in thermal equilibrium, where the chemical potential of the trap is equal to the equilibrium Fermi level of the semiconductor, whose determination is discussed in Appendix A.

Traps are divided in two types: deep and shallow. Deep traps are those for which the energy levels are well separated from both the conduction and valence bands. They are usually associated with metallic impurities, such as gold, zinc, and copper. Shallow traps, on the other hand, are close to either the valence or conduction band and can be due to dopants such as phosphorus ( $n$-type) and boron ( $p$-type). A summary of the energy levels of different traps for $\mathrm{Si}, \mathrm{Ge}$, and GaAs can be found in Ref. [164].

Trap-assisted recombination can be either radiative (accompanied by photon emission) or nonradiative (by phonon emission). For deep traps, recombination in semiconductors happens usually nonradiatively via multiphonon processes [149,165-167]. Nonradiative deeptrap coefficients are typically of the order of $c_{e, h}^{\text {deep }}=$ $10^{-6}-10^{-9} \mathrm{~cm}^{3} / \mathrm{s}$ [149]. Tables of radiative recombination coefficients by shallow traps can be found in Refs. [149,168]. They are typically of the order of $c_{e, h}^{\text {shallow }}=10^{-13} \mathrm{~cm}^{3} / \mathrm{s}$, much smaller than the ones for deep traps. Thus, for intrinsic semiconductors, trap-assisted recombination happens mostly via deep traps and nonradiatively. Moreover, radiative trap-assisted recombination dominates over its nonradiative counterpart in large-bandgap materials such as phosphors, where it is hard to dispose the large amount of band-gap energy as phonons [149].

The timescale for trap-assisted recombination is

$$
\tau_{e, h}^{\text {trap }}=\frac{n_{e, h}}{\Gamma^{\text {trap }}} .
$$

Since $\Gamma^{\text {trap }}$ is proportional to $n_{h}$ or $n_{e}$, the timescale is independent of the electron or hole number density and is set instead by the trap density. Thus, as electrons and holes diffuse or drift, the trap-assisted recombination timescale stays constant.

\section{Auger recombination}

Finally, electrons and holes can recombine due to the Auger process, in which an electron in the conduction band gives its energy to another carrier by scattering and recombines with a hole, or a hole scatters with another hole and recombines with an electron. In all cases, the process is three-body and leads to the disappearance of both an electron and a hole. The extra carrier in the process dissipates the energy liberated in the recombination in the form of phonons [166]. The corresponding rate density is given by

$$
\Gamma^{\text {Aug }}=\left(a_{n} n_{e}+a_{h} n_{h}\right)\left(n_{e} n_{h}-\bar{n}_{e} \bar{n}_{h}\right),
$$

where $a_{e, h}$ are the Auger recombination coefficients, which are given in Table I for our benchmark semiconductors, and $\bar{n}_{e, h}$ are the background carrier concentrations discussed in Appendix A. The timescale for the Auger process is given by

$$
\tau_{e, h}^{\text {Auger }} \equiv \frac{n_{e, h}}{\Gamma^{\text {Auger }}} .
$$

\section{Other recombination channels}

The processes discussed above are the basic mechanisms by which electrons and holes can recombine. Different combinations of the above processes, or slight variations of them, can lead to more complex recombination channels. Consider, for example, scintillation in GaAs. As discussed 
above, in intrinsic GaAs band-to-band radiative recombination happens efficiently, as GaAs is a direct-band-gap semiconductor. If, for instance, $n$ doping is added, a new radiative recombination channel opens up, as now a hole from the valence band can recombine with an electron provided by the donor by emitting a photon. Doping can significantly increase the GaAs scintillation rate, as the rate is proportional to the number of electrons and holes available to recombine; cf. Eq. (4.8). If the complexity is further increased by also adding $p$ doping to GaAs, which is the case studied in Ref. [92], then further recombination channels become available. For instance, an electron from the donor energy level can then recombine radiatively with a hole in the acceptor energy level. Also, an electron in the conduction band can first transition to the donor energy level and subsequently recombine with a hole in the acceptor level or valence band by emission of a photon [92]. Given the similarity of these processes with the ones already discussed in the previous paragraphs, their analysis can be performed by including additional recombination channels in Eq. (4.2) similar to the ones already presented.

\section{B. Recombination of $e^{-} h^{+}$pairs as a background to dark-matter searches}

Recombination of $e^{-} h^{+}$pairs created by tracks passing through detector materials is an important source of backgrounds at sub-GeV dark-matter experiments. One reason for the relevance of this process is that the energy liberated in the recombination of a single $e^{-} h^{+}$pair is of the order of the material's band gap, i.e., a few eV or less. Such energies coincide with the ones obtained from sub-GeV dark-matter scattering in detectors, so recombination photons or phonons naturally fall within the signal regions of dark-matter searches. In addition, luminescence or phonon events from recombination can avoid track-related vetoes and effectively constitute backgrounds in a variety of ways. As an example, and as discussed in Sec. II B, photons may avoid vetoes if they are long-lived in the material, a possibility that is very natural in band-to-band recombination, since the emitted photons are near band gap. Another possibility, which for brevity is not discussed here in much detail, is that vetoes based on the time coincidence of the luminescence with the originating tracks can be avoided if the return of the excited electrons to the ground state happens slowly, a phenomenon called long-lasting phosphorescence or "afterglow" [169]. Phonons from nonradiative recombination, on the other hand, may avoid vetoes if they are generated in uninstrumented detector materials that are in contact with the detector target, such as holders and clamps, and subsequently make it into the target.

To illustrate how recombination can lead to large backgrounds rates at dark-matter experiments, we discuss the recombination of $e^{-} h^{+}$pairs created by the passage of a single-electron track through a typical detector material. For brevity, we focus here on possible backgrounds from radiative recombination only, and we briefly comment on the relevance of nonradiative recombination in Sec. VID.

We consider the case of a track passing through $\mathrm{Si}$ and take the track energy $E=200 \mathrm{keV}$. According to Fig. 6, such a track travels $\ell_{e} \sim 200 \mu \mathrm{m}$ in Si before stopping. Given the mean ionization energy in room-temperature $\mathrm{Si}$, $\epsilon=3.63 \mathrm{eV}$ [144], the track leads to

$$
N_{e h}=6 \times 10^{4}
$$

$e^{-} h^{+}$pairs distributed as a cloud around the track trajectory (a similar number of $e^{-} h^{+}$pairs is obtained at other temperatures). Assuming that the track is approximately straight, the shape of the cloud is cylindrical. As discussed in the previous sections, the radius of the cylinder $r$ is expected to be a few times the electron and hole mean-free paths, which in $\mathrm{Si}$ are of the order of

$$
r \sim 10^{-2} \mu \mathrm{m}
$$

For our estimates below, in what follows we take the cylinder radius to be equal to Eq. (4.18). The corresponding density of the $e^{-} h^{+}$cloud left by the track is then

$$
n_{e}=n_{h}=\frac{N_{e h}}{\pi r^{2} \ell_{e}} \sim 10^{18} \mathrm{~cm}^{-3} .
$$

After the passage of the track, the electrons and holes in the cloud move due to diffusion or are drifted by an electric field. They also recombine, leading to low-energy photons that contribute to backgrounds. We now calculate the number of recombination photons emitted in the process, first for a track passing through high-purity $\mathrm{Si}$ and later for doped $\mathrm{Si}$.

\section{Passage of a track through undoped $\mathrm{Si}$}

For the case of undoped $\mathrm{Si}$, most of the carriers found in the material are simply those left by the track. In order to understand the fate of these electrons and holes, we must compare the timescales for diffusion, drift, and different recombination mechanisms. Using Eqs. (4.7), (4.10), (4.14), and (4.16), typical initial diffusion and recombination times are

$$
\begin{aligned}
\tau_{0}^{\text {drift }} & \sim \frac{r}{\mu E}=10^{-12} \mathrm{~s}\left[\frac{10^{3} \mathrm{~cm}^{2} / \mathrm{V} / \mathrm{s}}{\mu}\right]\left[\frac{10^{3} \mathrm{~V} / \mathrm{cm}}{E}\right], \\
\tau_{0}^{\text {diff }} & \sim \frac{r^{2}}{D}=10^{-13} \mathrm{~s}\left[\frac{10 \mathrm{~cm}^{2} / \mathrm{s}}{D}\right], \\
\tau_{0}^{\text {direct }} & =\frac{1}{B n_{e, h}}=10^{-4} \mathrm{~s}\left[\frac{10^{-14} \mathrm{~cm}^{3} / \mathrm{s}}{B}\right], \\
\tau_{0}^{\text {trap }} & =\frac{1}{c^{\text {deep }} n_{t}^{\text {deep }}}=10^{-6} \mathrm{~s}\left[\frac{10^{-9} \mathrm{~cm}^{3} / \mathrm{s}}{c^{\text {deep }}}\right]\left[\frac{10^{15} \mathrm{~cm}^{-3}}{n_{t}^{\text {deep }}}\right], \\
\tau_{0}^{\text {Auger }} & =\frac{1}{a n_{e}^{2}}=10^{-5} \mathrm{~s}\left[\frac{10^{-31} \mathrm{~cm}^{6} / \mathrm{s}}{a}\right],
\end{aligned}
$$


where the subscript "0" emphasizes that these are the initial timescales right after the passage of the charged track; we discuss the evolution of the timescales below. In Eq. (4.20), we take typical room-temperature values for the diffusion constant and recombination coefficients from Eq. (4.5) and Table I. In order to obtain the electric drift time, we assume an electric field value $E$ similar to the one applied across the SENSEI CCD to drift electrons and holes toward the readout stage, which is also within a few orders of magnitude of the electric field applied at the SuperCDMS HVeV detector target to induce the Neganov-Trofimov-Luke effect $[68,71]$. We also take a deep-trap density of $n_{t}^{\text {deep }}=10^{15} \mathrm{~cm}^{-3}$ and a trap recombination coefficient of $c^{\text {deep }}=10^{-9} \mathrm{~cm}^{3} / \mathrm{s}$, which are typical values in Si [154]. However, in ultrapure Si, the trap-assisted recombination can be as large as approximately $10^{-2}$ s. [170] Inspecting the timescales in Eq. (4.20), it is clear that, immediately after the passage of the track, the fastest process in action is $e^{-} h^{+}$diffusion. If the electric field is present, the second-fastest process is movement of the pairs by electric drift. After carrier movements, the fastest process is trap-assisted recombination, which, as discussed previously, happens nonradiatively.

Now, as the electrons diffuse radially away from the track trajectory, both the density and the density gradients of the electrons and holes decrease. Correspondingly, the diffusion time increases as the inverse density $\tau^{\text {diff }} \sim 1 / n_{e, h}$, starting from its initial value in Eq. (4.20). The trap-assisted recombination time, however, is independent of the $e^{-} h^{+}$ density and stays constant.

From the above discussion, a clear picture of the evolution of the $e^{-} h^{+}$cloud arises. Consider first the case of the absence of an electric field. After the passage of the track, electron holes quickly diffuse away from the track. The electron and hole densities and density gradients drop, so diffusion slows down until the diffusion timescale becomes comparable to the trap-assisted recombination time: $\tau^{\text {diff }} \sim \tau^{\text {trap }} \sim 10^{-6} \mathrm{~s}$. Finally, after a few trap-assisted recombination times, most of the electron holes disappear by recombining nonradiatively. On the other hand, if an homogeneous electric field across the material is present, carriers first diffuse and then are drifted to the surfaces of the material. If, for instance, an electronics readout stage or electronics connections to ground are located at the surfaces, the carriers leave the material. Otherwise, they eventually recombine close to the surfaces of the material into phonons.

Even if most of the electrons and holes recombine into phonons, a small fraction of them recombine radiatively. The radiative recombination rate, which is proportional to the density of electrons times the one of holes [cf. Eq. (4.8)], is large right after the passage of the track, when these densities are the highest. As electrons and holes diffuse or drift, the radiative recombination rate decreases. Thus, we can obtain an estimate of the number of pairs that recombine radiatively by considering only the process over the first diffusion time. The total number of radiated photons is then

$$
\begin{aligned}
N_{\gamma} & \sim N_{e h} \frac{\tau^{\text {diff }}}{\tau^{\text {direct }}} \\
& \sim 6 \times 10^{-5}\left[\frac{10 \mathrm{~cm}^{2} / \mathrm{s}}{D}\right]\left[\frac{B}{10^{-14} \mathrm{~cm}^{3} / \mathrm{s}}\right] .
\end{aligned}
$$

Comparing the estimate in Eq. (4.21) with the amount of photons created via the Cherenkov effect by a single track [Eq. (2.10)], we find that radiative recombination is a subleading source of photons in undoped Si. However, three important observations elucidate the relevance of radiative recombination. First, while the overall number of recombination photons from a single track is small, all these photons are near band gap (cf. Fig. 13) and, therefore, long-lived, so they can deposit their energy in the detector far from their originating track. As already discussed in Sec. II B, such photons may efficiently constitute backgrounds in, e.g., CCD-based detectors, since they can avoid track-related vetoes. Second, while the rate for radiative recombination is small in high-purity $\mathrm{Si}$, the situation is drastically different in the doped scenario, which we discuss below. Third, the radiative recombination coefficient is significantly enhanced at cryogenic temperatures [161], and in high-purity semiconductors trap-assisted phononmediated recombination is suppressed. In these setups, radiative recombination can be a significant source of backgrounds. The enhancement of the radiative recombination coefficient at low temperatures is particularly relevant, as many dark-matter detectors are, in fact, operated cryogenically.

\section{Passage of a track through doped Si}

When doping is included, in addition to the $e^{-} h^{+}$pairs created from the passage of the track, a large background density of electrons or holes from the dopant already exists in the material. This enhances radiative recombination of the pairs left by the track, as these electrons or holes can recombine with the large number of background carriers donated by the dopant.

For concreteness, let us consider the case of $n$-doped $\mathrm{Si}$ with an $n$-doping density $n_{d}=10^{18} \mathrm{~cm}^{-3}$. In this case, holes left by the track recombine with the background electrons from the dopant, and the timescales for the corresponding hole recombination channels are

$$
\begin{aligned}
\tau^{\text {direct }} & =\frac{1}{B n_{e}}=10^{-4} \mathrm{~s}\left[\frac{10^{-14} \mathrm{~cm}^{3} / \mathrm{s}}{B}\right], \\
\tau^{\text {trap }} & =\frac{1}{c^{\text {deep }} n_{t}^{\text {deep }}}=10^{-6} \mathrm{~s}\left[\frac{10^{-9} \mathrm{~cm}^{3} / \mathrm{s}}{c^{\text {deep }}}\right]\left[\frac{10^{15} \mathrm{~cm}^{-3}}{n_{t}^{\text {deep }}}\right], \\
\tau^{\text {Auger }} & =\frac{1}{a n_{e}^{2}}=10^{-5} \mathrm{~s}\left[\frac{10^{-31} \mathrm{~cm}^{6} / \mathrm{s}}{a}\right] .
\end{aligned}
$$


Differently from the undoped scenario, these timescales do not evolve in time, as they depend on only the electron density, which is set by the dopant and not affected by the diffusion of the holes left by the track. As a consequence, we can obtain the total number of recombination photons by simply multiplying the radiative recombination rate with the lifetime of the holes, which is set by the fastest recombination process in Eq. (4.22), i.e., by trap-assisted recombination. The total number of photons obtained from recombination for our single-electron track is then given by

$$
\begin{aligned}
& N_{\gamma} \sim N_{e h} \frac{\tau^{\text {trap }}}{\tau^{\text {direct }}} \\
& \sim 6 \times 10^{2}\left[\frac{10^{-9} \mathrm{~cm}^{3} / \mathrm{s}}{c^{\text {deep }}}\right]\left[\frac{10^{15} \mathrm{~cm}^{-3}}{n_{t}^{\text {deep }}}\right] \\
& \times\left[\frac{B}{10^{-14} \mathrm{~cm}^{3} / \mathrm{s}}\right]
\end{aligned}
$$

The large number of photons obtained in our estimate clearly shows the relevance of radiative recombination as a source of backgrounds in dark-matter detectors. Note that this number is also many orders of magnitude larger than the one in Eq. (4.21) obtained for undoped $\mathrm{Si}$ and also larger than the number of photons with approximately $1-\mathrm{eV}$ energies obtained from the Cherenkov process by the same track [Eq. (2.10)]. In the next section, we demonstrate that radiative recombination in SENSEI is an important background, since the CCD back side is unthinned and has a few-micron-thick layer with high phosphorus doping.

\section{RADIATIVE BACKGROUNDS AT CURRENT DARK-MATTER EXPERIMENTS}

In this section, we give an overview of how backgrounds from Cherenkov radiation, recombination, and transition radiation are realized in recent results from SENSEI at MINOS [68], SuperCDMS HVeV [71], and EDELWEISS [70]. We also briefly discuss the results from CRESST-III $[61,62]$, the EDELWEISS-Surf Ge bolometer [48], and the SuperCDMS cryogenic photodetector (CPD) [72]. In Sec. VI, we discuss upcoming and proposed experiments.

Our selection of experiments is representative of a variety of detection strategies and environmental characteristics. For example, the data from SENSEI at MINOS and SuperCDMS-HVeV are taken at a shallow underground site or on the surface, respectively, with little shielding [68,71], while the data from EDELWEISS are taken deep underground in a well-shielded environment [70]. All experiments have excellent timing resolution with the exception of SENSEI, which instead has excellent position resolution and very little timing information. Detectors with good timing resolution are not affected by radiative backgrounds that are generated in the target itself, since such photons are detected at the same time as the high-energy charged track. For these detectors, radiative backgrounds are observed as low-energy events if they originate from uninstrumented materials near the target. At SENSEI and other CCD-based detectors, on the other hand, background photons can be generated in both the target and surrounding material.

We see that a precise calculation of the Cherenkov radiation, recombination, and transition radiation backgrounds is, in general, rather complicated. However, our discussion makes it clear that these processes undoubtably contribute to the low-energy backgrounds at current and future experiments.

\section{A. SENSEI at MINOS}

The SENSEI experiment uses Si Skipper chargedcoupled devices to probe dark matter that interacts with electrons [22,35,46,68]. Dark-matter scattering in an approximately 1.9 g Skipper CCD excites electrons from the $\mathrm{Si}$ valence band into the conduction band, creating $e^{-} h^{+}$pairs. The excited pairs are quickly drifted to the Skipper CCD's surface via an applied electric field, where they are stored until being read out.

We focus on the results reported by SENSEI in Ref. [68]. These results are based on data collected during 24 days in early 2020 at the MINOS cavern at Fermilab. During the 24 days, the Skipper CCD is exposed 22 times, for a period of $20 \mathrm{~h}$ each time, which is followed by a 5.15-h reading period. Each such $20-\mathrm{h}$ exposure plus readout is called a "CCD image." The Skipper CCD has a thickness of $675 \mu \mathrm{m}$ and a total area of size approximately $9.4 \mathrm{~cm} \times 1.6 \mathrm{~cm}$. The latter area is divided into a two-dimensional array of pixels of size $15 \mu \mathrm{m} \times 15 \mu \mathrm{m}$, with each pixel having the same thickness as the CCD. Signal events are categorized by the number of $e^{-} h^{+}$pairs that they create within sets of pixels defined by the search analysis ( $1 e^{-}$events, $2 e^{-}$events, etc.).

To mitigate backgrounds arising from high-energy events such as charged tracks, SENSEI imposes several selection criteria ("cuts") on the data. One of the most important cuts is the "halo mask." High-energy events leave tracks of energy depositions in the CCD, in the form of multiple $e^{-} h^{+}$pairs along the track. To discriminate such background events from the hypothetical dark-matter signal, all the pixels that have a distance to a track smaller than some "halo-mask radius" (measured in number of pixels) are removed from the analysis. For quoting a final single-electron-event rate, a benchmark halo-mask radius of 60 pixels is chosen, outside of which the rate of singleelectron events is reported to be $R_{1 e^{-}} \sim(450 \pm 45) /$ g-days. Several hypotheses to explain these events are discussed in Ref. [68], but no currently known background is able to account for them.

Here, we propose two novel explanations for the origin of the single-electron events: We argue that a significant fraction of them is due to the emission of (i) Cherenkov and (ii) recombination photons that are generated as charged particles pass through the detector. [171] Transition radiation also contributes single-electron events, but the corresponding 

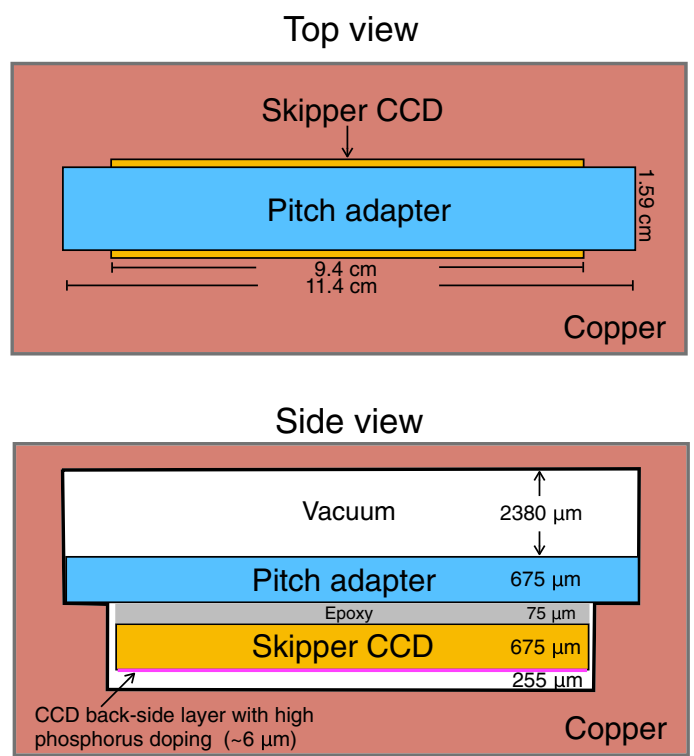

FIG. 14. Schematic depiction of the SENSEI Skipper CCD detector module in the MINOS cavern, a shallow underground site, for taking the data described in Ref. [68]. The active part of the detector is the Skipper CCD (orange). A Si pitch adapter (blue) is glued with epoxy (gray) to the Skipper CCD. This is placed inside a copper module (red). The CCD back side contains a few-micron-thick layer that is heavily doped with phosphorus (magenta). We omit several details of the detector, including a series of layers with thicknesses of the order of approximately $1 \mu \mathrm{m}$ or below that exist in between the Skipper CCD and the epoxy. The entire copper module shown is surrounded by lead bricks, placed inside a copper vessel, and then surrounded with additional lead shielding.

rate at SENSEI is negligible compared to the rates obtained from Cherenkov and recombination photons. [172].

We present a simplified schematic of the SENSEI detector in Fig. 14. The main parts of the detector are the CCD and a "pitch adapter," both made of $\mathrm{Si}$, and the epoxy glue between them. The back side of the CCD contains a $\mathcal{O}$ (few-micron)-thick layer of $\mathrm{Si}$ that is highly doped with phosphorus. These parts are placed in a copper housing.

\section{Cherenkov photons}

Cherenkov photons are emitted by tracks as they pass through the CCD, pitch adapter, or epoxy glue. For brevity, we consider here only Cherenkov radiation arising from the $\mathrm{CCD}$, which, given its dimensions compared with the pitch and epoxy glue, should already provide an $\mathcal{O}(1)$ fraction of all Cherenkov photons.

In order for the Cherenkov photons to be registered as events after applying the halo-mask cut, they must travel at least 60 pixels $(900 \mu \mathrm{m})$ away from their originating track before converting into $e^{-} h^{+}$pairs. As discussed in Sec. II A, photons have a large mean-free path in the dielectric material if they have energies near or below its

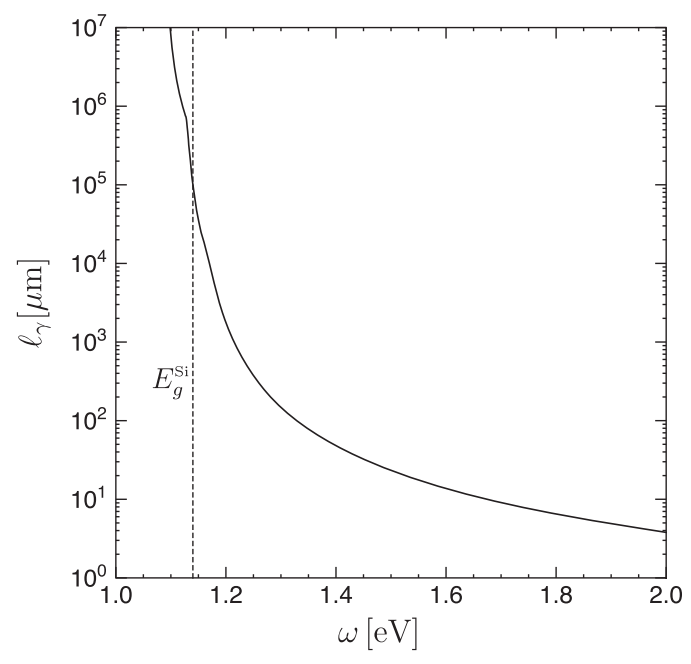

FIG. 15. Photon attenuation length in $\mathrm{Si}$ for energies close to the indirect $\mathrm{Si}$ band gap, $E_{g}^{\mathrm{Si}}=1.14 \mathrm{eV}$, at the SENSEI operating temperature $T=135 \mathrm{~K}$. The data are taken from a fit in Ref. [173], which allows for single-phonon-assisted transitions.

band gap. In Fig. 15, we show the photon mean-free path in $\mathrm{Si}$ at the SENSEI operating temperature $T=135 \mathrm{~K}$. From the figure, we see that only photons with energy $\omega \lesssim 1.2 \mathrm{eV}$ can travel more than 60 pixels $=900 \mu \mathrm{m}$ radially away from the track. On the other hand, only photons with frequency above $\omega \gtrsim 1.1 \mathrm{eV}$ are energetic enough to create $e^{-} h^{+}$pairs in Si [173]. Thus, we can obtain an order-of-magnitude estimate of the number of Cherenkov events by calculating the total number of Cherenkov photons in the frequency range $1.1 \leq \omega \leq 1.2 \mathrm{eV}$, which are emitted by all the charged tracks passing through the Skipper CCD. The charged tracks and their energies are measured by SENSEI and are reported in Ref. [68]. With this procedure, we find that the number of single-electron events expected at SENSEI from Cherenkov backgrounds is (see Appendix B for more details)

$$
R_{1 e^{-}}^{\text {Cherenkov }} \sim 500 / \text { g-day. }
$$

This compares favorably with the observed rate $R_{1 e^{-}}=$ $450 / \mathrm{g}$-day, although, as we discuss below, this result should be seen as only a rough estimate.

Further evidence in favor of our hypothesis comes from analyzing the Cherenkov event rate as a function of the halo-mask radius. Besides the benchmark halo radius of 60 pixels, SENSEI also reports rates for other halo-mask radii. They find that the single-electron-event rate increases for smaller halo-mask radii, i.e., when including events closer to the tracks. As an example, when reducing the halo radius from its benchmark value to approximately five pixels, the single-electron-event rate is measured to increase to approximately 900/g-days. Our hypothesis also accounts for the increase of the rate as the halo mask is decreased, since most of the emitted Cherenkov photons 


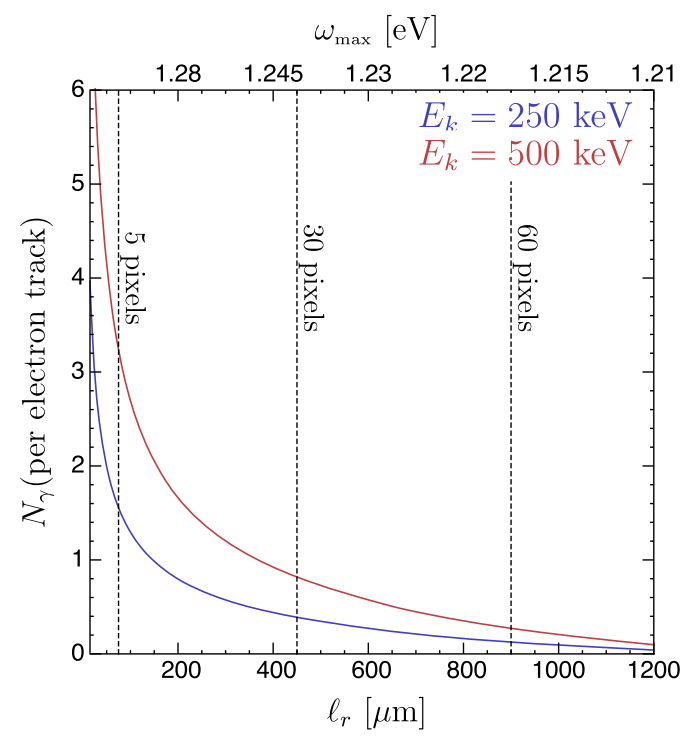

FIG. 16. Total number of photons produced by a single highenergy electron track passing through $\mathrm{Si}$, which travel radially away from the track for a distance of at least $\ell_{r}$. Such photons have a mean-free path satisfying $\ell_{\gamma} \geq \ell_{r} / \sin \theta_{\mathrm{Ch}}$. The electron's kinetic energy is $E_{k}=250 \mathrm{keV}$ (blue curve) or $E_{k}=500 \mathrm{keV}$ (red curve). The top $x$ axis shows the maximum frequency that allows the photon to travel at least $\ell_{r}$. Vertical lines show the dimensions corresponding to 5, 30, and 60 pixels in the Skipper CCD at SENSEI.

are converted into $e^{-} h^{+}$pairs close to their originating track. This is illustrated in Fig. 16, where we show the number of Cherenkov photons that arise from one electron track and that travel at least a distance $\ell_{r}$ radially away from the track before converting into an $e^{-} h^{+}$pair. Such photons, thus, pass the cut corresponding to a halo-mask radius of the order of $\ell_{r}$. From the figure, we clearly observe that the number of expected Cherenkov-induced $e^{-} h^{+}$events increases as smaller halo-mask radii are considered.

\section{Luminescence from recombination of $e^{-} h^{+}$pairs}

A second possible contribution to the backgrounds observed by SENSEI are photons that arise from the recombination of $e^{-} h^{+}$pairs, which are created as charged tracks pass through the CCD, epoxy, or pitch adapter. As discussed in Sec. IV B, each track generates tens of thousands of $e^{-} h^{+}$pairs when passing through these materials, which can recombine either radiatively or nonradiatively. The rates for the different recombination mechanisms are material dependent. The CCD and pitch adapter found in SENSEI are made mostly out of highpurity, undoped Si. In addition, while there is no electric field in the pitch adapter, there is a nonzero electric field across the bulk of the CCD. In both cases, we know from Sec. IV B that only a tiny fraction of the $e^{-} h^{+}$pairs recombine radiatively [cf. Eq. (4.21)], naively suggesting that recombination is not a relevant source of backgrounds for SENSEI.

Very importantly, however, the back side of SENSEI's CCD (as seen in Fig. 14) has a few-micron-thin layer of phosphorus-doped $\mathrm{Si}$, where the doping concentration is as high as $10^{20} \mathrm{~cm}^{-3}$ and where there is no electric field. Even if this region is small, in doped materials radiative recombination is extremely efficient, as discussed in Sec. IV B. As a consequence, as tracks pass through the doped layers, they lead to a large number of recombination photons, which can travel to and be absorbed in the bulk of the CCD and contribute to the observed single-electron events. Moreover, most of the recombination photons are near band gap and, thus, have a large absorption length, so they can avoid the halo-mask cut.

As with the Cherenkov events, to estimate the number of single-electron events now due to recombination, we calculate the total number of photons emitted in the range of frequencies $1.1 \mathrm{eV} \leq \omega \leq 1.2 \mathrm{eV}$. Using the radiative recombination fraction in Eq. (4.23), the spectrum Eq. (4.9), and the number of tracks observed at SENSEI, we obtain (for details, see Appendix B)

$$
R_{1 e^{-}}^{\text {recombination }} \sim 500 / \text { g-day. }
$$

Our estimate clearly indicates that radiative recombination in the doped layers of the CCD is an important source of backgrounds at SENSEI and may explain a large fraction of the observed single-electron-event rate.

Finally, we comment briefly on the possible luminescence of epoxy. After the passage of a charged particle through the epoxy, it is possible that some of the deposited energy is reemitted as radiation, which can produce events in the CCD. The front side of the CCD (which faces the epoxy) contains a poly-Si layer with a thickness of about $0.6 \mu \mathrm{m}$. Photons need to travel through this layer and be absorbed in the Si bulk in order to be registered as an event. Only photons with an energy below approximately $2.8 \mathrm{eV}$ can traverse the front-side layer. It is possible that the epoxy luminesces below this energy [174]. It may be possible, even if unlikely [175], that such events occasionally create a (single-pixel) $2 e^{-}$event. A detailed study of the luminosity and scintillation efficiency of epoxy is needed to characterize these possible backgrounds more precisely.

\section{Transition radiation}

Transition radiation from the copper-vacuum, vacuumCCD, vacuum-pitch-adapter, and other interfaces can produce photons across a wide range of energies. [176] However, in order for photons to be registered as an event, they first need to penetrate into the bulk of the $\mathrm{CCD}$, which requires their energy to be close to the band gap. Most of the CCD front side is covered by the pitch adapter and epoxy, requiring photons that are generated at the copper-vacuum interface to be within $0.1 \mathrm{eV}$ of the 
band gap. Even the small regions that are uncovered have a $0.6-\mu \mathrm{m}$-thick poly-Si layer (see the discussion above), requiring photons to have an energy below approximately $2.8 \mathrm{eV}$ to traverse into the bulk. On the back side, there is an approximately 6- $\mu \mathrm{m}$ layer of ( $n$-doped) $\mathrm{Si}$ and poly-Si, requiring the photon energies to be below approximately $1.8 \mathrm{eV}$ in order to be registered in the bulk. We find that transition radiation contributes much less than Cherenkov radiation or recombination to the single-electron-event rates. More details are provided in Appendix B.

\section{Summary}

Summing both our Cherenkov and recombination eventrate estimations, we see that both of these processes can easily generate over 1000 events per g-day, a number that is a factor of approximately 2 larger than the measured rate of 450/g-day. Our calculations, however, must be taken as only order-of-magnitude estimates, as we neglect a myriad of details that must be accounted for to calculate a precise background event rate. In order to refine our estimates, a detailed simulation of these two sources of backgrounds in the SENSEI detector is required, which will be done in a companion paper [178]. In that reference, we will discuss several aspects that are crucial for obtaining a realistic background rate estimate, which include (i) the precise distribution of the electron and muon tracks in the detector, (ii) all data analysis cuts, (iii) the exact geometry and properties of the detector materials, (iv) reflection and transmission of Cherenkov and recombination photons on detector surfaces, (v) the detailed properties of the layers of doped material in the CCD, and (vi) absorption of Cherenkov and recombination photons in the phosphorusdoped CCD layers and the epoxy. There, we confirm that our very rough estimates [Eqs. (5.1) and (5.2)] are within a factor of $\mathcal{O}(1-10)$ of a much more detailed background simulation. In Ref. [178], we will also discuss the $2 e^{-} h^{+}$-pair event rate.

\section{B. SuperCDMS HVeV above ground}

The SuperCDMS high-voltage eV-resolution ( $\mathrm{HVeV})$ detectors $[36,71]$ aim to detect dark matter via its scattering with electrons in a Si target. The $e^{-} h^{+}$pairs created in the scattering process are drifted toward the surfaces of the $\mathrm{Si}$ target by an applied bias voltage, creating phonons along their way via the Neganov-Trofimov-Luke effect $[179,180]$. In this way, the energy deposited by the scattering event is amplified, so that a small energy resolution can be achieved. The phonon energy is measured by quasiparticle-trapassisted electrothermal-feedback transition edge sensors (QETs) attached to the surface of the Si detector.

In our discussion, we focus on the detector setup and results described in Ref. [71,181], which correspond to data collected during 7 days in 2019 in a surface laboratory at Northwestern University using a $0.93 \mathrm{~g} \mathrm{Si}$ crystal target. Given that the detector is near surface, it is exposed to a large number of backgrounds from cosmic high-energy
TABLE II. The rate of $n e^{-} h^{+}$pairs, $R_{n}$, observed with the SuperCDMS HVeV detector for two different bias voltages [71]. The uncertainty on the quoted rates corresponds to $3 \sigma$, assuming Poisson statistics.

\begin{tabular}{lcc}
\hline \hline & \multicolumn{2}{c}{$\mathrm{HVV}$ rates (g-day) } \\
\cline { 2 - 3 } & $100 \mathrm{~V}$ & $60 \mathrm{~V}$ \\
\hline$R_{1}$ & $(149 \pm 1) 10^{3}$ & $(165 \pm 2) 10^{3}$ \\
$R_{2}$ & $(1.1 \pm 0.1) 10^{3}$ & $(1.2 \pm 0.2) 10^{3}$ \\
$R_{3}$ & $207 \pm 40$ & $245 \pm 86$ \\
$R_{4}$ & $53 \pm 20$ & $77 \pm 48$ \\
$R_{5}$ & $16 \pm 11$ & $20 \pm 25$ \\
$R_{6}$ & $5 \pm 6$ & $10 \pm 17$ \\
\hline \hline
\end{tabular}

events. One important tool available at SuperCDMS to discriminate signal events from backgrounds is the detector's excellent timing resolution (of the order of $10 \mu \mathrm{s}$ ), which allows events that coincide with the passage of energetic tracks to be vetoed. More specifically, physics events are identified using a pulse shape, which is of the order of $100 \mu \mathrm{s}$. Note that this is in sharp contrast with the strategy followed by SENSEI discussed previously, where there is little timing information and backgrounds are instead discriminated using information regarding the location of energy depositions in the CCD.

The final dark-matter search dataset observed by SuperCDMS is a spectrum of $n-e^{-} h^{+}$-pair events registered within their pulse-shape time window, where $n$ ranges from 1 to 6 [71]. We show their results in Table II. The observed single- $e^{-} h^{+}$-pair events are likely dominated by charge leakage [183]; however, the origin of the events with $n \geq 2$ $e^{-} h^{+}$pairs is unknown [71]. SuperCDMS tests the results with different bias voltages (100 and $60 \mathrm{~V})$, finding that the rate of events is similar for both voltages.

We now argue that Cherenkov photons generated in the nonconductive material surrounding the $\mathrm{HVeV} \mathrm{Si} \mathrm{detector}$ can plausibly explain the number of events containing $n \geq 2 e^{-} h^{+}$pairs, both in magnitude and in spectral shape. We also mention the contribution from transition radiation, which is, however, likely subdominant compared to that from Cherenkov photons. In addition, luminescence from the materials surrounding the target could contribute to the backgrounds, but nothing precise can be said about this possibility without detailed measurements of the luminescence properties of these materials [184].

A schematic layout of the main components of the $\mathrm{HVeV}$ detector module inside its copper housing is presented in Fig. 17. The $\mathrm{Si} \mathrm{HVeV} \mathrm{detector} \mathrm{is} \mathrm{clamped} \mathrm{with} \mathrm{metallic}$ screws between two PCBs made of a material composed of woven fiberglass cloth with an epoxy resin binder.

\section{Cherenkov photons}

Cherenkov photons are generated by tracks passing through the PCBs, the Si detector, and other dielectrics 

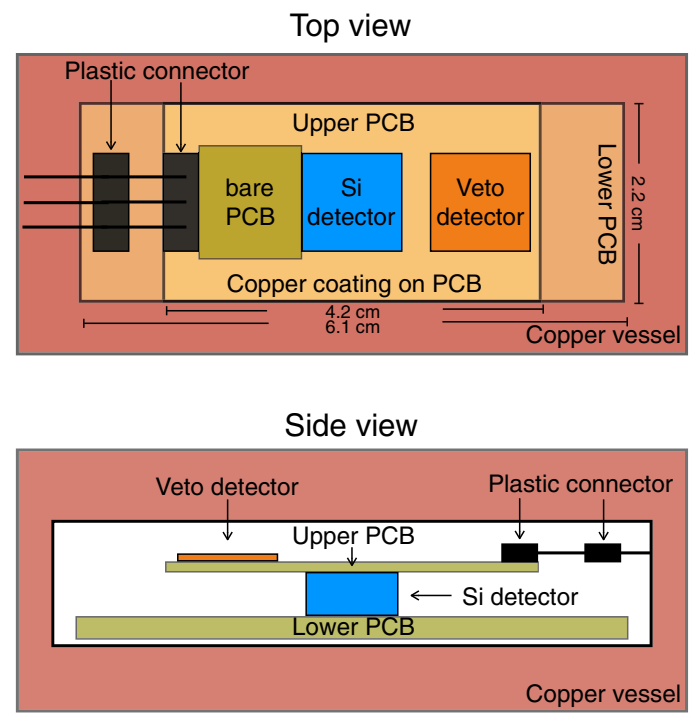

FIG. 17. Schematic depiction of the SuperCDMS HVeV detector module that takes surface data described in Ref. [71]. The active part of the detector is the "Si detector" (blue), which is clamped between two printed-circuit boards (PCBs, gray-green) that are partially covered with a layer of copper (two shades of tan color). All surfaces of the two PCBs are coated with copper, except the area denoted as "bare PCB" on the front surface of the upper PCB from the top view, as well as the side surfaces. A Si "veto detector" is placed on top of the top PCB. Two plastic connectors (black) and a flex cable (not shown) are also located in the copper housing (red).

found in smaller quantities within the copper vessel, such as plastic connectors. Cherenkov photons produced by tracks passing through the target are easily removed by vetoing time windows around the high-energy event, as described above. That leaves us only with the Cherenkov photons due to tracks that pass exclusively through uninstrumented materials, such as the PCBs, which are not vetoed. Such photons may escape the uninstrumented materials and make their way into the detector (either directly or after reflecting one or more times off the copper or other surfaces inside the copper vessel), producing a low-energy background. We now provide an overview on how to estimate the number of photons created in this way, concentrating in photons originating in the PCB. We refer the reader to Appendix $\mathrm{C}$ for a more detailed explanation of our calculations.

The number and spectrum of Cherenkov photons created in the PCB can be obtained given its dielectric properties and the number of tracks going through it, the details of which are not available. We assume that the PCB's dielectric properties are similar to the "NEMA FR4 plate" found in Ref. [185], which is made of $61 \% \mathrm{SiO}_{2}, 15 \%$ epoxy, and $24 \%$ of bromine and oxygen. We then approximate the real part of the dielectric function, $\operatorname{Re} \epsilon(\omega)$, by the PCB's dominant $\mathrm{SiO}_{2}$ component (see Sec. II A). To determine if photons can escape the PCB, we estimate the PCB's photon absorption coefficient from the absorption coefficient of its $\mathrm{SiO}_{2}$ and epoxy components, ignoring possible contributions from bromine and oxygen. We take the photon absorption length in $\mathrm{SiO}_{2}$ from Ref. [114] and epoxy from Ref. [186].

To estimate the number of tracks passing through the $\mathrm{PCB}$, we proceed as follows. For the incident high-energy muon track rate and energy spectrum, we use the data in Ref. [187]. There is no public information available for the rate and spectrum of the high-energy electron background, so we simply assume that the spectrum is the same as that observed at SENSEI [68] but normalized higher by a factor of 60 given that SuperCDMS is closer to the surface. This brings the background rate in line with the one observed in the SuperCDMS cryogenic photodetector (CPD) [72] and should constitute a reasonable estimate of the background rate in the $\mathrm{HVeV}$ detector [183]. In addition, we consider only tracks that pass sufficiently close to the edges of the PCB board, which lead to photons that have a large chance of escaping given the PCB's absorption coefficient. It is very plausible that a large fraction of such tracks pass through the PCB without hitting instrumented parts of the detector and are, thus, not vetoed.

With the above assumptions, we estimate that the number of Cherenkov radiation events per g-day of detector exposure from interactions of charged particles in the PCB (see Appendix C for details) is

$$
N_{\text {events }}^{\text {Cherenkov }} \sim 2.8 \times 10^{4} / \text { g-day. }
$$

Each of these events contains some number of Cherenkov photons with a range of energies, some of which may escape the PCB. Given that the PCB has a frequencydependent absorption coefficient, the photons that can escape the PCB inherit a characteristic spectrum, shown in Fig. 18 for photons originating from a typical (average) track. Some of the Cherenkov photons that exit the PCB are expected to reach the detector target, either directly or after bouncing off various materials or the copper walls of the vessel. As shown in Fig. 18, many of these photons have energies above the Si band gap, so these produce $e^{-} h^{+}$ pairs if they are absorbed in the Si detector. The observed $n$ electron events are then obtained from the absorption of one or multiple Cherenkov photons, depending on the energy of each photon and the charge yield in Si. Given the large number of photons obtained in our estimate as compared with the rates measured at SuperCDMS, it is very plausible that Cherenkov photons are an important source of backgrounds at the $\mathrm{HVeV}$ detector.

In order to calculate the actual rates of $n$-electron events from Cherenkov radiation, it is necessary to know the probability $f$ for a photon that has escaped the PCB to reach the detector target without being absorbed by other detector parts. Calculating this detection probability requires a detailed detector simulation, which is beyond 


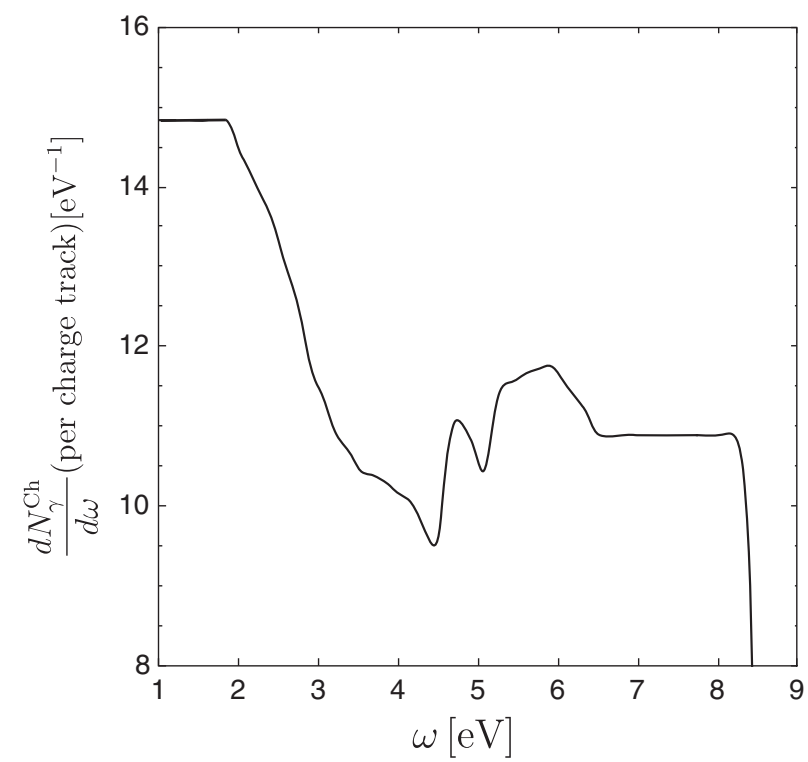

FIG. 18. The spectrum of Cherenkov photons that are able to escape the $\mathrm{PCB}$, produced by an average single charged particle interacting in the PCB. The sharp drop in the spectrum at approximately $8 \mathrm{eV}$ is due to the $\mathrm{SiO}_{2}$ component of the $\mathrm{PCB}$, which becomes highly absorptive at photon frequencies close to and above the material's band gap. See Appendix C 1 for details.

the scope of this work. However, an alternative way to obtain this probability is to simply fit the predicted $n$-electron spectrum for a given $f$ to the observed SuperCDMS data, assuming that all the measured $n \geq 2$ events are due to Cherenkov photons (recall that the singleelectron events are expected to come from charge leakage). With this assumption and ignoring that $f$ is likely energy dependent, we obtain $f \approx 1.6 \times 10^{-3}$. The smallness of the detection probability required to fit the data is indicative of the extremely large number of Cherenkov photons that are being created by tracks at the PCB. With this oneparameter background model, we can predict six observables, namely, the Cherenkov-induced $n$-electron spectrum shape $1 \leq n \leq 6$. We show the corresponding spectrum shape in Fig. 19. The figure clearly indicates that Cherenkov photons coming from the detector's PCB could plausibly precisely reproduce the observed event spectrum of the $n \geq 2$ electron events.

While our hypothesis that Cherenkov radiation explains the observed low-energy events with $n \geq 2 e^{-} h^{+}$pairs is plausible, we cannot definitively prove that all, or even most, of the observed events are from Cherenkov radiation. For this, we need to know (i) the precise spectrum of the high-energy background, (ii) the optical properties of the PCBs and all dielectric materials inside the copper vessel, and (iii) a better understanding of the detection probability $f$ of each Cherenkov photon, etc. This requires a careful measurement of the optical properties of the PCB and the

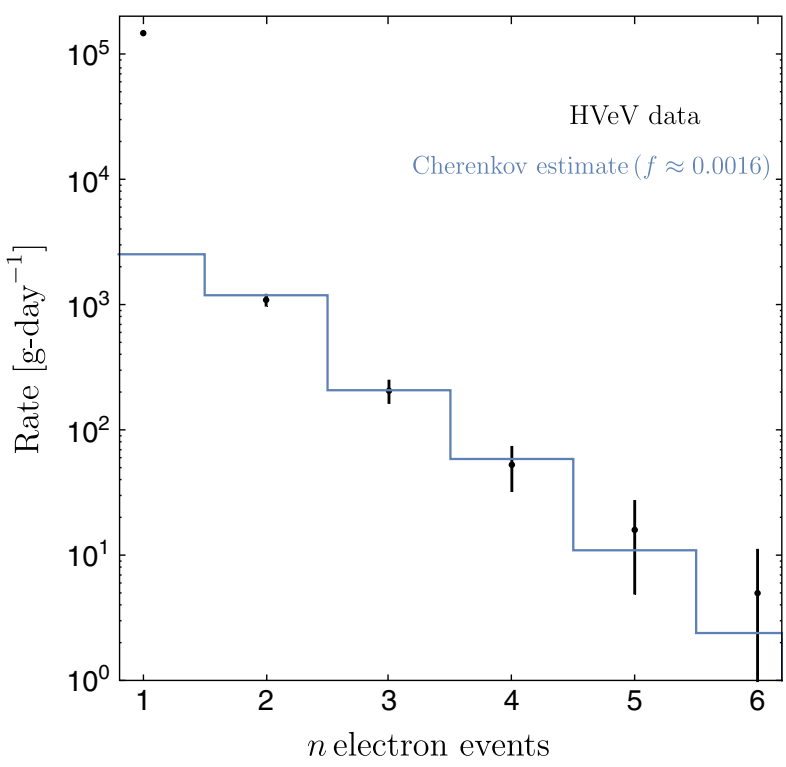

FIG. 19. The estimated $n$-electron event rate from Cherenkov radiation at SuperCDMS HVeV (blue line) based on a simple model with several assumptions and a photon detection probability $f \approx 1.6 \times 10^{-3}$ (see the text for details). The experimental data with $100 \mathrm{~V}$ bias voltage are shown in black with the error bars corresponding to the $3 \sigma$ statistical uncertainty [71].

other nonconductive materials inside the copper housing, as well as a detailed simulation.

\section{Transition radiation}

We now discuss the contribution from transition radiation. When charged particles pass through the surfaces of the copper vessel, PCB, or other materials, without intersecting the instrumented target, they generate photons from transition radiation. These photons can be registered as an event once they reach the detector. Considering only the surfaces with the largest area, i.e., the inner wall of the copper vessel, we estimate that the total number of transition radiation events is

$$
N_{\text {events }}^{\mathrm{TR}} \sim 1000 / \text { g-day. }
$$

More details of our estimate can be found in Appendix C. Comparing this number with $N_{\text {events }}^{\text {Cherenk }}$ in Eq. (5.3) suggests that transition radiation provides a much smaller contribution to the observed event rate. For example, assuming the same detection probability $f$ mentioned above, transition radiation contributes negligibly to $n$ electron events at SuperCDMS. However, since the spectrum of an emitted photon is quite different than that of Cherenkov photons, the detection probability $f$ might not be the same, so we cannot draw a definite conclusion without a dedicated simulation. Moreover, the PCB consists of different materials that are woven together; it is 
possible that a track can make multiple transitions between different materials inside the $\mathrm{PCB}$, thereby generating a large number of transition photons.

The value of $N_{\mathrm{event}}^{\mathrm{TR}}$ in Eq. (5.4) is still very large, so that transition radiation should be carefully studied as a source of low-energy backgrounds in this and future versions of SuperCDMS-HVeV. In particular, transition radiation could be an important background source even in the absence of nonconductive materials in the detector housing (which would remove Cherenkov radiation).

\section{Luminescence}

We are unable to say anything quantitative about possible luminescence backgrounds at SuperCDMS$\mathrm{HVeV}$. It is possible that some low-energy events are generated if the PCB has a nonzero luminescence, but this requires detailed measurements of its properties. That being said, it is very important to point out that radiative recombination is expected to be significantly enhanced at cryogenic temperatures (see, e.g., Ref. [161] for the case of recombination in $\mathrm{Si}$ at low temperatures), so it could be a significant source of low-energy photons.

\section{EDELWEISS at Modane}

The EDELWEISS Collaboration published a first search for sub-GeV dark matter using a Ge cryogenic target in Ref. [70]. In this search, electrons produced in the Ge target are drifted with a bias voltage (approximately $78 \mathrm{~V}$ ) and amplified, creating phonons via the Luke-NeganovTrofimov effect $[179,180]$. While the detection principle is similar to the SuperCDMS HVeV search discussed in the previous section, an important difference is that the EDELWEISS Ge detector is operated in a well-shielded, underground, low-background environment at the Modane Underground Laboratory. Nevertheless, a large event rate is observed, peaking at approximately $4 \times 10^{8}$ differentialrate units [188] (DRU) at an ionization energy equal to approximately $4 \mathrm{eV}$ and flattening out at a rate of approximately $10^{5}$ DRU for ionization energies in the range approximately $15 \mathrm{eV}$. The total exposure is approximately 2.4 days. We see below that, given the quiet environment in which the detector is placed, such events cannot originate from high-energy tracks going through the materials near the target sensor. This is regardless of the mechanism by which the charged tracks produce radiation, may it be Cherenkov radiation, transition radiation, or luminescence. However, we also show that Cherenkov backgrounds, in particular, and perhaps even luminescence due to $e^{-} h^{+}$pairs created by tracks, could still be large enough to limit the future sensitivity of this experiment to sub-GeV dark matter. Transition radiation constitutes likely only a small background, since many of the tracks that pass, e.g., the copper-vacuum interface also hit the detector and are vetoed.

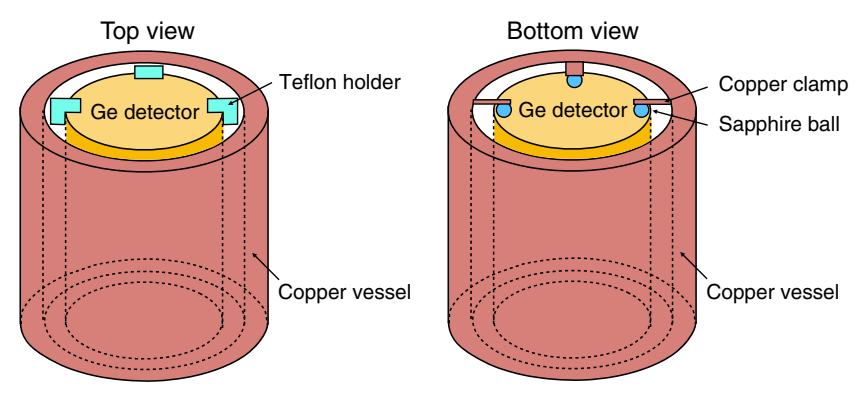

FIG. 20. Schematic depiction of the EDELWEISS Ge detector module used to take the data described in Ref. [70]. The Teflon holders each have a mass of $0.05 \mathrm{~g}\left(\rho_{\text {PTFE }}=2.2 \mathrm{~g} / \mathrm{cm}^{3}\right)$. The sapphire balls have a diameter of $3.18 \mathrm{~mm}$, and each has a mass of $0.067 \mathrm{~g}\left(\rho_{\mathrm{Al}_{2} \mathrm{O}_{3}}=3.98 \mathrm{~g} / \mathrm{cm}^{3}\right)$.

A schematic of the EDELWEISS detector is given in Fig. 20. It consists of a $33.4 \mathrm{~g}$ Ge target that is contained inside a copper housing. The detector is clamped between three Teflon (PTFE) holders on the one side and three sapphire spheres on the other side. High-energy tracks that interact with either the uninstrumented Teflon holders or sapphire spheres can produce radiation. It is possible that the heat produced by the high-energy events that interact in the Teflon is detected also in the EDELWEISS detector [189], so that radiation produced in the Teflon could be vetoed; however, this does not seem to be possible with the events produced in the sapphire.

Given the deep underground location of the detector, cosmic high-energy events are rare, so the tracks passing through the sapphire or Teflon are mostly due to radioactivity intrinsic to these materials or from environmental radioactivity. The most important source of radioactive backgrounds is the former, which is due to the contaminants ${ }^{238} \mathrm{U}$ and ${ }^{40} \mathrm{~K}$ [190]. The Teflon holders have a measured ${ }^{238} \mathrm{U}$ contamination of $10 \pm 5 \mathrm{mBq} / \mathrm{kg}$, while their ${ }^{40} \mathrm{~K}$ contamination is not reported. The contamination of the sapphire spheres is unknown. Assuming that the ${ }^{238} \mathrm{U}$ and ${ }^{40} \mathrm{~K}$ contamination of the Teflon and sapphire is all equal at a rate of $10 \mathrm{mBq} / \mathrm{kg}$, we estimate that the $33.4 \mathrm{~g}$ Ge detector in 2.4 days sees about 1.5 tracks from internal radioactivity in the sapphire and Teflon holders.

Regarding the environmental gamma-ray backgrounds inside the EDELWEISS cryostat, we may use numbers from the $860 \mathrm{~g}$ Ge EDELWEISS-III detector that operates in a similar environment [190]. For this detector, the total integrated rate from $100 \mathrm{keV}$ to $4 \mathrm{MeV}$ is about 370 counts/ $\mathrm{kg} / \mathrm{day}[189,190]$. If this same rate is incident on the sapphire and teflon, then the number of tracks due to environmental radioactivity in the 2.4 days of exposure is about 0.3 . Therefore, summing the contributions from high-energy particles from the ambient radioactive background and from impurities, we expect at most a couple of tracks passing through the Teflon and sapphire during the detector's exposure, which leads to at most two events. 
The EDELWEISS data show, however, an event rate that is several orders of magnitude higher. We conclude that the observed backgrounds cannot be due to any type of radiation coming from tracks in the main uninstrumented materials surrounding the detector.

Looking toward the future, it is worth pointing out that the radioactive high-energy tracks discussed above, even if rare, do still contribute a sizable low-energy background rate. Since both sapphire and Teflon are dielectric materials, tracks passing through them lead to Cherenkov radiation. Cherenkov radiation in sapphire is discussed in Sec. II A, where we find that tracks with energies above approximately $100 \mathrm{keV}$, which is in the typical energy range of radioactive tracks, lead to Cherenkov photons. In addition, electrons and holes produced by tracks in sapphire are shown to produce luminescence due to defects, leading to approximately $3 \mathrm{eV}$ photons [191]. Regarding Teflon, the dielectric constant of Teflon is measured in Ref. [192] to be about $\epsilon_{\mathrm{PTFE}} \sim 1.6$ in the energy range $0.8-3 \mathrm{eV}$, rising to $\epsilon_{\mathrm{PTFE}} \sim 2.1$ at an energy of $8.8 \mathrm{eV}$; this implies that Cherenkov photons can be generated from electrons with energies above approximately $200-300 \mathrm{keV}$, which again is an energy range that can be expected from radioactive events. Consequently, given the high-energy track rate discussed above, we expect $\mathcal{O}(1)$ events per day associated with Cherenkov radiation from such tracks. A detailed modeling of the Cherenkov and luminescence backgrounds is, therefore, warranted to understand more precisely the implications for the future sensitivity of this detector setup to sub-GeV dark matter.

\section{Radiative backgrounds at other existing experiments}

We briefly comment on radiative backgrounds in a few other experiments that observe low-energy events above what is expected from Compton and other radiogenic backgrounds.

(i) CRESST-III $[61,62]$.- -Using a $23.6 \mathrm{~g} \mathrm{CaWO}_{4}$ cryogenic crystal target, the CRESST-III Collaboration achieves a nuclear recoil threshold of $30.1 \mathrm{eV}$ and an exposure (after cuts) of $3.64 \mathrm{~kg}$-days. The target is well shielded and placed underground at the Laboratori Nazionali del Gran Sasso). An excess of events above that expected from radioactive backgrounds is observed at energies well below approximately $200 \mathrm{eV}$.

We find that it is unlikely for Cherenkov radiation, transition radiation, or luminescence to be an important background in the final dataset after cuts are applied, since all holders of the $\mathrm{CaWO}_{4}$ crystal target and the vast majority of nonconductive material inside the copper housing is designed to scintillate if it interacts with a high-energy particle. In this way, while many Cherenkov and possibly recombination photons are generated inside the copper housing, it is easy to veto them by observing the accompanying scintillation from the high-energy event that generates the radiation.

Research into the origin of the low-energy events is ongoing, and plausible hypotheses that are being investigated include cracking or microfracturing of the crystal or support holders that occur when the detectors are being tightly clamped [84]. Another possibility that deserves investigation is luminescence from the various detector materials that occurs only well after a track passes through it (phosphorescence).

(ii) SuperCDMS CPD [72].-Using a $10.6 \mathrm{~g} \mathrm{Si}$ athermal phonon detector with an energy resolution of approximately $5 \mathrm{eV}$ and a raw exposure of 9.9 g-days, the SuperCDMS Collaboration obtains the strongest constraints on elastic dark-matter-nucleon scattering for dark-matter particle masses from 87 to $140 \mathrm{MeV}$. The detector is operated on the surface, where it is subject to a large environmental background rate of approximately a few $\times 10^{5} \mathrm{DRU}$ at $\mathcal{O}(\mathrm{keV})$ energies. A large number of events-much larger than that expected from background photons that Compton scatter in the target $\mathrm{Si}$-are observed for recoil energies below approximately $150 \mathrm{eV}$.

Radiative backgrounds may arise, however, from uninstrumented auxiliary materials. The detector is clamped between six Cirlex holders (three on the top and three on the bottom), which can generate radiation when high-energy electrons and muons interact with them. The CPD detector operates without a bias voltage, has very little position resolution (so radiated photons that interact at the surface of the detector likely cannot be vetoed), and has only a single detector inside the copper housing (so photons cannot be vetoed by using a coincident signal between two or more detectors). We expect Cherenkov or recombination radiation to be dominantly generated by environmental background radioactive events incident on the Cirlex clamps (rather than from impurities in the Cirlex) and transition radiation to be generated from environmental backgrounds and cosmic rays passing through the various inner-detector layers. Including muons from Ref. [187] and naively scaling up the number of tracks observed in the SENSEI MINOS data [68] to the background rate observed in the CPD detector at $\mathcal{O}(\mathrm{keV})$ energies reveals that radiation from tracks passing through the Cirlex cannot make up for more than $10 \%$ of the observed signal. Given the large observed event rate in the CPD detector, this implies that radiation from tracks, which could, for instance, arise via the Cherenkov effect, still constitutes a significant background in this experiment, even if in this case it is subdominant to whatever else is causing the low-energy events. 
(iii) EDELWEISS-Surf Ge bolometer [48].-Using a $33.4 \mathrm{~g} \mathrm{Ge}$ cryogenic target operated with a neutron-transmutation-doped Ge thermal sensor, the EDELWEISS Collaboration achieves a heat-energy resolution of $17.7 \mathrm{eV}$ and constrained elastic darkmatter-nucleon scattering for dark-matter masses above approximately $600 \mathrm{MeV}$. An excess above an approximately flat Compton background is observed for recoil energies below approximately $150 \mathrm{eV}$. As discussed above in Sec. V C, Cherenkov radiation can be generated from high-energy electrons and muons interacting with the Teflon (PTFE) holders. Transition radiation can also be generated from the passage of high-energy events through various detector layers. A detailed investigation of what fraction of the observed excess events is from such radiative backgrounds is beyond the scope of this paper.

(iv) Optical haloscopes [193]._-Dark matter may be composed of light bosonic fields, which behave as a background dark-matter oscillation. Using layers of dielectric materials, Ref. [193] proposes to enhance the conversion of the background dark matter, dark photons, in particular, into visible photons. The visible photons can be focused into a small superconducting nanowire detector using a lens. [194] In this setup, backgrounds can arise from tracks that pass through the lens, which emit a large number of Cherenkov photons that mimic the darkmatter signal.

\section{RADIATIVE BACKGROUNDS AT FUTURE AND PROPOSED EXPERIMENTS}

In this section, we discuss radiative background at the upcoming SuperCDMS SNOLAB experiment [21], comment briefly on upcoming and proposed large-exposure Skipper CCD detectors (SENSEI at SNOLAB, DAMIC-M, and Oscura) [88,89], and also comment on proposed experiments based on athermal phonon detection and low-energy scintillation $[12,29,44,59,90,195]$.

\section{A. SuperCDMS at SNOLAB}

The upcoming SuperCDMS at SNOLAB experiment will use two types of cryogenic detectors, denoted HV and iZIP, and two types of target materials, namely, Si and Ge [21]. The goal is to operate the HV detectors with a bias voltage (approximately $100 \mathrm{~V}$ ) that amplifies ionization signals produced in the target material by drifting electrons and creating phonons via the Luke-Neganov-Trofimov effect $[179,180]$. We estimate below the expected background from Cherenkov photons produced from nonconductive materials inside the detector housing for the HV detectors. We argue that the Cherenkov background may dominate over other previously considered backgrounds at low energies, but a careful simulation and more details about the optical properties of Cirlex are needed to estimate precisely this background. Additional details about our estimate can be found in Appendix D. We do not attempt here an estimate of transition radiation or of the radiative recombination rate in Cirlex.

At SuperCDMS SNOLAB, there will be eight HV detectors with a Ge target, each with a mass of $1.39 \mathrm{~kg}$, and four HV detectors with a Si target, each with a mass of $0.61 \mathrm{~kg}$. These targets are placed in two separate towers, with each tower surrounded by its own copper housing and with each tower consisting of four $\mathrm{Ge}$ and two Si HV detectors. Each HV detector inside this tower is held by 12 clamps (six on the top and six on the bottom) made of Cirlex. Each clamp has a mass of $0.17 \mathrm{~g}$. In addition, four straight and four curved detector interface boards made of Cirlex sit on the edge of the copper housing (near the top and bottom of each HV detector) and have a direct view to the HV detector; the mass of each such detector interface board is approximately $0.21 \mathrm{~g}$. The total mass of Cirlex that sits inside or along the side of the copper housing for each $\mathrm{HV}$ detector is, therefore, about $3.72 \mathrm{~g}$, or about $22.3 \mathrm{~g}$ for one tower consisting of six HV detectors.

Cirlex is a nonadhesive laminate made from $100 \%$ Kapton polyimide film [196]. Cirlex has the same chemical, physical, thermal, and electrical properties as Kapton polyimide. Since we are unable to find in the literature the measured optical properties of Cirlex specifically, we assume in our estimate below that its optical properties are the same as for Kapton polyimide [197,198]. Kapton polyimide has a density of $1.43 \mathrm{~g} / \mathrm{cm}^{3}$ and a dielectric constant of $\epsilon \sim 3$ [197] [which varies $\lesssim 10 \%$ for energies in the relevant $\mathcal{O}(\mathrm{eV})$ range; see below]. Cherenkov photons can, thus, be generated from electrons that have a kinetic energy $\gtrsim 115 \mathrm{keV}$.

The dominant source of high-energy electrons comes from beta decays of the radioactive impurities ${ }^{238} \mathrm{U},{ }^{232} \mathrm{Th}$, and ${ }^{40} \mathrm{~K}$, which are present in the Cirlex [21]. These radioactive impurities are found to have the following concentrations: approximately $14 \mathrm{mBq} / \mathrm{kg}$ for ${ }^{238} \mathrm{U}$, approximately $4.5 \mathrm{mBq} / \mathrm{kg}$ for ${ }^{232} \mathrm{Th}$, and $\lesssim 5.3 \mathrm{mBq} / \mathrm{kg}$ for ${ }^{40} \mathrm{~K}[199,200]$. Based on this information, we estimate that, in the $22.3 \mathrm{~g}$ of Cirlex present in one tower of six detectors, the rate for producing energetic electron tracks from radioactivity is approximately 200 events per day or approximately 75000 events per year. A significant fraction of these tracks have energies above the $115 \mathrm{keV}$ threshold for the production of Cherenkov photons in Cirlex, namely,

$N_{\text {events }}^{\text {Cirlex }} \sim 130$ events per day $\sim 50000$ events per year.

In addition, the Cirlex may have some luminescence (from, e.g., recombination of $e^{-} h^{+}$pairs), and we are unaware of 
measurements in the literature that constrain this possibility. We focus our remaining discussion in this subsection on Cherenkov radiation, although some of our comments are also applicable to luminescence.

To understand the possible signals generated in the HV detectors by these beta decays, we need to know how many Cherenkov photons are produced and how many of these are detected in the HV detectors. Cirlex (assuming it has the same optical properties as Kapton polyimide) has a direct band gap of approximately $1.5 \mathrm{eV}$ and an indirect band gap of approximately $1.8 \mathrm{eV}$ [198], so that photons with energies $\gtrsim 1.5 \mathrm{eV}$ are likely absorbed quickly once produced. However, photons with energy $\lesssim 1.5 \mathrm{eV}$ could leave the Cirlex and be absorbed in the HV detector. As discussed in Sec. II A (cf. Fig. 5), the number of Cherenkov photons emitted by an electron depends on the track energy, which sets the track length. The largest number of Cherenkov photons that can be generated by the above radioactive contaminants comes from the highest-energy beta decay, which is $3.27 \mathrm{MeV}$ at the end point of the ${ }^{214} \mathrm{Bi}$ decay, which appears in the ${ }^{238} \mathrm{U}$ decay chain. The electron from this beta decay could travel as much as approximately $12.7 \mathrm{~mm}$ inside the Cirlex and produce a maximum of approximately 106 (approximately 236) Cherenkov photons, with energies uniformly distributed in the range 1.16$1.5 \mathrm{eV}(0.74-1.5 \mathrm{eV})$, where the lower bound on the energy is chosen to correspond to the $\mathrm{Si}(\mathrm{Ge})$ band gap [108] and the upper bound corresponds to the direct energy gap of the Cirlex. In contrast, an electron with an energy of $200 \mathrm{keV}$ on average produces only about 1.3 (about 3.0) Cherenkov photons in the relevant energy range for $\mathrm{Si}(\mathrm{Ge})$.

A careful simulation is needed to determine how many Cherenkov radiation events make it to the detector and remain after several obvious analysis cuts. First, a gamma ray or $\mathrm{x}$ ray may accompany the beta decay and be absorbed in an HV detector, allowing the event to be vetoed. Second, events that produce many Cherenkov photons can be vetoed, since they produce a coincident signal in two or more HV detectors; however, events that produce only a few photons may be difficult to remove with an anticoincidence veto. Another useful discriminant could be to veto events that do not penetrate deeply into the cylindrical side walls of the HV detectors. This allows one to veto some events that produce Cherenkov photons that all hit a single detector, since such events often contain multiple photons, some of which are well above the $\mathrm{Si}$ or Ge band gap and, hence, are absorbed on the surfaces. However, even in this case, some beta decays could produce a few photons, all of which are within approximately $0.1-0.2 \mathrm{eV}$ of the Si or Ge band gaps, so they have a large absorption length and penetrate into the bulk.

In Ref. [21], the expected number of events (before any analysis cuts) with energy $\lesssim 50(100) \mathrm{eV}$ in the HV detectors, from the HV detector-bulk contamination and ambient background incident on the HV detectors, is about
9 (18) events per year in one Si HV detector and about 2 (4) events per year in one Ge HV detector. Based on our discussion above, it is conceivable that the Cherenkov background dominates in this energy range over the backgrounds that were considered previously. This would affect the projected SuperCDMS SNOLAB sensitivity on various dark-matter models. However, a careful study of the optical properties of Cirlex and a detailed simulation of the Cherenkov-photon background is needed to determine these implications precisely and whether this background is a real concern. In addition, measurements that determine the luminescence of the Cirlex would also be important. Appendix D contains additional details relevant to our discussion above, as well as brief comments on the possibility of operating some, or all, of the six HV detectors with a zero bias voltage.

\section{B. Future Skipper CCD experiments}

We will discuss in Ref. [178] the implications of radiative backgrounds for the future Skipper CCD searches SENSEI at SNOLAB (100 g), DAMIC-M at Modane (1 kg) [88], and Oscura (10 kg) [89]. We note, however, that these upcoming and proposed experiments will have a drastically improved shielding, which will reduce radiative backgrounds coming from high-energy tracks. For example, SENSEI at SNOLAB is aiming for a background rate that is about 3 orders of magnitude better [approximately 5 DRU at $\mathcal{O}(\mathrm{keV})$ energies] than the SENSEI data taken at MINOS [approximately $3000 \mathrm{DRU}$ at $\mathcal{O}(\mathrm{keV})$ energies [68]], while DAMIC-M and Oscura are aiming for a background rate of about 0.1 and 0.01 DRU, respectively. This would lead to a very low single-electron-event background rate from trackinduced radiation, but a careful analysis of these backgrounds is needed.

\section{Future dark-matter detectors searching for photons with scintillators and molecules}

Radiative backgrounds could mimic a dark-matter signal at proposed detectors that search for one or more photons from dark-matter interactions in, e.g., solid-state scintillators [12], molecular targets [44,195], and organic aromatic materials [59]. Radiative backgrounds could either excite the target material, which subsequently produces a photon, or directly hit the photodetector.

As a concrete example, we consider the proposed SPICE detector, which aims to detect the scattering or absorption of light-dark matter on a GaAs target by measuring a scintillating signals obtained from the event $[12,90]$ (SPICE also aims to detect phonons from dark-matter events; see Sec. VID). The proposed detector setup consists of multiple targets with a volume of the order of $\mathrm{cm}^{3}$ each, with independent sensing devices attached to each target. Cherenkov or recombination radiation from high-energy tracks passing through noninstrumented dielectric materials, such as the detector holders, or transition radiation from 
high-energy tracks passing through two or more layers with different refractive indices, can produce photons that could make it into their detector target. In the GaAs target, photons with energy $\gtrsim 1.5 \mathrm{eV}$ can be directly absorbed by the photodetector or they can be absorbed by the GaAs target that subsequently scintillates and emits a secondary photon; in both cases, such photons mimic a dark-matter signal.

\section{Future dark-matter detectors searching for collective excitations}

The search for collective modes in solid-state detectors, such as phonons and magnons, has been suggested as a way to probe sub-MeV dark-matter scattering off, and sub-eV dark matter being absorbed by, a target material $[29,54,56]$. The background processes discussed here can also mimic dark-matter signals for these searches.

As a concrete example, we again consider the SPICE detector (see also Sec. VI C). One of the goals with SPICE is to detect the scattering or absorption of matter on a sapphire target by measuring sub-eV phonon signals obtained from the event [90]. While achieving sub-eV energy resolutions is currently still challenging, the proposal aims to develop novel transition-edge sensors to obtain the required sensitivities.

The authors of the proposal identify two possible backgrounds affecting future dark-matter searches in their detectors, neutrinos and radiogenic backgrounds, and indicate that, after discriminating high-energy events from their low-energy signals, zero backgrounds are to be expected [29]. Another potential background comes from the low-energy coherent scattering of a photon off nuclei [201], but these can likely be mitigated using an active veto. However, backgrounds such as Cherenkov, transition, or recombination radiation, as well as nonradiative recombination, have not been discussed previously in the literature.

Regarding Cherenkov or recombination radiation, highenergy tracks passing through noninstrumented dielectric materials, such as the detector holders, lead to photons with a variety of energies. Such photons may escape the noninstrumented materials and make it into their detector target. In particular, Cherenkov photons can have energies that match the lattice modes of the sapphire detector. In this case, they convert into optical phonons in the target and, thus, constitute a background. A plausibility argument for this background can be made as follows. Take the radioactivity of the Teflon holders at EDELWEISS as an example $(10 \mathrm{mBq} / \mathrm{kg})$, a total mass of $1 \mathrm{~g}$ in holders made of some insulating material, a detector with $1 \mathrm{~kg}$ of fiducial target, and an effective exposure of $10 \mathrm{~kg}$-year as indicated in Ref. [90]. In this case, $\mathcal{O}(3000)$ charged tracks are expected in the holders. According to the estimate in Eq. (2.10), each track leads to $\mathcal{O}(1)$ Cherenkov photons with energies below $100 \mathrm{meV}$, which may then convert to phonons in the sapphire detector. It is likely that most of these events are vetoed, as tracks also radiate higher-energy Cherenkov photons, which lead to large energy depositions in the detector that are not consistent with the dark-matter signal region. However, there also are low-energy charged tracks; in this case, Cherenkov photons are radiated only when the dielectric constant of the insulating holders is extremely large, which happens only near lattice modes of the holders, as discussed in Sec. II A. Thus, such tracks emit only Cherenkov photons with energies of the order of phonon modes (approximately $100 \mathrm{meV}$ and below). These events, although rare, are likely not vetoed and could pass as a dark-matter signal. A concrete example of this possibility is shown in Fig. 5, where we see that a $20-\mathrm{keV}$ electron track in $\alpha$-quartz leads to Cherenkov radiation only near lattice modes. It may be desirable, therefore, for any material near the detector to consist of a nonpolar material, of a polar material with lattice modes that do not match the lattice modes of the target, or of a material that does not produce Cherenkov radiation altogether like a conductor. However, even in this case photons may be generated by transition radiation, which needs to be studied carefully.

Another potential background for a phonon search is nonradiative recombination. A track passing through uninstrumented materials leads to a large number of $e^{-} h^{+}$ pairs. As discussed in Sec. IV, these pairs may relax and recombine emitting phonons or go toward band minima via the emission of phonons. If an uninstrumented material is in contact with the detector, a few such phonons may be transferred into the detector, mimicking the dark-matter signal.

In all cases, a precise characterization of the properties of all material surrounding the detector is crucial to avoid these low-energy backgrounds.

\section{RADIATIVE BACKGROUNDS AT NEUTRINO EXPERIMENTS AND SUPERCONDUCTING QUBITS}

Up to this point, we focus on sub-GeV dark-matter searches, but it is worth noting that the backgrounds that we identify could also be a concern for other research areas. For example, these backgrounds also appear in searches for coherent scattering between neutrinos and nuclei with Skipper CCDs such as CONNIE [95] and $\nu$ IOLETA [96].

Moreover, the radiative backgrounds we point out in this paper could have a significant impact on quantum computing, as they could limit the coherence time of superconducting qubits. It has been shown in several references [97-100] that high rates of charged particles from radioactivity and cosmic rays correlate with high quasiparticle (broken Cooper pairs) densities in superconducting qubits and reduced coherence times. The current explanation is that phonons generated by these charged tracks in a dielectric substrate interact with the qubits and create quasiparticles. However, the impact on qubit coherence times of low-energy photons from 
Cherenkov radiation, transition radiation, and luminescence from recombination is not discussed in the literature. As mentioned in previous sections, the Cherenkov process and luminescence from recombination in semiconductors typically lead to photons with energies $\mathcal{O}(\mathrm{eV})$ or below. Superconducting qubits made of $\mathrm{Al}$ efficiently absorb photons at these energies. Once absorbed, these low-energy photons can break Cooper pairs and create quasiparticles. Therefore, low-energy photons have similar effects on qubits as phonons, and they must be carefully investigated. While a detailed analysis is beyond the scope of this paper, we now present a simple estimate that demonstrates the relevance of Cherenkov and recombination photons produced by tracks passing through the dielectric substrate.

We focus on photons near or below the band gap, since, after being produced, they can travel sufficiently far in the substrate to eventually reach the qubits and create quasiparticles. Let us first consider Cherenkov photons. A single track passing through the substrate leads to approximately $\alpha \times E_{g} \times \Delta x$ subgap Cherenkov photons, where $\Delta x$ is the track length [see Eq. (2.4)]. Thus, the total energy in subgap Cherenkov photons created by a single track is approximately $\alpha \times E_{g}^{2} \times \Delta x$. For example, a sapphire $\left(\mathrm{Al}_{2} \mathrm{O}_{3}\right)$ substrate has band gap of approximately $7 \mathrm{eV}$. Thus, a $300-\mu \mathrm{m}$ track passing through such substrate can release approximately $200 \mathrm{eV}$ in the form of long-lived, subgap Cherenkov photons, which can create a large number of quasiparticles as they are absorbed by the qubits. Note that a substrate with a larger band gap produces more energy that can be absorbed by the qubit.

Moreover, substrates with high radiative recombination efficiency produce a large number of luminescence photons as tracks pass through. For instance, the luminescent yield of sapphire is approximately $1 \%$ [202], which means that an approximately $100-\mathrm{keV}$ track can release more than $1 \mathrm{keV}$ in the form of $\mathcal{O}(\mathrm{eV})$ photons. Since the sapphire substrate is basically transparent to these low-energy photons, almost all such photons are absorbed in the qubits if there are no other absorptive materials.

These consideration suggest that materials with low band gaps and low radiative recombination efficiency, such as $\mathrm{Si}$ and $\mathrm{Ge}$, could dramatically reduce radiative backgrounds for superconducting qubits and may, thus, constitute optimal substrates to mitigate them.

\section{CHARACTERIZATION AND MITIGATION OF RADIATIVE LOW-ENERGY BACKGROUNDS}

The radiative backgrounds discussed in this paper have significant implications for future searches of low-mass dark matter, both in designing future detectors and in calculating their low-energy backgrounds. Moreover, these backgrounds affect searches for dark-matter-induced electromagnetic excitations (electrons or photons) and excitations of collective modes (such as one or more phonons or magnons). Fortunately, there are several mitigation and characterization strategies. Several of these strategies are already employed to reduce radiogenic backgrounds and are not specific to mitigating the backgrounds discussed in this paper, but others are not universally used.

(i) Increase passive shielding.-Passive shielding with lead, copper, and other materials reduces the number of photons that can interact in the detector or the material that surrounds it, thereby reducing the number of backgrounds from Cherenkov radiation, transition radiation, recombination, and bremsstrahlung.

(ii) Increase active shielding.-In detectors with excellent timing resolution, an active veto can detect a high-energy event in coincidence with a low-energy event, in which case the low-energy event can be vetoed. For example, the CRESST-III detector partially employs an active shield, for which the materials inside the copper housing scintillate when interacting with a high-energy event.

(iii) Minimize nonconductive materials near sensors.Reducing nonconductive materials and, when feasible, either replacing them with conducting materials or covering them with a conductive surface reduces Cherenkov radiation and recombinationinduced backgrounds. It may also be possible to add coatings on the nonconductive materials to absorb low-energy photons, although it is important to check that the coating itself does not lead to additional radiation that can mimic low-energy events in the sensor. We note that transition radiation can be generated in all materials.

(iv) Radiopure materials. - In addition to high-energy events from ambient background radiation, a second, often dominant, source of high-energy events can arise from radioactive contaminants inside the detector materials. These can, for example, beta decay to produce electrons that create Cherenkov radiation. The careful selection and screening of materials are crucial to reduce these sources of highenergy events that can create Cherenkov radiation, transition radiation, and recombination-induced backgrounds.

(v) Multiple sensors.-Having multiple nearby sensors (inside a common copper shield) can drastically reduce low-energy events that mimic dark matter by allowing events to be vetoed in which two or more sensors detect events in the same time window. For example, Cherenkov radiation often produces multiple low-energy photons, which can be vetoed if the photons get absorbed in two or more sensors. However, Cherenkov radiation in which only one or a few photons are produced that are all absorbed by one sensor still constitutes a background. It may also be challenging to veto transition radiation using multiple sensors, since few photons are typically produced. 
(vi) Precise measurements of properties of all materials.-Our work makes it clear that the low-energy backgrounds at different experiments depend very sensitively on the detailed properties of detector materials. Estimating the low-energy background rates requires precise knowledge of each material's relative permittivity and extinction coefficient, recombination coefficients, electron and hole mobilities, etc., all of which must be specified at the detector's operating temperature. These properties are often not known precisely in the literature for the materials used in different dark-matter detectors and so should be measured in each case.

(vii) Thinning of back-side Skipper CCDs.-The back side of CCDs is routinely thinned for astronomical applications, but it naively seems not to be necessary for dark-matter searches. However, we show that a careful study of the back-side thickness and doping levels is needed. A "thick" layer heavily doped with phosphorus helps absorb near-band-gap photons from Cherenkov and radiative $e^{-} h^{+}$recombination, preventing such photons from traveling far away from the high-energy charged particle track. However, such a thick layer also leads to many photons from radiative $e^{-} h^{+}$recombination. We will discuss this further in Ref. [178].

(viii) Thin layer of absorptive material on surfaces.-It may be useful to cover all surfaces with a thin layer of material that absorbs low-energy photons. Such layers may create more radiative backgrounds but could be beneficial if they absorb light that can create events in the target.

(ix) Substrate with low band gap and low luminescence for superconducting qubits.-As mentioned in Sec. VII, low-energy photons generated from the substrate can create quasiparticles in qubits and reduce the coherence time. Using substrates with low band gaps and small luminescence rates, such as $\mathrm{Si}$ or Ge, could alleviate these backgrounds.

The mitigation strategies above quite plausibly reduce these backgrounds to manageable levels. However, a detailed investigation of these backgrounds is necessary for each experiment.

\section{SUMMARY AND CONCLUSIONS}

In this paper, we discussed four processes that can produce low-energy events in low-threshold direct-detection experiments and which have not been previously considered as important backgrounds: Cherenkov radiation, transition radiation, and luminescence or phonons from recombination. These processes can generate lowenergy (approximately $\mathrm{meV}$ to few-eV) photons or phonons that can be absorbed by the target or sensor and, depending on the specific target properties, create one or a few $e^{-} h^{+}$pairs, phonons, or photons that can mimic the signal expected from a wide range of sub-GeV dark-matter interactions. Cherenkov radiation is generated when highenergy charged particles (electrons and muons, from cosmic rays or radioactivity) above some threshold energy interact with the detector target material or any nonconductive material surrounding the target. Transition radiation is generated when charged particles cross the boundary between two materials with different relative permittivities. Recombination can occur between electron and holes generated after the passage of a high-energy charged track.

We discussed these backgrounds in the context of several existing and proposed experiments.

(i) A sizable fraction of the approximately 450/g-day of single-electron events seen in SENSEI data from a Skipper CCD at a shallow underground site arise from Cherenkov radiation and radiative recombination. Cherenkov photons close to the Si band gap are generated by charged particles interacting (mostly) in the Si Skipper CCD, which can travel "far" away from the high-energy track before being absorbed. In addition, photons close to the band gap are also generated by the radiative recombination of $e^{-} h^{+}$ pairs created by charged tracks in an $\mathcal{O}(\mu \mathrm{m})$ layer of Si heavily doped with phosphorus on the back side of the Skipper CCD. Transition radiation provides a negligible contribution to the observed rate. We defer to a companion paper [178] a precise calculation of these backgrounds, which is challenging and must include a careful modeling of the Skipper CCD structure near and on its surfaces. The above backgrounds needs to be evaluated carefully for future searches using Skipper CCDs, including SENSEI at SNOLAB, DAMIC-M, and Oscura, although all of them will be greatly aided by the planned excellent passive shielding.

(ii) We find that Cherenkov and transition radiation provide a plausible origin of the events containing 2-6 electrons in the SuperCDMS HVeV surface data. Cherenkov radiation, which likely dominates over transition radiation, is produced in the printedcircuit boards and plastic connectors located inside the copper housing near the $\mathrm{Si} \mathrm{HVeV}$ detector, while transition radiation is produced when charged tracks cross into the copper housing or between two materials. A detailed simulation and the detailed optical properties (currently unavailable) of the nonconductive materials in the detector housing are required to evaluate these backgrounds precisely.

(iii) Even experiments that are well shielded could be affected by these backgrounds. For example, we showed that Cherenkov radiation from radioactive contaminants in the approximately $0.35 \mathrm{~g}$ of Teflon and sapphire holders may produce a raw rate of $\mathcal{O}(1)$ potential low-energy events per day in the EDELWEISS Ge detector. This is too small to explain their currently observed low-energy event rate but is 
sufficiently large to warrant a careful investigation for future searches.

(iv) We find that our backgrounds are unlikely to explain the excess events observed at CRESST-III [61,62], since the materials inside the copper vessel scintillate when interacting with a high-energy particle and Cherenkov events would, thus, be vetoed.

(v) We find that Cherenkov radiation or luminescence would not be able to explain more than about $10 \%$ of the large observed low-energy event rate at the SuperCDMS CPD detector [72].

(vi) We pointed out that beta decays of radioactive contaminants $\left({ }^{238} \mathrm{U},{ }^{232} \mathrm{Th}\right.$, and $\left.{ }^{40} \mathrm{~K}\right)$ in the approximately $22.3 \mathrm{~g}$ of Cirlex located inside or on the copper side walls of a tower of six SuperCDMS SNOLAB detectors will produce a raw rate of approximately 75000 potential events per year containing one to several Cherenkov photons. While not all will make it to the detector and while most can be easily vetoed, a careful analysis is needed to determine the surviving background.

(vii) We discussed how the radiative backgrounds could also mimic a dark-matter signal in proposed searches in which the signal consists of one or more photons. Typical targets consist of, e.g., solid-state scintillators (as for the SPICE detector), molecular gases, or organic aromatic materials.

(viii) We also showed that the radiative and nonradiative backgrounds can mimic a dark-matter signal in proposed searches in which the signal consists of one or more phonons (such as SPICE). In particular, subgap photons with energy near the phonon modes of the target material can be produced in materials surrounding the target and be absorbed by the target to produce a phonon. Phonons from $e^{-} h^{+}$recombination generated in detector clamps or holders may also constitute backgrounds.

(ix) Finally, we pointed out that the backgrounds that we have identified could also be a concern for other, non-dark-matter experiments. Examples include searches for coherent scattering between neutrinos and nuclei with Skipper CCDs (such as CONNIE) and the decoherence of superconducting qubits.

Fortunately, having now identified these unexplored radiative and nonradiative backgrounds, we can point out several mitigation and characterization strategies. Mitigation strategies include using a large passive shield, using an active shield, using radiopure materials, minimizing nonconductive materials near the target material and the sensors, using multiple nearby sensors to veto coincident low-energy events, and (for detectors using Skipper CCDs) investigating if thinning the back side of CCD detectors can reduce backgrounds. The first three strategies are already employed to reduce radiogenic backgrounds, but the latter are not universally used.
In addition, a careful calculation of the backgrounds studied here is of utmost importance, as it could enable the different experimental collaborations to perform background subtraction and enhance their reach to detect dark matter. Calculating these backgrounds requires detailed and detector-specific simulations, which could be carried out with GEANT4 [203], using the input from theorists, the different experimental collaborations, and GEANT4 developers. Note that these simulations also require precise knowledge of the optical properties of all materials in the detector. While these are not necessarily difficult to measure, they are not currently available to sufficient precision for many materials commonly found in darkmatter detectors.

Finally, we note that there are other radiative backgrounds that we did not consider in detail, such as bremsstrahlung and diffraction radiation. The bremsstrahlung rate is $\mathcal{O}\left(\alpha^{3}\right)$ but enhanced at low photon-emission energies and at low charged-particle energies. However, a simple estimate suggests, for example, that its contribution to the single-electron-event rate in the SENSEI MINOS data is about 2 orders of magnitude less than the observed rate. Diffraction radiation occurs when a charged particle travels close to the interface between two materials. We leave a detailed study of these and other backgrounds to future work.

\section{ACKNOWLEDGMENTS}

We are especially grateful to Donald Groom, Stephen Holland, Noah Kurinsky, Ben Loer, Javier Tiffenberg, and Sho Uemura for several extensive discussions and correspondence. We have also benefitted greatly from numerous insightful discussions and/or correspondence with Quentin Arnaud, Julien Billard, Mariano Cababie, Alvaro Chavarria, Cyrus Dreyer, Juan Estrada, Marivi Fernández-Serra, Enectali Figueroa-Feliciano, Yonatan Ben Gal, Jules Gascon, Daniel Gift, Alexandre Juillard, Ben Loer, Guillermo Fernandez Moroni, Sravan Munagavalasa, Federica Petricca, Franz Pröbst, Matt Pyle, Florian Reindl, Alan Robinson, Jochen Schieck, Aman Singal, Christian Strandhagen, Raimund Strauss, Sho Uemura, Jerry Va'vra, and Tomer Volansky. R. E. acknowledges support from Department of Energy Grant No. DE-SC0009854, Simons Investigator in Physics Grant No. 623940, the Heising-Simons Foundation Grant No. 79921, and the U.S.-Israel Binational Science Foundation Grant No. 2016153. P.D. and M. S. are supported in part by Simons Investigator in Physics Grant No. 623940. P. D. is also supported in part by National Science Foundation Grant No. PHY-1915093. D. E.-U. is supported by Perimeter Institute for Theoretical Physics. Research at Perimeter Institute is supported in part by the Government of Canada through the Department of Innovation, Science and Economic Development Canada 
and by the Province of Ontario through the Ministry of Economic Development, Job Creation and Trade.

\section{APPENDIX A: CARRIER CONCENTRATIONS IN SEMICONDUCTORS}

The background (equilibrium) number densities of electron and holes in a semiconductor, $\bar{n}_{e, h}$, are determined by the intrinsic concentration and the density of dopants. The intrinsic carrier concentration corresponds to the density of electrons and holes in the absence of dopants, purely due to the thermal excitation of electrons into the conduction band. Approximating the band structure as parabolic, and, assuming a nondegenerate semiconductor, the intrinsic carrier concentration is given by [147]

$$
\begin{aligned}
& n_{i}=\sqrt{N_{C} N_{V}} e^{-E_{g} / 2 T}, \\
& \bar{n}_{e}=\bar{n}_{h}=n_{i} \quad \text { (intrinsic semiconductor), }
\end{aligned}
$$

where $E_{g}$ is the band gap and $N_{C}\left(N_{V}\right)$ is the effective density of states in the conduction (valence) band. The effective densities of state are [147]

$$
\begin{aligned}
& N_{C}=2\left[\frac{m_{e}^{*} T}{2 \pi}\right]^{3 / 2}, \\
& N_{V}=2\left[\frac{m_{h}^{*} T}{2 \pi}\right]^{3 / 2},
\end{aligned}
$$

where $m_{e, h}^{*}$ are the electron and hole effective masses. We present the band gaps and effective masses in Table III for $\mathrm{Si}, \mathrm{Ge}$, and GaAs.

When considering doped materials, the background value of the electron and hole densities $\bar{n}_{e, h}$ differs from the intrinsic carrier concentration of Eq. (A2). In this case, the number density of $n$-type ( $p$-type) dopants $n_{d}$ sets the concentration of electrons (holes). The concentration of the opposite carrier can then be obtained by using the equilibrium condition $\bar{n}_{e} \bar{n}_{h}=n_{i}^{2}$, which holds true in the presence of doping [147]. Thus, for doped materials, the carrier concentrations are

$$
\begin{array}{lrl}
\bar{n}_{e}=n_{d}, & \bar{n}_{h}=n_{i}^{2} / n_{d} & \text { (n-doped }), \\
\bar{n}_{e}=n_{i}^{2} / n_{d}, & \bar{n}_{h}=n_{d} & (\text { p-doped }) .
\end{array}
$$

The carrier concentrations in doped materials usually significantly exceed the room-temperature intrinsic carrier concentrations.

TABLE III. The room-temperature band gap, effective electron mass, and effective hole mass for $\mathrm{Si}, \mathrm{Ge}$, and GaAs.

\begin{tabular}{lccc}
\hline \hline & $\mathrm{Si}$ & $\mathrm{Ge}$ & $\mathrm{GaAs}$ \\
\hline$E_{g}(\mathrm{eV})[108]$ & 1.11 & 0.66 & 1.43 \\
$m_{e}^{*}[147]$ & $0.26 m_{e}$ & $0.12 m_{e}$ & $0.068 m_{e}$ \\
$m_{h}^{*}[147]$ & $0.39 m_{e}$ & $0.3 m_{e}$ & $0.5 m_{e}$ \\
\hline \hline
\end{tabular}

The background equilibrium density of electrons and holes determines the Fermi level of a material. For nondegenerate semiconductors and using the parabolic bandstructure approximation, the Fermi level can be obtained by using Eqs. (A2) or (A3) and the expressions

$$
\begin{aligned}
& \bar{n}_{e}=N_{C} \exp \left[-\left(E_{C}-E_{F}\right) / T\right], \\
& \bar{n}_{h}=N_{V} \exp \left[-\left(E_{F}-E_{V}\right) / T\right] .
\end{aligned}
$$

Here, $E_{C}\left(E_{V}\right)$ is the energy of the conduction (valence) band, and $E_{F}$ is the Fermi energy. For instance, in the case of an intrinsic semiconductor, using the intrinsic electron or hole density [Eqs. (A2) and (A4), respectively], we obtain

$$
E_{F}^{i}=\frac{1}{2}\left(E_{C}+E_{V}\right)+\frac{1}{2} T \log \left(N_{V} / N_{C}\right) .
$$

Note that, in an intrinsic semiconductor, the Fermi level lies approximately midways in between the valence and conduction bands. In doped semiconductors, the Fermi level can be easily found by using Eq. (A3) in Eq. (A4), with the result that in $n$-doped ( $p$-doped) semiconductors the Fermi level lies close to the conduction (valence) band.

\section{APPENDIX B: DETAILS ON CHERENKOV, TRANSITION RADIATION, AND RECOMBINATION IN SENSEI}

In this Appendix, we provide a few additional details for how we estimate Cherenkov radiation, recombination, and transition radiation rates in Sec. VA. A detailed simulation will be presented in Ref. [178].

\section{Cherenkov radiation}

For Cherenkov radiation, we consider here only those tracks that pass through the CCD and neglect radiation generated in the pitch adapter and epoxy. The charged tracks passing through the detector can be either electrons or muons, and SENSEI presents the aggregated data in Ref. [68]. Comparing with the muon-only tracks in Ref. [187], we find that muons contribute less than $10 \%$ of the number of electron tracks, so in what follows we neglect them and assume that all track data come from electrons.

Assuming that the electron events are isotropic, most electrons traverse the Skipper CCD along its shortest dimension, $675 \mu \mathrm{m}$. However, electrons can be stopped in the Skipper CCD before exiting, depending on their mean range $\ell_{e}$ shown in Fig. 6, which depends on the track energy. Thus, the typical length of an electron track is

$$
L=\min \left(675 \mu \mathrm{m}, \ell_{e}\right) .
$$

Once the photons are emitted by the charged tracks, the distance they travel away from the originating track is 
determined by the photon absorption length, which depends on the photon energy. In particular, and as discussed in Sec. II A, photons with energies close to the Si band gap may travel for macroscopic distances before converting into $e^{-} h^{+}$pairs. From Fig. 15, we see that photons with energies in the range $1.1 \mathrm{eV} \lesssim \omega \lesssim$ $1.2 \mathrm{eV}$ are absorbed 60 pixels or more away from the track. Using our electron track distribution data, the typical track length (B1), and the Si dielectric function in Fig. 3 and integrating the Cherenkov differential rate Eq. (2.4) over photon energies in the range $1.1 \mathrm{eV} \leq \omega \leq 1.2 \mathrm{eV}$, we obtain our estimate for the number of single-electron events expected at SENSEI from Cherenkov backgrounds:

$$
R_{1 e^{-}}^{\text {Cherenkov }} \sim 500 / \text { g-day. }
$$

\section{Radiative recombination}

For radiative recombination, we obtain the number and spectrum of the tracks as done for the Cherenkov estimate above. The phosphorus doping profile in the back side of the CCD is taken from Ref. [204]. The doping concentration varies from $10^{20} \mathrm{~cm}^{-3}$ close to the back side of the CCD to $10^{17} \mathrm{~cm}^{-3}$ at a distance of approximately $4 \mu \mathrm{m}$ from the back side. For each track, we obtain the number of $e^{-} h^{+}$pairs created per unit-track length in the doped region, $d N_{h} / d x$, using Eq. (4.1) and the $\mathrm{Si}$ ionization energy from Ref. [143]. A fraction of these holes recombine radiatively with the electrons donated by the dopant. This fraction is given by (see Sec. IV B)

$$
\frac{d N_{\gamma}}{d x}=\frac{d N_{h}}{d x} \frac{\tau}{\tau^{\text {direct }}},
$$

where $\tau$ is the hole lifetime and $\tau^{\text {direct }}$ the radiative recombination timescale in Eq. (4.10). Both these lifetimes depend on the electron-carrier concentration, which is set by the doping density discussed above. The radiative recombination coefficient at the SENSEI operating temperature, required to calculate $\tau^{\text {direct }}$, is obtained from Ref. [148]. Regarding the hole lifetime, we take it be the minimum between three timescales: (i) the trap-assisted recombination time, which we take to be $10^{-6} \mathrm{~s}$, (ii) the Auger recombination time, calculated in Eq. (4.22), and (iii) the diffusion time required for the $e^{-} h^{+}$pairs to diffuse away from the doped regions:

$$
\tau=\operatorname{Min}\left(10^{-6} \mathrm{~s}, \tau^{\text {Auger }}, \tau^{\text {diff }}\right) .
$$

We approximate the diffusion time by $\tau^{\text {diff }} \sim r^{2} / D_{h}$ as in Eq. (4.22), where $D_{h}$ is the hole diffusion constant, which we take from Ref. [159], and $r$ is the length over which the holes need to diffuse. We take $r=2 \mu \mathrm{m}$, which is the scale that roughly characterizes layers with the same orderof-magnitude doping, according to Ref. [204]. Then, we integrate $d N_{\gamma} / d x$ along the length of the track passing through the doped region to obtain the number of photons per track assuming that the track goes perpendicular to the CCD. We finally sum over tracks to obtain the total amount of radiation.

To obtain the radiative recombination spectrum, we use the radiative absorption coefficient from Ref. [173] in Eq. (4.9). Doping affects the Si band gap, which, in turn, affects the absorption coefficient formulas in Ref. [173], and we include this effect by correspondingly shifting the band gap using Ref. [162].

Given the total number of recombination photons and their spectrum, we obtain the fraction of them that escape the doped region into the active CCD area by using the photon absorption coefficient above, but now including also absorption by free carriers in doped $\mathrm{Si}$, described in Refs. [205,206]. As for the Cherenkov photons, we consider only the recombination photons in the energy range $1.1 \mathrm{eV} \leq \omega \leq 1.2 \mathrm{eV}$, which pass the halo-mask cut. In this way, the corresponding rate of recombination photons leading to events at SENSEI is estimated to be

$$
R_{1 e^{-}}^{\text {recombination }} \sim 500 / \text { g-day. }
$$

\section{Transition radiation}

We now estimate the contribution to single-electron events at SENSEI from transition radiation generated from charged particles passing through material surfaces. We consider only the inner walls of the copper housing, since these constitute the largest surface area compared to the other surfaces in the detector [207].

In order to be registered as an event, the photons from transition radiation need to penetrate into the CCD bulk. Most of the CCD front side is covered by the pitch adapter, and even the part that is not covered has a $0.6-\mu \mathrm{m}$ layer of polysilicon. Photons entering the back side need to penetrate at least approximately $6 \mu \mathrm{m}$ to be registered as an event. These considerations immediately limit the energies of the photons that can penetrate into the bulk. Since only photons with energy $\lesssim 1.8 \mathrm{eV}$ can travel more than approximately $6 \mu \mathrm{m}$ in silicon, we consider only photons with energies between 1.1 and $1.8 \mathrm{eV}$. We take the dielectric functions for copper from Ref. [208] at $78 \mathrm{~K}$. Since charged particles can pass the surfaces traveling in either direction, we consider both forward and backward emission at the copper-vacuum interface.

The next ingredient is the spectrum of charged particles that pass through the copper-vacuum surface. We use the cosmic muon energy and angular spectra from Ref. [187]. For determining the electron spectrum, we make some simple assumptions rather than doing a full background simulation. The measured spectrum at the Skipper CCD consists of electrons generated inside the CCD (e.g., from $\gamma$ rays) and, to a lesser extent, of electrons coming directly 
from the outside. We then assume the ambient photon spectrum inside the copper housing is the same as the measured electron spectrum, while the electrons relevant for transition radiation are generated only from photons interacting with the copper. We also assume the angular distributions are uniform. Under this assumption, the highenergy photon flux at the inner surface of the copper housing is a factor of $A_{\text {housing }} / A_{\mathrm{CCD}}$ larger than the flux at the CCD, where $A_{\text {housing/CCD }}$ is the area of inner surface of the copper housing/CCD. To estimate the flux of electrons generated inside the copper that can pass through the copper-vacuum interface, we use the (energy-dependent) mean range of electrons inside copper, $\ell_{e}^{\mathrm{Cu}}$. Assuming each photon converts all its energy to one primary electron once it is absorbed, the number of electrons escaping from the copper housing into the vacuum toward the detector is determined by the number of photons being absorbed within $\ell_{e}^{\mathrm{Cu}}$ from the copper-vacuum interface. Since the photon flux drops over a distance $L$ inside copper as $\propto e^{-L / \ell_{\gamma}^{\mathrm{Cu}}}$, where $\ell_{\gamma}^{\mathrm{Cu}}$ is the photon absorption length inside copper, the flux of electrons passing the surface can be estimated as $R_{e}^{\text {housing }}(E) \sim R_{\gamma}^{\mathrm{CCD}}\left(e^{\ell_{e}^{\mathrm{Cu}}} / \ell_{\gamma}^{\mathrm{Cu}}-1\right) A_{\text {housing }} / A_{\mathrm{CCD}}$, where $R_{\gamma}^{\mathrm{CCD}}$ is the high-energy photon flux at the CCD.

With the above simple assumptions, the rate of singleelectron events in SENSEI from transition radiation can be expressed as

$R_{1 e^{-}}^{\mathrm{TR}} \sim \sum_{i=e, \mu} \int d E R_{i}^{\mathrm{housing}}(E) \int_{1.1 \mathrm{eV}}^{1.8 \mathrm{eV}} d \omega \frac{d N_{\gamma}^{\mathrm{TR}}(E)}{d \omega}$,

where $R_{e, \mu}^{\text {housing }}(E)$ is the spectrum of electrons (muons) passing through the inner surface of the copper housing and $d N_{\gamma}^{\mathrm{TR}} / d \omega$ denotes the rate of transition radiation [see Eq. (3.1)], which includes both forward and backward contributions. We estimate

$$
R_{1 e^{-}}^{\mathrm{TR}} \sim 0.2 / \text { g-day. }
$$

These estimates for transition radiation are clearly negligible compared to those from Cherenkov [Eq. (5.1)] and recombination [Eq. (5.2)]. We do not expect our conclusions to change qualitatively once we include the other interfaces mentioned above.

\section{APPENDIX C: DETAILS ON CHERENKOV AND TRANSITION RADIATION IN SUPERCDMS HVEV}

In this Appendix, we provide a few additional details for how we estimate Cherenkov and transition radiation for SuperCDMS-HVeV [71] in Sec. V B.

\section{Cherenkov radiation}

The $\mathrm{HVeV}$ detector target is a $0.93 \mathrm{~g} \mathrm{Si}$ crystal with dimensions $1 \mathrm{~cm} \times 1 \mathrm{~cm} \times 0.4 \mathrm{~cm}$. It likely has some layers of composite elements (such as $\mathrm{SiO}_{2}$ ) on its surface, but the precise composition of the surfaces is unknown. The detector is not perfectly black and, thus, at least partially reflects some optical photons. Several nonconductive materials are located inside the copper enclosure that constitutes the SuperCDMS HVeV detector module (see Fig. 17). This includes the two PCB boards, the plastic connectors, and the flex cable. In our estimate, we include only the PCB boards, which we expect to dominate.

We now estimate the rate of Cherenkov events produced in the two PCBs and the resulting rate and spectrum of $e^{-} h^{+}$pairs observed with the $\mathrm{HVeV}$ detector. We make several simplifying assumptions. First, we need to know the high-energy background rate and spectrum. The backgrounds consist of cosmic muons and electrons from radioactivity. The muon flux is well measured, and, for our estimate, we take a muon flux at sea level of approximately $9 \times 10^{-3} /\left(\mathrm{cm}^{2} \mathrm{~s} \mathrm{sr}\right)$, with a zenith angular distribution of $\cos ^{2} \theta$ [187]. As discussed in the main text, for the high-energy electron background, we simply assume it is the same as that measured by SENSEI [68] but scaled higher by a factor of 60 .

Second, to estimate the Cherenkov radiation generated inside the PCBs, we need to know the PCB's dielectric properties and the typical track length of high-energy electrons or muons in the PCB. We do not know the precise composition of the $\mathrm{PCB}$ used in the $\mathrm{HVeV}$ detector, nor do we find in the literature a measurement of its refractive index and extinction coefficient at optical and infrared frequencies. Given this limitation, in order to perform our estimate, we simply assume that the composition of the PCB is equal to the one of the "NEMA FR4 plate" found in Ref. [185]. This assumes that the PCB is made of $61 \% \mathrm{SiO}_{2}, 15 \%$ epoxy, and $24 \%$ of bromine and oxygen. The dielectric properties of $\mathrm{SiO}_{2}$ are discussed in Sec. II A, while the epoxy properties are taken from Ref. [186]. Since Ref. [186] provides data only for epoxy between 1.8 and $6.5 \mathrm{eV}$, we simply assume the absorption length in epoxy below $1.8 \mathrm{eV}$ (above $6.5 \mathrm{eV}$ ) is constant and the same as that at $1.8 \mathrm{eV}(6.5 \mathrm{eV})$. We consider only photons with energies above approximately $1 \mathrm{eV}$, as these are energetic enough to create an $e^{-} h^{+}$event in the SuperCDMS $\mathrm{Si}$ detector. For photon frequencies $1 \mathrm{eV} \leq \omega \leq 8 \mathrm{eV}$, both $\mathrm{SiO}_{2}$ and the epoxy have a refractive index of approximately 1.5 , so we assume that the refractive index of the PCB board is $n_{\mathrm{PCB}}=1.5$, ignoring the contributions from the bromine and oxygen. We account for absorption only in the $\mathrm{SiO}_{2}$ and epoxy and neglect possible absorption due to the oxygen and bromine. With our assumptions, the threshold energy for electrons (muons) to produce Cherenkov radiation in the PCB is $E_{e}^{\mathrm{th}}=175 \mathrm{keV}\left(E_{\mu}^{\mathrm{th}}=36 \mathrm{MeV}\right)$.

The typical track length, on the other hand, is obtained from the track mean range and the PCB dimensions. For each track, we assume that its length is given by the minimum of the electron or muon mean range, $\ell_{e, \mu}(E)$ 
(where $E$ is the kinetic energy of the incoming particle), and the size of the PCB. Since the thickness of the upper PCB and lower PCB is 0.7 and $1.5 \mathrm{~mm}$, respectively, and the electrons and muons typically pass the PCB at an angle, we take the maximum track length to be $1 \mathrm{~mm}(2 \mathrm{~mm})$ for the upper (lower) PCB. The track length is, therefore, given by

$$
\begin{aligned}
& \Delta x_{\mathrm{PCB}, \text { upper }}(E)=\operatorname{Min}\left[1 \mathrm{~mm}, \ell_{e, \mu}(E)\right], \\
& \Delta x_{\mathrm{PCB}, \text { lower }}(E)=\operatorname{Min}\left[2 \mathrm{~mm}, \ell_{e, \mu}(E)\right] .
\end{aligned}
$$

For electrons, the mean range can be obtained according given the composition of FR4 from Ref. [122]. For muons, the mean range $\ell_{\mu}$ inside the PCB is much longer than the PCB sizes, so the muon path is limited to $1 \mathrm{~mm}(2 \mathrm{~mm})$ for the upper (lower) PCB.

We estimate then the total number of Cherenkov photons per high-energy charged particle as

$$
\begin{aligned}
N^{\mathrm{Ch}}(E) & \approx \alpha \Delta \omega \Delta x_{\mathrm{PCB}}(E)\left(1-\frac{1}{v^{2} n_{\mathrm{PCB}}^{2}}\right) \\
& \approx 140\left(\frac{\Delta \omega}{7 \mathrm{eV}}\right)\left(\frac{\Delta x_{\mathrm{PCB}}(E)}{1 \mathrm{~mm}}\right) \quad(v \approx 1) .
\end{aligned}
$$

The PCB parts that can produce Cherenkov photons that can leave the PCB and be absorbed by the $\mathrm{HVeV}$ detector are determined by the PCB dimensions and the photon absorption length. Since most of the PCB's surfaces are coated with copper, Cherenkov photons generated in the middle of the PCB far away from any opening likely bounce around and are not able to escape before being absorbed again. Only those photons close to the edge or the surfaces where there is no copper covering can potentially escape and reach the detector. The relevant PCB parts are the open surface (denoted as "bare PCB" in Fig. 17 with the estimated area being $1.2 \times 1.3 \mathrm{~cm}^{2}$ ) and those parts whose distance to the edge is less than the photon absorption length. Since this absorption length is frequency dependent, the Cherenkov photons that are able to escape the PCB inherit a characteristic spectrum, shown in Fig. 18 (even if the Cherenkov emission spectrum itself [Eq. (2.4)] is rather flat for our range of frequencies). [209] Thus, the number of photons that escape the PCB per track is, on average, obtained by direct multiplication of Eq. (C2) with the spectrum shown in Fig. 17. Note that a sharp drop in the spectrum of photons that can escape the PCB is observed at frequencies $\gtrsim 8 \mathrm{eV}$, where the $\mathrm{SiO}_{2}$ becomes highly absorptive, so in what follows we drop photons with $\omega \geq 8 \mathrm{eV}$.

Having calculated the number and spectrum of photons per track that escape the PCB, we must now calculate the number of electrons that each individual track produces in the detector, which determines into which $n$-electron category $1 \leq n \leq 6$ the track-induced event falls. We assume that single photons with energies $1 \mathrm{eV} \lesssim \omega \lesssim$ $6 \mathrm{eV}$ create a single $e^{-} h^{+}$pair inside the $\mathrm{HVeV}$ detector, while photons with energies $6 \mathrm{eV} \lesssim \omega \lesssim 8 \mathrm{eV}$ can create two $e^{-} h^{+}$pairs [175]; we refer to these two energy ranges as "low" and "high," respectively. Under these assumptions, a track leads to two-electron events either by emitting one high-energy photon or by emitting two low-energy photons. The $n$-electron events with $n>2$ are obtained by multiple emission of low- or high-energy photons. If a track leads to $N_{\text {low } E}^{\mathrm{Ch}}$ low-energy and $N_{\text {high } E}^{\mathrm{Ch}}$ photons that escape the PCB, and assuming that each one of these photons has the same probability $f$ of reaching and being absorbed in the detector, the probability $P_{n}$ that a track leads to an $n$ electron event can be obtained from a binomial distribution that accounts for all possible combinations of photons of different energy that can create exactly $n e^{-} h^{+}$pairs. For example, $P_{4}$ is given by

$$
\begin{aligned}
P_{4}(E)= & \left(\begin{array}{c}
N_{\mathrm{low} E}^{\mathrm{Ch}} \\
4
\end{array}\right) f^{4}(1-f)^{N_{\text {lowE }}^{\mathrm{Ch}}+N_{\text {high }}^{\mathrm{Ch}}-4} \\
& +\left(\begin{array}{c}
N_{\mathrm{low} E}^{\mathrm{Ch}} \\
2
\end{array}\right)\left(\begin{array}{c}
N_{\text {high } E}^{\mathrm{Ch}} \\
1
\end{array}\right) f^{3}(1-f)^{N_{\mathrm{low} E}^{\mathrm{Ch}}+N_{\text {high }}^{\mathrm{Ch}}-3} \\
& +\left(\begin{array}{c}
N_{\mathrm{high} E}^{\mathrm{Ch}} \\
2
\end{array}\right) f^{2}(1-f)^{N_{\text {lowE }}^{\mathrm{Ch}}+N_{\text {high }}^{\mathrm{Ch}}-2}
\end{aligned}
$$

Note that $P_{n}(E)$ is a function of the track energy, as the number of high- and low-energy photons emitted by each track $N_{\text {low,high } E}^{\mathrm{Ch}}$ is fixed by the track energy via Eq. (C2), in conjunction with the average spectrum of photons leaving the PCB per track shown in Fig. 18. Thus, the $n-e^{-} h^{+}$event rate $R_{n}$ can be obtained by multiplying the number of tracks of a given energy passing through the areas of the PCB previously discussed, with the probability that such tracks lead to an $n$-electron event:

$$
R_{n}=\int d E R_{e, \mu}(E) P_{n}(E) .
$$

The probability $f$ is likely energy dependent and hard to quantify without a detailed simulation. For a qualitative estimate, we simply note that the predicted spectrum of $R_{n}$ for $f \approx 0.0016$ agrees well with the SuperCDMS HVeV data [71] for different bias voltages. The small value of the probability, $f \ll 1$, for each photon immediately tells us that a very large number of Cherenkov photons are created in the PCBs. Moreover, a subpercent probability is possible, because it is the combination of the geometric penalty factor for escaping the PCB, the probability of reaching the detector, and the detector efficiency. We note that the geometric penalty factor should really be included in the values of $N_{\text {low,highE }}^{\mathrm{Ch}}$, but we check that it can be absorbed into $f$ without significantly affecting the predicted values of $R_{n}$.

In summary, we show that Cherenkov radiation plausibly explains the observed events in SuperCDMS HVeV. 
However, we make several simplifying assumptions with unknown uncertainties. A dedicated simulation and more precise knowledge of the optical properties of the PCB are needed for us to draw a definitive conclusion on whether the observed events in SuperCDMS HVeV are mostly from Cherenkov.

\section{Transition radiation}

We now discuss the contribution of transition radiation in the SuperCDMS HVeV detector. As mentioned in Sec. V $\mathrm{B}$, transition radiation can be generated from charged particles passing through inner surfaces of a copper vessel, surfaces of PCBs, and any other material in the copper vessel. As long as those charged particles do not pass the detector directly, the subsequent transition radiation likely is registered as an event if they reach the detector. For our estimate, we consider only transition radiation at the inner surface of the copper vessel while neglecting other surfaces due to their smaller areas. For the dielectric function for copper, we use the data in Ref. [208] at $78 \mathrm{~K}$ for photon energies below $6.6 \mathrm{eV}$ and the data in Ref. [140] at room temperature for photon energies above $6.6 \mathrm{eV}$.

We make some simple assumptions to determine the high-energy charged particle background. First, we take the muon spectrum from Ref. [187]. Next, we assume that the ambient high-energy photon spectrum through the copper vessel looks like the SENSEI electron spectrum but scaled up by a factor of 60 , which brings the background rate in line with the measured background rate at the SuperCDMS CPD [72,183]. Similar to our estimate of transition radiation at SENSEI [see discussion around Eq. (B6)], the number of low-energy photons created in transition radiation at the SuperCDMS HVeV detector is given as

$$
\frac{d N_{\gamma}^{\mathrm{HVeV}}}{d \omega}=\sum_{i=e, \mu} \int d E R_{i}^{\mathrm{vessel}}(E) \frac{d N_{\gamma}^{\mathrm{TR}}(E)}{d \omega},
$$

where $R_{e(\mu)}^{\mathrm{vessel}}$ is the spectrum of electrons (muons) passing the inner surface of the copper vessel. In particular, $R_{e}^{\text {vessel }}=R_{\gamma}^{\text {detector }} A_{\text {vessel }} / A_{\text {detector }}\left(e^{\ell_{e}^{\text {cu }} / \ell_{\gamma}^{\text {cu }}}-1\right)$, where $R_{\gamma}^{\text {detector }}$ is the high-energy photon spectrum at the detector, $A_{\text {vessel(detector) }}$ is the surface area of the copper vessel (detector), and $\ell_{e(\gamma)}^{\mathrm{Cu}}$ is the mean range of an electron (the absorption length of a photon) inside copper. The factor $d N_{\gamma}^{\mathrm{TR}} / d \omega$ denotes the rate of transition radiation [see Eq. (3.1)], which includes both forward and backward contributions from the copper-vacuum interface. The estimated number of low-energy photons (per g-day exposure) created in transition radiation, $d N_{\gamma}^{\mathrm{HVVV}} / d \omega$, as a function of the emitted photon energy $\omega$ is shown Fig. 21.

To estimate the contribution to the observed $n$-electron events $\left(R_{n}\right)$ at SuperCDMS, we need to introduce the detection probability $f$ of each emitted photon as discussed

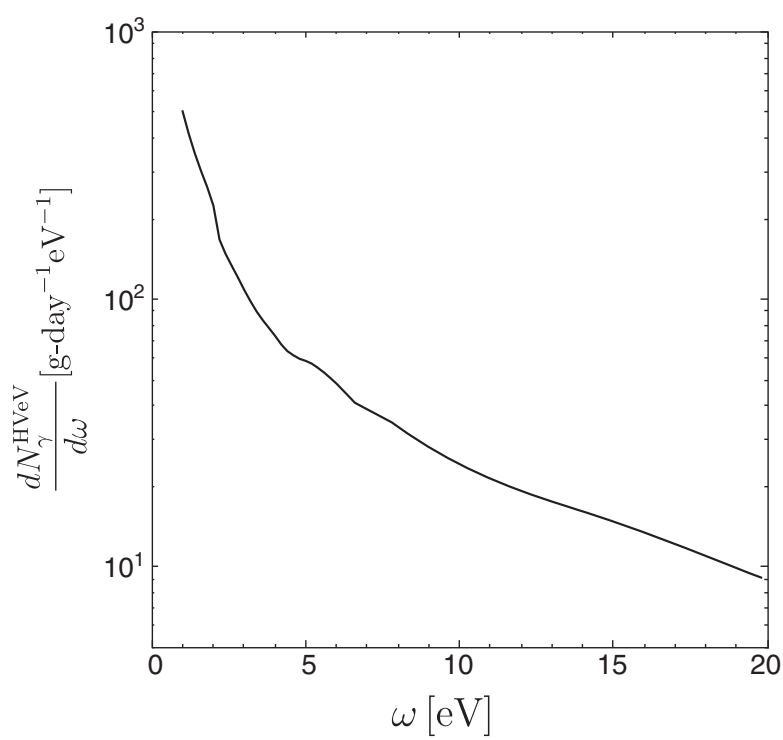

FIG. 21. The estimated number of photons (per g-day exposure) created in transition radiation from charged particles passing the interface between the copper vessel and vacuum at the SuperCDMS HVeV detector, $d N_{\gamma}^{\mathrm{HVeV}} / d \omega$, as a function of the emitted photon energy $\omega$.

in Sec. V B and above for Cherenkov radiation. Unlike the case for Cherenkov radiation, where typically tens or hundreds of Cherenkov photons are generated at (almost) the same time, typical only one photon is produced within the time window of the detector resolution. This means that only one power of $f$ is needed to obtain the rate. Moreover, since transition radiation has a spectrum of emitted photon that extends to $O(10) \mathrm{eV}$, they can create $n \geq 1 e^{-} h^{+}$pairs at the $\mathrm{Si}$ detector with the probability defined as $\eta_{n}$. Therefore, $R_{n}$ is given as

$$
R_{n}=\int d \omega \frac{d N_{\gamma}^{\mathrm{HVeV}}}{d \omega} \eta_{n}(\omega) f
$$

where $\eta_{n}(\omega)$ is take from Ref. [175] with $\sum_{n} \eta_{n}(\omega)=1$ for any $\omega$.

Since it is hard to estimate $f$ without a simulation, for illustration we simply use the inferred detection probability from our estimate of Cherenkov photons, $f \approx$ 0.0016 (see the above discussion for Cherenkov radiation). The contribution to $n$-electron events at SuperCDMS $\mathrm{HVeV}$ detector is shown in Fig. 22. This estimate suggests that transition radiation is not the dominant source of the observed events. However, we make several simplifying assumptions with unknown uncertainties, so that we hesitate to draw a definitive conclusion on the importance of transition radiation. A detailed simulation is needed to quantify the contribution from transition radiation at the SuperCDMS HVeV detector. 


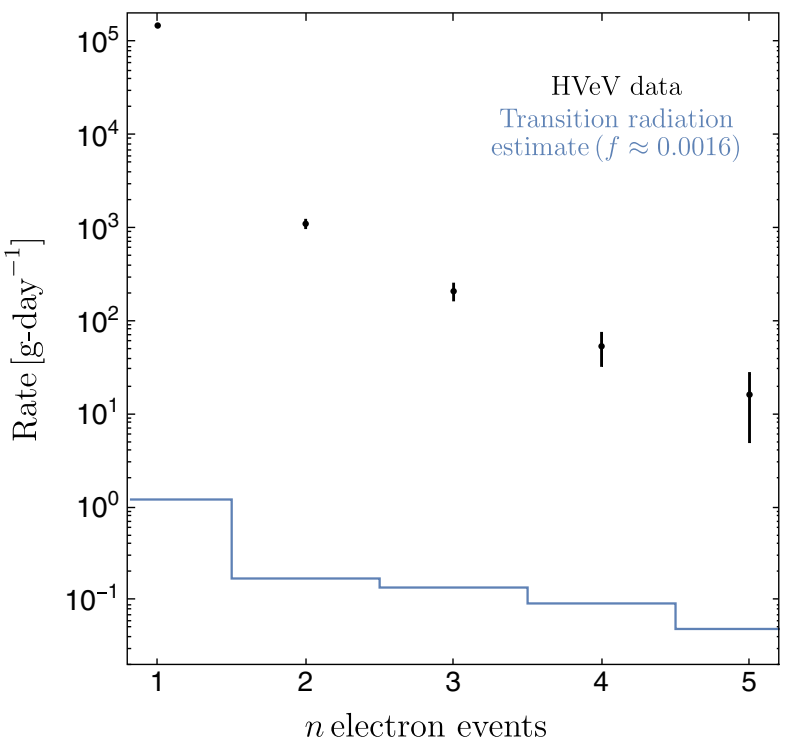

FIG. 22. The estimated $n$-electron event rate from transition radiation at SuperCDMS $\mathrm{HVeV}$ (blue line) based on a simple model with several assumptions and a photon detection probability $f \approx 1.6 \times 10^{-3}$ (see the text for details). The experimental data with $100 \mathrm{~V}$ bias voltage is shown in black with the error bars corresponding to the $3 \sigma$ statistical uncertainty [71].

\section{APPENDIX D: CHERENKOV RADIATION FOR SUPERCDMS SNOLAB DETECTOR: FURTHER DETAILS}

We provide a few additional details to our discussion in Sec. VI A on the Cherenkov background at the SuperCDMS-SNOLAB experiment. We focus on the HV detectors, which are expected to be more sensitive than the iZip detectors to the sub-keV energy depositions expected from sub-GeV dark matter.

Each HV detector inside the tower is held by 12 sensor clamps consisting of Cirlex. Each clamp has a mass of $0.17 \mathrm{~g}$; it has an irregular shape, but, approximating it as a cuboid, its dimensions are approximately $6 \mathrm{~mm} \times$ $14 \mathrm{~mm} \times 1.5 \mathrm{~mm}$. In addition, four straight and four curved detector interface boards consisting of Cirlex (of mass approximately $0.21 \mathrm{~g}$ ) sit on the edge of the copper housing near each $\mathrm{HV}$ detector and have a direct view to the $\mathrm{HV}$ detector. The shape of the detector interface boards is also irregular, but, approximating them as cuboids, their dimensions are approximately $3.8 \mathrm{~mm} \times 25.5 \mathrm{~mm} \times$ $1.5 \mathrm{~mm}$. We neglect the Cherenkov contribution from the cables connecting the HV sensors to the outside. The total mass of Cirlex that sits inside or along the side of the copper housing for each $\mathrm{HV}$ detector is, therefore, about $3.72 \mathrm{~g}$, or about $22.3 \mathrm{~g}$ for one tower consisting of six HV detectors.

In order to estimate the Cherenkov generated inside the copper housing by the Cirlex, we need to know the rate of such high-energy events that interact with the Cirlex.
The SuperCDMS Collaboration has published the expected background spectrum only up to approximately $100 \mathrm{keV}$ for the HV detectors [21], finding that detector-bulk contamination with ${ }^{32} \mathrm{Si}$ (in the $\mathrm{Si} \mathrm{HV}$ detectors) and ${ }^{3} \mathrm{H}$ (in the Ge HV detectors) dominate the background spectra at low energies. These contaminants are not present inside the Cirlex, but other radioactive impurities are present in it. These radioactive impurities are listed in Table IV of Ref. [21] and have recently been assayed more precisely $[199,200]$. Beta decays of these impurities can produce electron events with energy above $115 \mathrm{keV}$, which can create Cherenkov radiation. There is also an "ambient" environmental background that includes (i) gamma rays that Compton-scatter off electrons or pair-convert to electron-positron pairs inside the Cirlex and (ii) electrons produced in other materials that interact with the Cirlex. A simulation is required to calculate this ambient background rate and spectrum above $115 \mathrm{keV}$, although it is likely subdominant to the contribution from the radioactive impurities inside the Cirlex. In any case, our estimate of the Cherenkov event rate based on the radioactive impurities alone should be viewed as conservative.

Three radioactive impurities in Cirlex $\left({ }^{238} \mathrm{U},{ }^{232} \mathrm{Th}\right.$, and ${ }^{40} \mathrm{~K}$ ) listed in Table IV of Ref. [21] dominate the production of high-energy electrons inside the Cirlex.

(i) ${ }^{238} \mathrm{U}$ has a concentration of approximately $14 \mathrm{mBq} / \mathrm{kg}$ [199,200]. [210] The ${ }^{238} \mathrm{U}$-decay chain contains six beta decays with various energy releases $\Delta E$, namely, ${ }^{234} \mathrm{Th} \rightarrow{ }^{234 \mathrm{~m}} \mathrm{~Pa}(\Delta E \simeq 0.27 \mathrm{MeV})$, ${ }^{234 \mathrm{mPa}} \rightarrow{ }^{234} \mathrm{U} \quad(\Delta E \simeq 2.27 \mathrm{MeV}), \quad{ }^{214} \mathrm{~Pb} \rightarrow{ }^{214} \mathrm{Bi}$ $(\Delta E \simeq 1.02 \mathrm{MeV}),{ }^{214} \mathrm{Bi} \rightarrow{ }^{214} \mathrm{Po}(\Delta E \simeq 3.27 \mathrm{MeV})$, ${ }^{210} \mathrm{~Pb} \rightarrow{ }^{210} \mathrm{Bi}(\Delta E \simeq 0.06 \mathrm{MeV})$, and ${ }^{210} \mathrm{Bi} \rightarrow{ }^{210} \mathrm{Po}$ $(\Delta E \simeq 1.16 \mathrm{MeV})$. Using GEANT4, we expect about 3.8 electrons above the Cherenkov threshold of $115 \mathrm{keV}$ from the ${ }^{238} \mathrm{U}$-decay chain [199].

(ii) ${ }^{232} \mathrm{Th}$ has a concentration of approximately $4.5 \mathrm{mBq} / \mathrm{kg}[199,200]$. The ${ }^{232} \mathrm{Th}$-decay chain contains the following beta decays with various energy releases $\Delta E$, namely, ${ }^{228} \mathrm{Ra} \rightarrow{ }^{228} \mathrm{Ac}(\Delta E \simeq$ $0.05 \mathrm{MeV}), \quad{ }^{228} \mathrm{Ac} \rightarrow{ }^{228} \mathrm{Th} \quad(\Delta E \simeq 2.12 \mathrm{MeV})$, ${ }^{212} \mathrm{~Pb} \rightarrow{ }^{212} \mathrm{Bi} \quad(\Delta E \simeq 0.57 \mathrm{MeV}), \quad{ }^{212} \mathrm{Bi} \rightarrow{ }^{212} \mathrm{Po}$ $(\Delta E \simeq 2.25 \mathrm{MeV}, 64 \%)$, and ${ }^{208} \mathrm{Tl} \rightarrow{ }^{208} \mathrm{~Pb}(\Delta E \simeq$ $1.80 \mathrm{MeV}, 36 \%$ ). Using GEANT4, we expect about 2.6 electrons above the Cherenkov threshold of $115 \mathrm{keV}$ from the ${ }^{232} \mathrm{Th}$-decay chain [199].

(iii) ${ }^{40} \mathrm{~K}$ is assayed to have an upper limit on its concentration of $\lesssim 5.3 \mathrm{mBq} / \mathrm{kg}[199,200]$. We take the upper limit in our estimate of the Cherenkov event rate below. About $89 \%$ of ${ }^{40} \mathrm{~K}$ undergo a beta decay to ${ }^{40} \mathrm{Ca}$, emitting a total energy of $1.31 \mathrm{MeV}$. About $90 \%$ of the electrons released have an energy above $115 \mathrm{keV}$ [211] and produce Cherenkov photons.

Based on this information, we estimate that, in the $22.3 \mathrm{~g}$ of Cirlex present in one tower of six detectors, the total rate of 
beta decays is approximately 200 events per day or approximately 75000 events per year. The rate for producing electron events above the Cherenkov threshold of $115 \mathrm{keV}$ is approximately 130 events per day or approximately 50000 events per year.

Many of these events can be vetoed easily, although it is plausible that several Cherenkov events remain in the final dark-matter search dataset.

(i) Beta decays typically leave the daughter nucleus in an excited state and can lead to a gamma ray or $\mathrm{x}$ ray being emitted that gets subsequently absorbed in the detector. This could veto many events that produce Cherenkov radiation [199], but, of course, not all high-energy photons are observed in the detector.

(ii) A fraction of high-energy electrons produced in the beta decays leave the Cirlex and could interact in the HV detector. Nevertheless, the range of an electron with an energy of $1 \mathrm{MeV}$ in Cirlex is only about $3.3 \mathrm{~mm}$, so that many beta decays produce electrons that do not leave the Cirlex. Moreover, many electrons that leave the Cirlex simply interact with the surrounding copper without causing a signal in the HV detector. Finally, the small increase in heat from a beta decay in the Cirlex clamp is likely not detectable by the HV detector. It seems, therefore, challenging to veto many Cherenkov events using the accompanying energy deposition of the highenergy electron.

(iii) Beta decays that produce electrons with an energy well above the Cherenkov threshold of approximately $115 \mathrm{keV}$ produce a large number of photons. Such events are easy to veto, since they have a high probability of producing a signal in more than one detector. However, beta decays that produce electrons with an energy close to $115 \mathrm{keV}$ or that produce electrons that leave the Cirlex before coming to a full stop (without subsequently interacting with an HV detector) are more problematic. These produce only a few photons, which part of the time could interact only in one detector.

(iv) Some of the photons, especially those produced in the detector interface boards along the side wall of the copper housing, could leave the copper housing. This may allow at least some beta decays that produce electrons with energy well above the Cherenkov threshold to produce only a few photons that are actually observed in a single HV detector.

(v) It is possible that events produced close to the surface on the cylindrical side walls of the HV detectors can be distinguished from those that penetrate some distance into the bulk from the side walls. This would allow many events to be vetoed that produce Cherenkov photons that all hit a single detector, since they produce multiple photons, several of which are well above the Si or Ge band gap and, hence, absorbed on the side walls. However, photons that hit the top or bottom surfaces of the detector are likely harder to distinguish from those that penetrate into the bulk [94]. Even in this case, some beta decays could produce a few photons all of which are within approximately $0.1-0.2 \mathrm{eV}$ of the $\mathrm{Si}$ or (with lower probability) the Ge band gaps and have a large absorption length that allows them to penetrate into the bulk.

Finally, we assume in our discussion that the HV detectors are operated with a nonzero bias voltage. It is also possible that they operate without a bias voltage. (This would not dramatically affect their sensitivity to nuclear recoils from dark matter, although it would largely remove any sensitivity to electron recoils.) Since the design goal of the phonon energy resolution for the $\mathrm{Si}(\mathrm{Ge}) \mathrm{HV}$ detectors is about $5 \mathrm{eV}(10 \mathrm{eV}$ ) (see Table I in Ref. [21]), the detector "threshold," taken to be $7 \sigma$ above their resolution, is about $35 \mathrm{eV}(70 \mathrm{eV})$. This implies that about 35 (70) Cherenkov photons with an energy of $\mathcal{O}(\mathrm{eV})$ are needed in $\mathrm{Si}(\mathrm{Ge})$ to create a background event above the noise threshold. This is possible with the beta decays that produce electrons well above the Cherenkov threshold of $115 \mathrm{keV}$. While the Cherenkov photons from such high-energy beta decays are likely absorbed in multiple HV detectors, it is not above threshold in all of them. A detailed simulation is needed also in this case to determine whether the Cherenkov background is a concern. One interesting option might be to operate some HV detectors with a nonzero bias voltage and use these as a veto for the HV detectors that are being operated with a zero bias voltage.

[1] R. Essig, J. Mardon, and T. Volansky, Direct Detection of Sub-GeV Dark Matter, Phys. Rev. D 85, 076007 (2012).

[2] R. Essig, A. Manalaysay, J. Mardon, P. Sorensen, and T. Volansky, First Direct Detection Limits on Sub-GeV Dark Matter from Xenon10, Phys. Rev. Lett. 109, 021301 (2012).

[3] J. Angle et al. (XENON10 Collaboration), A Search for Light Dark Matter in Xenon10 Data, Phys. Rev. Lett. 107, 051301 (2011); 110, 249901(E) (2013).

[4] P. W. Graham, D. E. Kaplan, S. Rajendran, and M. T. Walters, Semiconductor Probes of Light Dark Matter, Phys. Dark Universe 1, 32 (2012).

[5] H. An, M. Pospelov, J. Pradler, and A. Ritz, Direct Detection Constraints on Dark Photon Dark Matter, Phys. Lett. B 747, 331 (2015).

[6] E. Aprile et al. (XENON100 Collaboration), First Axion Results from the Xenon100 Experiment, Phys. Rev. D 90, 062009 (2014).

[7] R. Essig, M. Fernandez-Serra, J. Mardon, A. Soto, T. Volansky, and T.-T. Yu, Direct Detection of Sub-GeV Dark Matter with Semiconductor Targets, J. High Energy Phys. 05 (2016) 046.

[8] S. K. Lee, M. Lisanti, S. Mishra-Sharma, and B. R. Safdi, Modulation Effects in Dark Matter-Electron Scattering Experiments, Phys. Rev. D 92, 083517 (2015). 
[9] Y. Hochberg, Y. Zhao, and K. M. Zurek, Superconducting Detectors for Superlight Dark Matter, Phys. Rev. Lett. 116, 011301 (2016).

[10] Y. Hochberg, M. Pyle, Y. Zhao, and K. M. Zurek, Detecting Superlight Dark Matter with Fermi-Degenerate Materials, J. High Energy Phys. 08 (2016) 057.

[11] R. Essig, J. Mardon, O. Slone, and T. Volansky, Detection of Sub-GeV Dark Matter and Solar Neutrinos via Chemical-Bond Breaking, Phys. Rev. D 95, 056011 (2017).

[12] S. Derenzo, R. Essig, A. Massari, A. Soto, and T.-T. Yu, Direct Detection of Sub-GeV Dark Matter with Scintillating Targets, Phys. Rev. D 96, 016026 (2017).

[13] A. Aguilar-Arevalo et al. (DAMIC Collaboration), First Direct-Detection Constraints on eV-Scale Hidden-Photon Dark Matter with Damic at Snolab, Phys. Rev. Lett. 118, 141803 (2017).

[14] I. M. Bloch, R. Essig, K. Tobioka, T. Volansky, and T.-T. Yu, Searching for Dark Absorption with Direct Detection Experiments, J. High Energy Phys. 06 (2017) 087.

[15] G. Cavoto, E. N. M. Cirillo, F. Cocina, J. Ferretti, and A. D. Polosa, WIMP Detection and Slow Ion Dynamics in Carbon Nanotube Arrays, Eur. Phys. J. C 76, 349 (2016).

[16] Y. Hochberg, Y. Kahn, M. Lisanti, C. G. Tully, and K. M. Zurek, Directional Detection of Dark Matter with TwoDimensional Targets, Phys. Lett. B 772, 239 (2017).

[17] Y. Hochberg, T. Lin, and K. M. Zurek, Detecting Ultralight Bosonic Dark Matter via Absorption in Superconductors, Phys. Rev. D 94, 015019 (2016).

[18] Y. Hochberg, T. Lin, and K. M. Zurek, Absorption of Light Dark Matter in Semiconductors, Phys. Rev. D 95, 023013 (2017).

[19] C. Kouvaris and J. Pradler, Probing Sub-GeV Dark Matter with Conventional Detectors, Phys. Rev. Lett. 118, 031803 (2017).

[20] E. Aprile et al. (XENON Collaboration), Low-Mass Dark Matter Search Using Ionization Signals in Xenon100, Phys. Rev. D 94, 092001 (2016); 95, 059901 (E) (2017).

[21] R. Agnese et al. (SuperCDMS Collaboration), Projected Sensitivity of the SuperCDMS SNOLAB Experiment, Phys. Rev. D 95, 082002 (2017).

[22] J. Tiffenberg, M. Sofo-Haro, A. Drlica-Wagner, R. Essig, Y. Guardincerri, S. Holland, T. Volansky, and T.-T. Yu (SENSEI Collaboration), Single-Electron and SinglePhoton Sensitivity with a Silicon Skipper CCD, Phys. Rev. Lett. 119, 131802 (2017).

[23] R. K. Romani et al., Thermal Detection of Single E-H Pairs in a Biased Silicon Crystal Detector, Appl. Phys. Lett. 112, 043501 (2018).

[24] R. Essig, T. Volansky, and T.-T. Yu, New Constraints and Prospects for Sub-GeV Dark Matter Scattering off Electrons in Xenon, Phys. Rev. D 96, 043017 (2017).

[25] R. Budnik, O. Chesnovsky, O. Slone, and T. Volansky, Direct Detection of Light Dark Matter and Solar Neutrinos via Color Center Production in Crystals, Phys. Lett. B 782, 242 (2018).

[26] P. C. Bunting, G. Gratta, T. Melia, and S. Rajendran, Magnetic Bubble Chambers and Sub-GeV Dark Matter Direct Detection, Phys. Rev. D 95, 095001 (2017).
[27] G. Cavoto, F. Luchetta, and A. D. Polosa, Sub-GeV Dark Matter Detection with Electron Recoils in Carbon Nanotubes, Phys. Lett. B 776, 338 (2018).

[28] S. Fichet, Quantum Forces from Dark Matter and Where to Find Them, Phys. Rev. Lett. 120, 131801 (2018).

[29] S. Knapen, T. Lin, M. Pyle, and K. M. Zurek, Detection of Light Dark Matter with Optical Phonons in Polar Materials, Phys. Lett. B 785, 386 (2018).

[30] Y. Hochberg, Y. Kahn, M. Lisanti, K. M. Zurek, A. G. Grushin, R. Ilan, S. M. Griffin, Z.-F. Liu, S. F. Weber, and J. B. Neaton, Detection of Sub-MeV Dark Matter with Three-Dimensional Dirac Materials, Phys. Rev. D 97, 015004 (2018).

[31] M. J. Dolan, F. Kahlhoefer, and C. McCabe, Directly Detecting Sub-GeV Dark Matter with Electrons from Nuclear Scattering, Phys. Rev. Lett. 121, 101801 (2018).

[32] M. Ibe, W. Nakano, Y. Shoji, and K. Suzuki, Migdal Effect in Dark Matter Direct Detection Experiments, J. High Energy Phys. 03 (2018) 194.

[33] G. Angloher et al. (CRESST Collaboration), Results on MeV-Scale Dark Matter from a Gram-Scale Cryogenic Calorimeter Operated above Ground, Eur. Phys. J. C 77, 637 (2017).

[34] R. Essig, M. Sholapurkar, and T.-T. Yu, Solar Neutrinos as a Signal and Background in Direct-Detection Experiments Searching for Sub-GeV Dark Matter with Electron Recoils, Phys. Rev. D 97, 095029 (2018).

[35] M. Crisler, R. Essig, J. Estrada, G. Fernandez, J. Tiffenberg, M. S. Haro, T. Volansky, and T.-T. Yu (SENSEI Collaboration), SENSEI: First Direct-Detection Constraints on Sub-GeV Dark Matter from a Surface Run, Phys. Rev. Lett. 121, 061803 (2018).

[36] R. Agnese et al. (SuperCDMS Collaboration), First Dark Matter Constraints from a SuperCDMS Single-Charge Sensitive Detector, Phys. Rev. Lett. 121, 051301 (2018); 122, 069901(E) (2019).

[37] P. Agnes et al. (DarkSide Collaboration), Constraints on Sub-GeV Dark-Matter-Electron Scattering from the DarkSide-50 Experiment, Phys. Rev. Lett. 121, 111303 (2018).

[38] M. Settimo (DAMIC Collaboration), The Damic Experiment at Snolab, arXiv:1805.10001.

[39] T. Bringmann and M. Pospelov, Novel Direct Detection Constraints on Light Dark Matter, Phys. Rev. Lett. 122, 171801 (2019).

[40] Y. Ema, F. Sala, and R. Sato, Light Dark Matter at Neutrino Experiments, Phys. Rev. Lett. 122, 181802 (2019).

[41] D. S. Akerib et al. (LUX Collaboration), Results of a Search for Sub-GeV Dark Matter Using 2013 LUX Data, Phys. Rev. Lett. 122, 131301 (2019).

[42] S. A. Hertel, A. Biekert, J. Lin, V. Velan, and D. N. McKinsey, Direct Detection of Sub-GeV Dark Matter Using a Superfluid ${ }^{4} \mathrm{He}$ Target, Phys. Rev. D 100, 092007 (2019).

[43] M. Szydagis, Y. Huang, A. C. Kamaha, C. C. Knight, C. Levy, and G. R. Rischbieter, Evidence for the Freezing of Supercooled Water by Means of Neutron Irradiation, arXiv:1807.09253 [Phys. Chem. Chem. Phys. (to be published)]. 
[44] R. Essig, J. Pérez-Ríos, H. Ramani, and O. Slone, Direct Detection of Spin-(In)dependent Nuclear Scattering of Sub-GeV Dark Matter Using Molecular Excitations, Phys. Rev. Research 1, 033105 (2019).

[45] R. Essig, J. Pradler, M. Sholapurkar, and T.-T. Yu, Relation between the Migdal Effect and Dark Matter-Electron Scattering in Isolated Atoms and Semiconductors, Phys. Rev. Lett. 124, 021801 (2020).

[46] O. Abramoff et al. (SENSEI Collaboration), SENSEI: Direct-Detection Constraints on Sub-GeV Dark Matter from a Shallow Underground Run Using a Prototype Skipper-CCD, Phys. Rev. Lett. 122, 161801 (2019).

[47] A. Aguilar-Arevalo et al. (DAMIC Collaboration), Constraints on Light Dark Matter Particles Interacting with Electrons from Damic at Snolab, Phys. Rev. Lett. 123, 181802 (2019).

[48] E. Armengaud et al. (EDELWEISS Collaboration), Searching for Low-Mass Dark Matter Particles with a Massive Ge Bolometer Operated Above-Ground, Phys. Rev. D 99, 082003 (2019).

[49] Z.Z. Liu et al. (CDEX Collaboration), Constraints on Spin-Independent Nucleus Scattering with Sub-GeV Weakly Interacting Massive Particle Dark Matter from the CDEX-1B Experiment at the China Jinping Underground Laboratory, Phys. Rev. Lett. 123, 161301 (2019).

[50] E. Aprile et al. (XENON Collaboration), Search for Light Dark Matter Interactions Enhanced by the Migdal Effect or Bremsstrahlung in Xenon1T, Phys. Rev. Lett. 123, 241803 (2019).

[51] T. Emken, R. Essig, C. Kouvaris, and M. Sholapurkar, Direct Detection of Strongly Interacting Sub-GeV Dark Matter via Electron Recoils, J. Cosmol. Astropart. Phys. 09 (2019) 070.

[52] N. F. Bell, J. B. Dent, J. L. Newstead, S. Sabharwale, and T. J. Weiler, The Migdal Effect and Photon Bremsstrahlung in Effective Field Theories of Dark Matter Direct Detection and Coherent Elastic Neutrino-Nucleus Scattering, Phys. Rev. D 101, 015012 (2020).

[53] C. V. Cappiello and J. F. Beacom, Strong New Limits on Light Dark Matter from Neutrino Experiments, Phys. Rev. D 100, 103011 (2019).

[54] T. Trickle, Z. Zhang, and K. M. Zurek, Detecting Light Dark Matter with Magnons, Phys. Rev. Lett. 124, 201801 (2020).

[55] S. M. Griffin, K. Inzani, T. Trickle, Z. Zhang, and K. M. Zurek, Multichannel Direct Detection of Light Dark Matter: Target Comparison, Phys. Rev. D 101, 055004 (2020).

[56] T. Trickle, Z. Zhang, K. M. Zurek, K. Inzani, and S. Griffin, Multi-Channel Direct Detection of Light Dark Matter: Theoretical Framework, J. High Energy Phys. 03 (2020) 036.

[57] D. Baxter, Y. Kahn, and G. Krnjaic, Electron Ionization via Dark Matter-Electron Scattering and the Migdal Effect, Phys. Rev. D 101, 076014 (2020).

[58] R. Catena, T. Emken, N. Spaldin, and W. Tarantino, Atomic Responses to General Dark Matter-Electron Interactions, Phys. Rev. Research 2, 033195 (2020).

[59] C. Blanco, J. I. Collar, Y. Kahn, and B. Lillard, Dark Matter-Electron Scattering from Aromatic Organic Targets, Phys. Rev. D 101, 056001 (2020).
[60] A. Berlin, R. T. D’Agnolo, S. A. R. Ellis, P. Schuster, and N. Toro, Direct Deflection of Particle Dark Matter, Phys. Rev. Lett. 124, 011801 (2020).

[61] A. Abdelhameed et al. (CRESST Collaboration), First Results from the CRESST-III Low-Mass Dark Matter Program, Phys. Rev. D 100, 102002 (2019).

[62] A. Abdelhameed et al. (CRESST Collaboration), Description of CRESST-III Data, arXiv:1905.07335.

[63] E. Aprile et al. (XENON Collaboration), Light Dark Matter Search with Ionization Signals in Xenon1T, Phys. Rev. Lett. 123, 251801 (2019).

[64] N. A. Kurinsky, T. C. Yu, Y. Hochberg, and B. Cabrera, Diamond Detectors for Direct Detection of Sub-GeV Dark Matter, Phys. Rev. D 99, 123005 (2019).

[65] N. Kurinsky, D. Baxter, Y. Kahn, and G. Krnjaic, Dark Matter Interpretation of Excesses in Multiple Direct Detection Experiments, Phys. Rev. D 102, 015017 (2020).

[66] A. E. Robinson and E. Michaud, Comment on "Dark Matter Interpretation of Excesses in Multiple Direct Detection Experiments, "arXiv:2002.08893.

[67] N. Kurinsky, D. Baxter, Y. Kahn, G. Krnjaic, and P. Abbamonte, Reply to Robinson and Michaud, arXiv: 2003.00101 .

[68] L. Barak et al. (SENSEI Collaboration), "SENSEI: DirectDetection Results on Sub-GeV Dark Matter from a New Skipper-CCD, Phys. Rev. Lett. 125, 171802 (2020).

[69] E. Aprile et al. (XENON Collaboration), Observation of Excess Electronic Recoil Events in XENON1T, Phys. Rev. D 102, 072004 (2020).

[70] Q. Arnaud et al. (EDELWEISS Collaboration), First Germanium-Based Constraints on Sub-MeV Dark Matter with the EDELWEISS Experiment, Phys. Rev. Lett. 125, 141301 (2020).

[71] D. Amaral et al. (SuperCDMS Collaboration), Constraints on Low-Mass, Relic Dark Matter Candidates from a Surface-Operated SuperCDMS Single-Charge Sensitive Detector, Phys. Rev. D 102, 091101 (2020).

[72] I. Alkhatib et al. (SuperCDMS Collaboration), Light Dark Matter Search with a High-Resolution Athermal Phonon Detector Operated above Ground, Phys. Rev. Lett. 127, 061801 (2021).

[73] S. M. Griffin, Y. Hochberg, K. Inzani, N. Kurinsky, T. Lin, and T. C. Yu, SiC Detectors for Sub-GeV Dark Matter, Phys. Rev. D 103, 075002 (2021).

[74] J. Alexander et al., Dark Sectors 2016 Workshop: Community Report, arXiv:1608.08632, http://lss.fnal .gov/archive/2016/conf/fermilab-conf-16-421.pdf.

[75] M. Battaglieri et al., in U.S. Cosmic Visions: New Ideas in Dark Matter College Park, MD, 2017, arXiv:1707.04591, http://lss.fnal.gov/archive/2017/conf/fermilab-conf-17-282ae-ppd-t.pdf.

[76] Report of the Workshop, Basic Research Needs for Dark Matter Small Projects New Initiatives is available online at https://science.osti.gov/-/media/hep/pdf/Reports/Dark_ Matter_New_Initiatives_rpt.pdf.

[77] E. Santos et al. (ZEPLIN-III Collaboration), Single Electron Emission in Two-Phase Xenon with Application to the Detection of Coherent Neutrino-Nucleus Scattering, J. High Energy Phys. 12 (2011) 115. 
[78] XENON100 Collaboration, Observation and applications of single-electron charge signals in the XENON100 experiment, J. Phys. G 41, 035201 (2014).

[79] P. Sorensen, Electron Train Backgrounds in Liquid Xenon Dark Matter Search Detectors Are Indeed due to Thermalization and Trapping, arXiv:1702.04805.

[80] P. Sorensen and K. Kamdin, Two Distinct Components of the Delayed Single Electron Noise in Liquid Xenon Emission Detectors, J. Instrum. 13, P02032 (2018).

[81] A. Tomás, H. Araújo, A. Bailey, A. Bayer, E. Chen, B. L. Paredes, and T. Sumner, Study and Mitigation of Spurious Electron Emission from Cathodic Wires in Noble Liquid Time Projection Chambers, Astropart. Phys. 103, 49 (2018).

[82] D. Y. Akimov et al. (RED-100 Collaboration), First Ground-Level Laboratory Test of the Two-Phase Xenon Emission Detector RED-100, J. Instrum. 15, P02020 (2020).

[83] D. S. Akerib et al. (LUX Collaboration), Investigation of Background Electron Emission in the LUX Detector, Phys. Rev. D 102, 092004 (2020).

[84] J. Àström et al., Fracture Processes Observed with a Cryogenic Detector, Phys. Lett. A 356, 262 (2006).

[85] J. Billard, E. Figueroa-Feliciano, and L. Strigari, Implication of Neutrino Backgrounds on the Reach of Next Generation Dark Matter Direct Detection Experiments, Phys. Rev. D 89, 023524 (2014).

[86] Cherenkov radiation, in particular, has been explored as a background in the context of weakly interacting massive particle detection in DarkSide [87].

[87] X. Xiang, Cherenkov Related Backgrounds in the DarkSide-50 Experiment, Ph.D. thesis, Princeton University, 2018.

[88] N. Castelló-Mor (DAMIC-M Collaboration), DAMIC-M Experiment: Thick, Silicon CCDs to Search for Light Dark Matter, Nucl. Instrum. Methods Phys. Res., Sect. A 958, 162933 (2020).

[89] Successful Department of Energy Dark Matter New Initiatives proposals: https://science.osti.gov/-/media/hep/ pdf/Awards/Dark-Matter-New-Initiatives-FY-2019-List-ofAwards.pdf.

[90] TESSERACT Collaboration, The TESSERACT Dark Matter Project, SNOWMASS LOI, http://www.snowmass 21 .org/docs/files/summaries/CF/SNOWMASS21-CF1_CF2IF1_IF8-120.pdf.

[91] One exception to this is radiative backgrounds from slow luminescence (phosphorescence), in which case the emission could occur well after a track has passed. This "afterglow" is challenging to veto and could be important for some dark-matter searches (see discussion in, e.g., Refs. [12,92]). A comprehensive study of luminescence for all materials typically present in dark-matter detectors is still lacking.

[92] S. Derenzo, E. Bourret, S. Hanrahan, and G. Bizarri, Cryogenic Scintillation Properties of n-Type GaAs for the Direct Detection of $\mathrm{MeV} / \mathrm{c}^{2}$ Dark Matter, J. Appl. Phys. 123, 114501 (2018).

[93] See talks at EXCESS workshop, 2021, https://indico.cern .ch/event/1013203/.

[94] M. Pyle (private communication).
[95] A. Aguilar-Arevalo et al. (CONNIE Collaboration), Exploring Low-Energy Neutrino Physics with the Coherent Neutrino Nucleus Interaction Experiment, Phys. Rev. D 100, 092005 (2019).

[96] G. Fernandez-Moroni, P. A. N. Machado, I. MartinezSoler, Y. F. Perez-Gonzalez, D. Rodrigues, and S. Rosauro-Alcaraz, The Physics Potential of a Reactor Neutrino Experiment with Skipper CCDs: Measuring the Weak Mixing Angle, J. High Energy Phys. 03 (2021) 186.

[97] A. Vepsäläinen et al., Impact of Ionizing Radiation on Superconducting Qubit Coherence, Nature (London) 584, 551 (2020).

[98] L. Cardani et al., Reducing the Impact of Radioactivity on Quantum Circuits in a Deep-Underground Facility, Nat. Commun. 12, 2733 (2021).

[99] C. D. Wilen et al., Correlated Charge Noise and Relaxation Errors in Superconducting Qubits, Nature (London) 594, 369 (2021).

[100] M. Mcewen et al., Resolving Catastrophic Error Bursts from Cosmic Rays in Large Arrays of Superconducting Qubits, arXiv:2104.05219.

[101] P. Budini, Energy Loss and Čerenkov Radiation of a Relativistic Ionizing Particle, Phys. Rev. 89, 1147 (1953).

[102] R. M. Sternheimer, The Energy Loss of a Fast Charged Particle by Čerenkov Radiation, Phys. Rev. 91, 256 (1953).

[103] The power counting of the Cherenkov rate requires some explanation. For Cherenkov radiation to happen, the dielectric function needs to be larger than one. Given that $\epsilon(\omega)$ is set by electromagnetic interactions, it would seem that $1-\epsilon(\omega) \propto \alpha$, which would make Cherenkov radiation an $\mathcal{O}\left(\alpha^{2}\right)$ effect. However, it turns out that in dielectrics $\epsilon(\omega)$ deviates from one at zeroth order in $\alpha$. The reason is that, while at small $\alpha$ photons couple less strongly to the electrons in the material, at the same time electrons become less bound to the atoms, making the material more polarizable. These competing effects cancel out, leading to $1-$ $\epsilon(\omega) \sim \mathcal{O}(1)$ [104]. Thus, Cherenkov radiation is, in fact, an $\mathcal{O}(\alpha)$ process in dielectric materials.

[104] J. D. Jackson, Classical Electrodynamics (Wiley, New York, 1998).

[105] Equations (2.3)-(2.5) do not take into account corrections due to angular aberration, which are of the order of $\mathcal{O}[\operatorname{Im} \epsilon(\omega) / \operatorname{Re} \epsilon(\omega)][106]$. For all practical purposes, in this work such corrections can be safely neglected, as when estimating backgrounds at dark-matter detectors we are interested only in Cherenkov radiation in the transparent or semitransparent regimes where $\operatorname{Re} \epsilon(\omega) \gg \operatorname{Im} \epsilon(\omega)$.

[106] V. Grichine, On the Energy-Angle Distribution of Cherenkov Radiation in an Absorbing Medium, Nucl. Instrum. Methods Phys. Res., Sect. A 482, 629 (2002).

[107] S. M. Neamtan, The Čerenkov Effect and the Dielectric Constant, Phys. Rev. 92, 1362 (1953).

[108] Y. Varshni, Temperature Dependence of the Energy Gap in Semiconductors, Physica (Utrecht) 34, 149 (1967).

[109] R. H. French, Electronic Band Structure of Al2O3, with Comparison to AlON and AIN, J. Am. Ceram. Soc. 73, 477 (1990).

[110] T. DiStefano and D. Eastman, The Band Edge of Amorphous SiO2 by Photoinjection and Photoconductivity Measurements, Solid State Commun. 9, 2259 (1971). 
[111] R. Agnese et al. (SuperCDMS Collaboration), Search for Low-Mass Weakly Interacting Massive Particles with SuperCDMS, Phys. Rev. Lett. 112, 241302 (2014).

[112] J. Barreto et al. (DAMIC Collaboration), Direct Search for Low Mass Dark Matter Particles with CCDs, Phys. Lett. B 711, 264 (2012).

[113] P. Y. Yu and M. Cardona, Fundamentals of Semiconductors: Physics and Materials Properties (Springer, Berlin, Heidelberg, 2005).

[114] E. D. Palik, Handbook of Optical Constants of Solids (Elsevier, 1985), 10.1016/C2009-0-20920-2.

[115] F. A. Johnson, Lattice Absorption Bands in Silicon, Proc. Phys. Soc. 73, 265 (1959).

[116] R. J. Collins and H. Y. Fan, Infrared Lattice Absorption Bands in Germanium, Silicon, and Diamond, Phys. Rev. 93, 674 (1954).

[117] W. G. Spitzer and D. A. Kleinman, Infrared Lattice Bands of Quartz, Phys. Rev. 121, 1324 (1961).

[118] A. S. Barker, Infrared Lattice Vibrations and Dielectric Dispersion in Corundum, Phys. Rev. 132, 1474 (1963).

[119] T. Tomiki, Y. Ganaha, T. Futemma, T. Shikenbaru, Y. Aiura, M. Yuri, S. Sato, H. Fukutani, H. Kato, T. Miyahara, J. Tamashiro, and A. Yonesu, Anisotropic Optical Spectra of Al2O3 Single Crystals in the Vacuum Ultraviolet Region. II. Spectra of Optical Constants, J. Phys. Soc. Jpn. 62, 1372 (1993).

[120] J. C. Phillips, Dielectric Definition of Electronegativity, Phys. Rev. Lett. 20, 550 (1968).

[121] N. W. Ashcroft, N. D. Mermin, and R. Smoluchowski, Solid State Physics, Phys. Today 30, No. 1, 61 (1977).

[122] M. M. J. Berger, J. Coursey, and J. Chang, Stopping-Power \& Range Tables for Electrons, Protons, and Helium Ions: NIST Standard Reference Database 124, https://physics .nist.gov/PhysRefData/Star/Text/method.html.

[123] M. Tanabashi et al. (Particle Data Group), Review of Particle Physics, Phys. Rev. D 98, 030001 (2018).

[124] C. Tsai, Interband and Intraband Absorption Coefficients of Silicon: Theoretical Frameworks and Formulations, IEEE J. Sel. Top. Quantum Electron. 26, 1 (2020).

[125] G. Macfarlane, T. McLean, J. Quarrington, and V. Roberts, Fine Structure in the Absorption-Edge Spectrum of Si, Phys. Rev. 111, 1245 (1958).

[126] G. Macfarlane, T. McLean, J. Quarrington, and V. Roberts, Fine Structure in the Absorption-Edge Spectrum of Ge, Phys. Rev. 108, 1377 (1957).

[127] M. Sturge, Optical Absorption of Gallium Arsenide between 0.6 and $2.75 \mathrm{eV}$, Phys. Rev. 127, 768 (1962).

[128] R. H. French, Electronic Band Structure of Al2O3, with Comparison to AlON and AIN, J. Am. Ceram. Soc. 73, 477 (1990).

[129] I. Godmanis, A. N. Trukhin, and K. Hübner, ExcitonPhonon Interaction in Crystalline and Vitreous SiO2, Phys. Status Solidi (b) 116, 279 (1983).

[130] W. Dash and R. Newman, Intrinsic Optical Absorption in Single-Crystal Germanium and Silicon at $77 \mathrm{~K}$ and $300 \mathrm{~K}$, Phys. Rev. 99, 1151 (1955).

[131] M. L. Ter-Mikaelian, High Energy Electromagnetic Processes in Condensed Media, ISBN: 9780471851905.
[132] V. L. Ginzburg and V. Tsytovich, Several Problems of the Theory of Transition Radiation and Transition Scattering, Phys. Rep. 49, 1 (1979).

[133] V.L. Ginzburg, Theoretical Physics and Astrophysics (Elsevier, New York, 2013).

[134] V. Zrelov and J. Ruzicka, Some Peculiarities of the Optical Transition Radiation from High-Energy Particles at Inclined Incidence (III), Nucl. Instrum. Methods 151, 395 (1978).

[135] V.P. Zrelov and J. Ružička, Hybrid Radiation and its Properties IV, Nucl. Instrum. Methods 160, 327 (1979).

[136] In Ref. [135], Cherenkov-transition radiation is called hybrid radiation.

[137] L. L. DeRaad, Jr., W.-Y. Tsai, and T. Erber, Interference between Transition and Čerenkov Radiation, Phys. Rev. D 18, 2152 (1978).

[138] This simplified scenario where no conventional Cherenkov radiation is possible is the most widely discussed in the literature; see, e.g., Refs. [104,132,139].

[139] L. D. Landau, J. Bell, M. Kearsley, L. Pitaevskii, E. Lifshitz, and J. Sykes, Electrodynamics of Continuous Media (Elsevier, New York, 2013), Vol. 8.

[140] W. S. M. Werner, K. Glantschnig, and C. Ambrosch-Draxl, Optical Constants and Inelastic Electron-Scattering Data for 17 Elemental Metals, J. Phys. Chem. Ref. Data 38, 1013 (2009).

[141] W. Shockley, Problems Related to p-n Junctions in Silicon, Czech. J. Phys. 11, 81 (1961).

[142] R. Alig and S. Bloom, Electron-Hole-Pair Creation Energies in Semiconductors, Phys. Rev. Lett. 35, 1522 (1975).

[143] D. Rodrigues et al., Absolute Measurement of the Fano Factor Using a Skipper-CCD, Nucl. Instrum. Methods Phys. Res., Sect. A 1010, 165511 (2021).

[144] R. Ryan, Precision Measurements of the Ionization Energy and Its Temperature Variation in High Purity Silicon Radiation Detectors, IEEE Trans. Nucl. Sci. 20, 473 (1973).

[145] G. Bertuccio and D. Maiocchi, Electron-Hole Pair Generation Energy in Gallium Arsenide by $x$ and $\gamma$ Photons, J. Appl. Phys. 92, 1248 (2002).

[146] C. Klein, Simple Explanation of the Electron-Hole Pair Creation Energy Puzzle in Germanium, Phys. Lett. A 24, 513 (1967).

[147] C. Hu, Modern Semiconductor Devices for Integrated Circuits (2009), ISBN: 9788131730249.

[148] H. Schlangenotto, H. Maeder, and W. Gerlach, Temperature Dependence of the Radiative Recombination Coefficient in Silicon, Phys. Status Solidi (a) 21, 357 (1974).

[149] R. N. Hall, Recombination Processes in Semiconductors, Proc. Inst. Electr. Eng., Part B 106, 923 (1959).

[150] C. Nuese, H. Kressel, and I. Ladany, Future for LEDs, IEEE Spectrum 9, 28 (1972).

[151] L. Huldt, Band-to-Band Auger Recombination in Indirect Gap Semiconductors, Phys. Status Solidi (a) 8, 173 (1971).

[152] J. Dziewior and W. Schmid, Auger Coefficients for Highly Doped and Highly Excited Silicon, Appl. Phys. Lett. 31, 346 (1977).

[153] D. Steiauf, E. Kioupakis, and C. G. Van de Walle, Auger Recombination in GaAs from First Principles, ACS Photonics 1, 643 (2014). 
[154] M. Ruff, M. Fick, R. Lindner, U. Rössler, and R. Helbig, The Spectral Distribution of the Intrinsic Radiative Recombination in Silicon, J. Appl. Phys. 74, 267 (1993).

[155] M. A. Green, Silicon Solar Cells: Advanced Principles and Practice (Bridge, Sydney, 1995).

[156] R. Corkish, D. S.-P. Chan, and M. A. Green, Excitons in Silicon Diodes and Solar Cells: A Three-Particle Theory, J. Appl. Phys. 79, 195 (1996).

[157] Unless specified otherwise, we work in natural units. In SI units, a factor of the Boltzmann constant $k_{B}$ needs to be introduced on the right-hand side of Eq. (4.5).

[158] M. Melehy, Diffusion-Mobility Generalized Relationship and Average Kinetic Energy of Electrons in Solid Crystals, Proc. IEEE 53, 536 (1965).

[159] J. M. Dorkel and Ph. Leturcq, Carrier Mobilities in Silicon Semi-Empirically Related to Temperature, Doping and Injection Level, Solid-State Electron. 24, 821 (1981).

[160] In the literature, usually the product $\bar{n}_{e} \bar{n}_{h}$ is replaced by the intrinsic carrier density $n_{i}^{2}$ (see, e.g., Ref. [149]), as both quantities are always equal; see Appendix A.

[161] R. Brüggemann, M. Xu, J. Alvarez, M. Boutchich, and J.-P. Kleider, Radiative Recombination Coefficient in Crystalline Silicon at Low Temperatures $<77 \mathrm{~K}$ by Combined Photoluminescence Measurements, Energy Procedia 124, 10 (2017).

[162] R. J. Van Overstraeten and R. P. Mertens, Heavy Doping Effects in Silicon, Solid-State Electron. 30, 1077 (1987).

[163] W. Shockley and W. T. Read, Statistics of the Recombinations of Holes and Electrons, Phys. Rev. 87, 835 (1952).

[164] K. K. N. Simon and M. Sze, Physics of Semiconductor Devices, 3rd ed. (Wiley-Interscience, New York, 2006).

[165] M. S. Tyagi and R. Van Overstraeten, Minority Carrier Recombination in Heavily-Doped Silicon, Solid-State Electron. 26, 577 (1983).

[166] J. I. Pankove, Optical Processes in Semiconductors (Courier, Rockland, ME, 1975).

[167] P. T. Landsberg, Recombination in Semiconductors (Cambridge University Press, Cambridge, England, 2003).

[168] C. Hu and W. G. Oldham, Carrier Recombination through Donors/Acceptors in Heavily Doped Silicon, Appl. Phys. Lett. 35, 636 (1979).

[169] S. Wu, Z. Pan, R. Chen, and X. Liu, Long Afterglow Phosphorescent Materials (Springer, New York, 2017).

[170] We thank Steve Holland for referring to us this value.

[171] As we see, the SENSEI data provide important clues as to the origin of the single-electron events. We are grateful to several members of the SENSEI Collaboration for numerous useful discussions of the characteristics of the data and the details of the detector setup, including Mariano Cababie, Juan Estrada, Yonatan Ben Gal, Daniel Gift, Sravan Munagavalasa, Aman Singal, Javier Tiffenberg, Sho Uemura, and Tomer Volansky.

[172] We note that bremsstrahlung contributes negligibly to the rate.

[173] K. Rajkanan, R. Singh, and J. Shewchun, Absorption Coefficient of Silicon for Solar Cell Calculations, SolidState Electron. 22, 793 (1979).

[174] N. S. Allen, J. P. Binkley, B. J. Parsons, G. O. Phillips, and N. H. Tennent, Spectroscopic Properties and Photosensitivity of Epoxy Resins, Polymer photochemistry 2, 97 (1982).
[175] K. Ramanathan and N. Kurinsky, Ionization Yield in Silicon for eV-Scale Electron-Recoil Processes, Phys. Rev. D 102, 063026 (2020).

[176] Transition radiation was recently suggested to cause some of the excess events in SENSEI [177]. We believe, however, that it is subdominant to Cherenkov radiation and recombination photons.

[177] A. E. Robinson, Electrovolt-Scale Backgrounds from Surfaces, arXiv:2010.11043.

[178] P. Du, D. Egana-Ugrinovic, R. Essig, and M. Sholapurkar, On the Origin of Single-Electron Events in Skipper-CCDs and the SENSEI Sub-GeV Dark Matter Direct Detection Experiment (to be published).

[179] B. Neganov and V. TrofimovColorimetric Method Measuring Ionizing Radiation, Otkryt. Izobret. 146, 215 (1985).

[180] P. Luke, Voltage-Assisted Calorimetric Ionization Detector, J. Appl. Phys. 64, 6858 (1988).

[181] We expect an analogous discussion of radiative backgrounds to be relevant also for the detector and data described in Ref. [36] (see also Refs. [23,182]. The precise detector setup differs, however, from the one in Ref. [71] and, hence, would require a dedicated discussion and analysis that is beyond the scope of this paper.

[182] Z. Hong et al., Single Electron-Hole Pair Sensitive Silicon Detector with Surface Event Discrimination, Nucl. Instrum. Methods Phys. Res., Sect. A 963, 163757 (2020).

[183] N. Kurinsky (private communication).

[184] We thank Noah Kurinsky and Matt Pyle for very useful discussions of the HVeV detector and data. When discussing with them, it became clear that the SuperCDMS $\mathrm{HVeV}$ Collaboration had already been studying the hypothesis of photon production within the PCB holders as the source of the events containing $n \geq 2 e^{-} h^{+}$pairs. However, to our knowledge, no exact microphysical mechanism was identified by them. We believe that we have now identified several plausible mechanisms.

[185] See https://gitlab.cern.ch/geant4/geant4/blob/4ec577e5c49e900713a6c89b59a8b3e8a4b71a52/examples/advanced/ composite_calorimeter/dataglobal/material.cms.

[186] H. Durmus, H. Safak, H.Z. Akbas, and G. Ahmetli, Optical Properties of Modified Epoxy Resin with Various Oxime Derivatives in the UV-Vis Spectral Region, J. Appl. Polym. Sci. 120, 1490 (2011).

[187] L. Bogdanova, M. Gavrilov, V. Kornoukhov, and A. Starostin, Cosmic Muon Flux at Shallow Depths Underground, Phys. At. Nucl. 69, 1293 (2006).

[188] One differential-rate unit is defined as 1 event $/ \mathrm{kg} / \mathrm{day} / \mathrm{keV}$.

[189] Q. Arnaud, J. Billard, J. Gascon, and A. Juillard (private communication).

[190] S. Scorza (EDELWEISS Collaboration), Background Investigation in EDELWEISS-III, AIP Conf. Proc. 1672, 100002 (2015).

[191] M. Ghamnia, C. Jardin, and M. Bouslama, Luminescent Centres $f$ and $\mathrm{f}+$ in $\alpha$-Alumina Detected by Cathodoluminescence Technique, J. Electron Spectrosc. Relat. Phenom. 133, 55 (2003).

[192] M. Yang, R. French, and E. Tokarsky, Optical Properties of Teflon ${ }^{\circledR}$ AF Amorphous Fluoropolymers, J. Microlithogr., Microfabr., Microsyst. 7, 033010 (2008). 
[193] M. Baryakhtar, J. Huang, and R. Lasenby, Axion and Hidden Photon Dark Matter Detection with Multilayer Optical Haloscopes, Phys. Rev. D 98, 035006 (2018).

[194] We thank Masha Baryakhtar, Junwu Huang, Robert Lasenby, and Sae-Woo Nam for detailed information on the detector setup.

[195] A. Arvanitaki, S. Dimopoulos, and K. Van Tilburg, Resonant Absorption of Bosonic Dark Matter in Molecules, Phys. Rev. X 8, 041001 (2018).

[196] http://www.cirlex.com.

[197] E. T. Arakawa, M. W. Williams, J. C. Ashley, and L. R. Painter, The Optical Properties of Kapton: Measurement and Applications, J. Appl. Phys. 52, 3579 (1981).

[198] B. Ahmed, S. Raghuvanshi, Siddhartha, A. Srivastava, J. Krishna, and M. Wahab, Optical and Structural Study of Aromatic Polymers Irradiated by Gamma Radiation, Indian J. Pure Appl. Phys. 50, 892 (2012).

[199] B. Loer (private communication).

[200] SuperCDMS Collaboration, Radioactivity Measurements for the SuperCDMS SNOLAB Experiment (to be published).

[201] A. E. Robinson, The Coherent Photon Scattering Background in Sub-GeV/c Direct Dark Matter Searches, Phys. Rev. D 95, 021301(R) (2017); 95, 069907(E) (2017).

[202] N. Coron, G. Dambier, E. Leblanc, J. Leblanc, P. de Marcillac, and J.P. Moalic, Scintillating and Particle Discrimination Properties of Selected Crystals for LowTemperature Bolometers: From LiF to BGO, Nucl. Instrum. Methods Phys. Res., Sect. A 520, 159 (2004).

[203] S. Agostinelli et al. (GEANT4 Collaboration), GEANT4-A Simulation Toolkit, Nucl. Instrum. Methods Phys. Res., Sect. A 506, 250 (2003).
[204] A. E. Chavarria (Secondary-ion mass spectrometry (SIMS) on the back side of unthinned LBNL CCDs performed for the DAMIC Collaboration) (private communication).

[205] M. A. Green, Silicon Solar Cells: Advanced Principles \& Practice (Centre for Photovoltaic Devices and Systems, Sydney, Australia, 1995).

[206] C.-Y. Tsai, Interband and Intraband Absorption Coefficients of Silicon: Theoretical Frameworks and Formulations, IEEE J. Sel. Top. Quantum Electron. 26, 1 (2019).

[207] We neglect, e.g., the CCD-epoxy, CCD-vacuum, pitchadapter-vacuum, and pitch-adapter-epoxy interfaces (see the SENSEI detector schematic in Fig. 14), as well as various layers on the front and back sides of the CCD.

[208] P. B. Johnson and R. W. Christy, Optical Constants of Copper and Nickel as a Function of Temperature, Phys. Rev. B 11, 1315 (1975).

[209] To calculate the number of photons that escape the PCB, we make the simplifying assumption that, upon emission, photons take the shortest path toward the edge of the PCB and do not internally reflect. As a consequence, on top of our estimates, a geometric penalty factor must be included to account for photons that take longer paths toward the edges of the PCB or that internally reflect [see discussion below Eq. (C4)].

[210] All quoted concentrations assume that the entire decay chain is in secular equilibrium [199].

[211] X. Mougeot, Betashape: A New Code for Improved Analytical Calculations of Beta Spectra, EPJ Web Conf. 146, 12015 (2017). 